\title{
NEW AND OLD RESULTS ON SPHERICAL VARIETIES VIA MODULI THEORY
}

\author{
ROMAN AVDEEV AND STÉPHANIE CUPIT-FOUTOU
}

\begin{abstract}
Given a connected reductive algebraic group $G$ and a finitely generated monoid $\Gamma$ of dominant weights of $G$, in 2005 Alexeev and Brion constructed a moduli scheme $\mathrm{M}_{\Gamma}$ for multiplicity-free affine $G$-varieties with weight monoid $\Gamma$. This scheme is equipped with an action of an 'adjoint torus' $T_{\text {ad }}$ and has a distinguished $T_{\text {ad }}$-fixed point $X_{0}$. In this paper, we obtain a complete description of the $T_{\text {ad }}$-module structure in the tangent space of $\mathrm{M}_{\Gamma}$ at $X_{0}$ for the case where $\Gamma$ is saturated. Using this description, we prove that the root monoid of any affine spherical $G$-variety is free. As another application, we obtain new proofs of uniqueness results for affine spherical varieties and spherical homogeneous spaces first proved by Losev in 2009. Furthermore, we obtain a new proof of Alexeev and Brion's finiteness result for multiplicity-free affine $G$-varieties with a prescribed weight monoid. At last, we prove that for saturated $\Gamma$ all the irreducible components of $\mathrm{M}_{\Gamma}$, equipped with their reduced subscheme structure, are affine spaces.
\end{abstract}

\section{INTRODUCTION}

All objects considered in this paper are defined over an algebraically closed field $\mathbb{k}$ of characteristic 0 .

Let $G$ be a connected reductive algebraic group. A $G$-variety (that is, an algebraic variety equipped with a regular action of $G$ ) is called spherical if it is normal and contains a dense orbit for the induced action of a Borel subgroup $B \subset G$. Famous examples of spherical varieties are toric varieties, flag varieties, and symmetric varieties. Due to a combination of numerous works and methods, the structure theory of spherical varieties is now well understood and has recently led to a full classification of these objects; see [Ti11, Chapter 5] for a review.

In this paper we obtain new results and also recover a number of already known facts on spherical varieties via one single approach - that of moduli theory, which does not involve any classification results in the theory of spherical varieties. Specifically, we are concerned with the moduli theory developed by Alexeev and Brion in AB05] for affine spherical $G$-varieties and more generally for multiplicity-free affine $G$-varieties.

An affine $G$-variety $X$ is said to be multiplicity-free if $X$ is irreducible and the algebra $\mathbb{k}[X]$ of regular functions on $X$, regarded as a $G$-module, contains every simple $G$-module with multiplicity at most 1. By a theorem of Vinberg and Kimelfeld [ViK78], an irreducible affine $G$-variety is multiplicity-free if and only if it possesses a dense $B$-orbit. In particular, affine spherical $G$-varieties are characterized as normal multiplicity-free affine $G$-varieties.

Given a multiplicity-free affine $G$-variety $X$, the $G$-module structure of $\mathbb{k}[X]$ is encoded in the weight monoid $\Gamma_{X}$ of $X$, consisting of all dominant weights $\lambda$ of $G$ for which $\mathbb{k}[X]$

2010 Mathematics Subject Classification. 14M27, 14D22, 20 G05.

Key words and phrases. Algebraic group, multiplicity-free variety, spherical variety, moduli scheme. 
contains a simple $G$-submodule $\mathbb{k}[X]_{\lambda}$ with highest weight $\lambda$. This monoid is known to be finitely generated. Besides, $X$ is normal if and only if $\Gamma_{X}$ is saturated, that is, $\Gamma_{X}$ is the intersection of a lattice with a cone.

One more invariant of a multiplicity-free affine $G$-variety $X$ is its root monoid $\Xi_{X}$, which arises from the ring structure of $\mathbb{k}[X]$. By definition, $\Xi_{X}$ is generated by all expressions $\lambda+\mu-\nu$ with $\lambda, \mu, \nu \in \Gamma_{X}$ such that the linear span of $\mathbb{k}[X]_{\lambda} \cdot \mathbb{k}[X]_{\mu}$ contains $\mathbb{k}[X]_{\nu}$. Let $\Xi_{X}^{\text {sat }}$ denote the saturation of $\Xi_{X}$, that is, the intersection of the lattice generated by $\Xi_{X}$ with the cone spanned by $\Xi_{X}$. An important property of the root monoid was discovered by Knop in [Kn96], who proved that the monoid $\Xi_{X}^{\text {sat }}$ is free.

In [AB05, Alexeev and Brion constructed and studied a moduli scheme $\mathrm{M}_{\Gamma}$ for multiplicity-free affine $G$-varieties with prescribed weight monoid $\Gamma$. This scheme is affine and of finite type; it is equipped with an action of an adjoint torus $T_{\mathrm{ad}}$ (the quotient of a maximal torus of $G$ by the center of $G$ ) in such a way that the $T_{\text {ad }}$-orbits of $\mathrm{M}_{\Gamma}$ bijectively correspond to the $G$-isomorphism classes of multiplicity-free affine $G$-varieties with weight monoid $\Gamma$. Various examples of moduli schemes $\mathrm{M}_{\Gamma}$ were further studied under different assumptions on the monoid $\Gamma$. The case of monoids generated by a single element was worked out in [Ja07]; the paper [BCF08] dealt with free monoids that are $G$-saturated (the latter means that the monoid consists of all dominant weights of $G$ lying in a fixed lattice); several other special instances of free monoids were studied in [CF09, PVS12, PVS16. In all these cases, $\mathrm{M}_{\Gamma}$ was shown to be an affine space (as a scheme). Finally, in [BVS16] it was proved that for an arbitrary free monoid $\Gamma$ all the irreducible components of $\mathrm{M}_{\Gamma}$, equipped with their reduced subscheme structure, are affine spaces.

Given an arbitrary finitely generated monoid $\Gamma$ of dominant weights of $G$, there always exists a multiplicity-free affine $G$-variety $X_{0}=X_{0}(\Gamma)$ with weight monoid $\Gamma$ such that the linear span of $\mathbb{k}\left[X_{0}\right]_{\lambda} \cdot \mathbb{k}\left[X_{0}\right]_{\mu}$ coincides with $\mathbb{k}\left[X_{0}\right]_{\lambda+\mu}$ for all $\lambda, \mu \in \Gamma$. Such varieties were first considered and studied by Vinberg and Popov in ViP72. It is known from AB05 that the $T_{\text {ad }}$-orbit in $\mathrm{M}_{\Gamma}$ corresponding to $X_{0}$ is just a $T_{\text {ad }}$-fixed closed point (still denoted by $X_{0}$ ), hence the tangent space $T_{X_{0}} \mathrm{M}_{\Gamma}$ of $\mathrm{M}_{\Gamma}$ at $X_{0}$ is naturally equipped with the structure of a $T_{\text {ad }}$-module.

One of the main achievements of this paper is a complete description of the $T_{\mathrm{ad}}$-module structure of $T_{X_{0}} \mathrm{M}_{\Gamma}$ purely in terms of $\Gamma$ in the case where $\Gamma$ is saturated (see Theorem 3.1). In particular, we show that $T_{X_{0}} \mathrm{M}_{\Gamma}$ is a multiplicity-free $T_{\text {ad }}$-module whose weights, up to a sign, belong to a certain finite set $\bar{\Sigma}(G)$ depending only on $G$. The set $\bar{\Sigma}(G)$ turns out to be a subset of the set of spherical roots of $G$ that is well known in the theory of spherical varieties.

As a first application of our description of $T_{X_{0}} \mathrm{M}_{\Gamma}$, we show that, given an arbitrary affine spherical $G$-variety $X$, every indecomposable element of the monoid $\Xi_{X}$ is primitive in the lattice $\mathbb{Z} \Xi_{X}$ (see Proposition $4.10(\bar{b})$ ). Combining this with the above-mentioned result of Knop on the freeness of $\Xi_{X}^{\text {sat }}$, we derive that the monoid $\Xi_{X}$ itself is free (see Theorem 4.11).

As a second application, we obtain a new proof of the following uniqueness result for affine spherical $G$-varieties: up to a $G$-isomorphism, every affine spherical $G$-variety $X$ is uniquely determined by the pair $\left(\Gamma_{X}, \Sigma_{X}\right)$, where $\Sigma_{X}$ is the set of spherical roots of $X$, that is, primitive elements of the lattice $\mathbb{Z} \Gamma_{X}$ lying on extremal rays of the cone spanned by $\Xi_{X}$ (see Corollary 4.16). This fact was first proved by Losev in [Lo09b]. It is worth noticing 
that the above-mentioned uniqueness result easily extends to arbitrary multiplicity-free affine $G$-varieties (see Corollary 4.22).

As a third application, we derive a new proof of a rule that enables one to determine the set $\bar{\Sigma}_{X}$ of free generators of the monoid $\Xi_{X}^{\text {sat }}$ of an affine spherical $G$-variety $X$ starting from the set $\Sigma_{X}$ of spherical roots (see Theorem 4.19). This rule was first obtained by Losev in [Lo09a]. (In fact, Losev's result deals with a much more general situation.)

We point out that in all the three above-mentioned applications our proofs easily reduce to checking certain combinatorial properties of the set of weights of the $T_{\text {ad }}$-module $T_{X_{0}} \mathrm{M}_{\Gamma}$.

Using elementary additional material on spherical varieties, from the uniqueness result for affine spherical $G$-varieties we derive the uniqueness property for spherical homogeneous spaces first obtained by Losev in [Lo09a]; see our Theorem 4.24 for a precise statement.

We note that Losev's proofs of the above-mentioned uniqueness results for affine spherical varieties and spherical homogeneous spaces use Lie-theoretical methods; the already known classification of affine spherical homogeneous spaces comes into play in his approach. It is also worth mentioning that one more independent proof of the uniqueness property for spherical homogeneous spaces follows from a combination of Luna's paper [Lu01] and Cupit-Foutou's one [CF09], the latter dealing with more complicated aspects of moduli theory of affine spherical varieties than in this paper.

Making use of the uniqueness property for affine spherical $G$-varieties, we recover the following result first obtained by Alexeev and Brion in [AB05]: there are only finitely many $G$-isomorphism classes of multiplicity-free affine $G$-varieties with prescribed weight monoid $\Gamma$ (see Corollary 4.23); equivalently, $\mathrm{M}_{\Gamma}$ contains only finitely many $T_{\mathrm{ad}}$-orbits. The initial proof of this fact given in $\mathrm{AB} 05$ ] used a vanishing theorem of Knop [Kn94].

At last, combining some of the above-mentioned results, we establish the following property suspected by Brion in [Br13]: for saturated $\Gamma$, all the irreducible components of $\mathrm{M}_{\Gamma}$, equipped with their reduced subscheme structure, are affine spaces (see Corollary 4.18). In the above statement, considering the reduced subscheme structure of the irreducible components of $\mathrm{M}_{\Gamma}$ is essential: using the results of the present paper, in [ACF18, § 7.6] we construct examples of saturated monoids $\Gamma$ such that $\mathrm{M}_{\Gamma}$ is a non-reduced point.

This paper is organized as follows. In $\S 1$, we fix notation and conventions used in this paper. In $\S 2$ we gather some basic facts on multiplicity-free affine $G$-varieties and moduli schemes $\mathrm{M}_{\Gamma}$. In $\S 3$ we obtain our description of the $T_{\text {ad }}$-module structure in the tangent space of $\mathrm{M}_{\Gamma}$ at $X_{0}$ whenever $\Gamma$ is saturated. Applications of this description are presented in $\S 4$. Appendix $\mathrm{A}$ lists sign conventions for Chevalley bases of simple Lie algebras used in $\S 3.8$. In appendix $\mathrm{B}$ we present some information on invariants of spherical homogeneous spaces; this material is needed in $\$ \S 4.34 .4$.

Acknowledgements. We thank the referees for their valuable comments and suggestions on a previous version of this paper.

The first author was supported by Dmitry Zimin's "Dynasty" Foundation and the Guest Program of the Max-Planck Institute for Mathematics in Bonn; he also thanks the Institute for Fundamental Science in Moscow for providing excellent working conditions.

The second author was supported by the SFB/TR 12 of the German Research Foundation (DFG). 


\section{Notation And CONVEntions}

Throughout this paper, all topological terms relate to the Zariski topology. All subgroups of algebraic groups are assumed to be closed. The Lie algebras of algebraic groups denoted by capital Latin letters are denoted by the corresponding small Gothic letters. A variety is a separated reduced scheme of finite type. A $K$-variety is a variety equipped with a regular action of an algebraic group $K$. A $K$-isomorphism of two $K$-varieties is a $K$-equivariant isomorphism.

$\mathbb{Z}^{+}=\{z \in \mathbb{Z} \mid z \geq 0\}$

$\mathbb{Q}^{+}=\{q \in \mathbb{Q} \mid q \geq 0\}$

$\mathbb{k}^{\times}$is the multiplicative group of the field $\mathbb{k}$;

$|X|$ is the cardinality of a finite set $X$;

$\langle\cdot, \cdot\rangle$ is the natural pairing between $\operatorname{Hom}_{\mathbb{Z}}(L, \mathbb{Q})$ and $L$, where $L$ is a lattice;

$V^{*}$ is the dual of a vector space $V$;

$K^{0}$ is the connected component of the identity of an algebraic group $K$;

$K_{x}$ is the stabilizer of a point $x$ under an action of a group $K$;

$\mathfrak{X}(K)$ is the character group of a group $K$ (in additive notation);

$k^{\chi}$ is the value of a character $\chi \in \mathfrak{X}(K)$ at an element $k$ of a group $K$;

$Z(K)$ is the center of a group $K$;

$N_{L}(K)$ is the normalizer of a subgroup $K$ in a group $L$;

$\bar{Y}$ is the closure of a subset $Y$ of a scheme $X$;

$\mathbb{k}[X]$ is the algebra of regular functions on a variety $X$;

$\mathbb{k}(X)$ is the field of rational functions on an irreducible variety $X$;

Quot $A$ is the field of fractions of a commutative algebra $A$ with no zero divisors;

Der $A$ is the space of derivations of a commutative algebra $A$;

$[\mathfrak{l}, \mathfrak{l}]$ is the derived subalgebra of a Lie algebra $\mathfrak{l}$;

$\mathcal{O}_{X}$ is the structure sheaf of a scheme $X$;

$T_{x} X$ is the tangent space of a scheme $X$ at a closed point $x \in X$;

$G$ is a connected reductive algebraic group;

$B \subset G$ is a fixed Borel subgroup;

$T \subset B$ is a fixed maximal torus;

$U \subset B$ is the unipotent radical of $B$;

$T_{\mathrm{ad}}=T / Z(G)$ is the adjoint torus;

$(\cdot, \cdot)$ is a fixed inner product on $\mathfrak{X}(T) \otimes_{\mathbb{Z}} \mathbb{Q}$ invariant with respect to the Weyl group $N_{G}(T) / T$

$\Delta \subset \mathfrak{X}(T)$ is the root system of $G$ with respect to $T$;

$\Delta^{+} \subset \Delta$ is the set of positive roots with respect to $B$;

$\Pi \subset \Delta^{+}$is the set of simple roots;

$\alpha^{\vee} \in \operatorname{Hom}_{\mathbb{Z}}(\mathfrak{X}(T), \mathbb{Z})$ is the dual root corresponding to a root $\alpha \in \Delta$;

$\Lambda^{+} \subset \mathfrak{X}(T)$ is the monoid of dominant weights with respect to $B$;

$V(\lambda)$ is the simple $G$-module with highest weight $\lambda \in \Lambda^{+}$;

$U(\mathfrak{g})$ is the universal enveloping algebra of $\mathfrak{g}$.

The lattices $\mathfrak{X}(B)$ and $\mathfrak{X}(T)$ are identified via restricting characters from $B$ to $T$.

The lattice $\mathfrak{X}\left(T_{\text {ad }}\right)$ is canonically identified with $\mathbb{Z} \Pi$.

Highest weight vectors and lowest weight vectors of all simple $G$-modules are considered with respect to $B$. 
For every $\lambda \in \mathfrak{X}(T)$, we set $\lambda^{*}=-w_{0} \lambda$ where $w_{0}$ is the longest element of the Weyl group $N_{G}(T) / T$.

If $V$ is a vector space equipped with an action of a group $K$, then the notation $V^{K}$ stands for the subspace of $K$-invariant vectors and, for every character $\chi$ of $K$, the notation $V_{\chi}^{(K)}$ stands for the subspace of $K$-semi-invariant vectors of weight $\chi$.

Let $K$ be a group and let $K_{1}, K_{2}$ be subgroups of $K$. We write $K=K_{1} \lambda K_{2}$ if $K$ is a semidirect product of $K_{1}, K_{2}$ with $K_{1}$ being a normal subgroup of $K$.

Let $\sigma \in \mathbb{Z} \Pi$ and consider the expression $\sigma=\sum_{\alpha \in \Pi} k_{\alpha} \alpha$, where $k_{\alpha} \in \mathbb{Z}$ for all $\alpha \in \Pi$. The support of $\sigma$ is the set $\operatorname{Supp} \sigma=\left\{\alpha \in \Pi \mid k_{\alpha} \neq 0\right\}$. The type of $\sigma$ is the type of the Dynkin diagram of the set $\operatorname{Supp} \sigma$. When the Dynkin diagram of $\operatorname{Supp} \sigma$ is connected, we number the simple roots in $\operatorname{Supp} \sigma$ as in [Bo68] and denote the $i$ th simple root by $\alpha_{i}$.

For every $\sigma \in \mathbb{Z} \Pi \backslash\{0\}$, the root subsystem of $\Delta$ with set of simple roots Supp $\sigma$ is denoted by $\Delta_{\sigma}$.

For every subset $F \subset \mathfrak{X}(T)$, we set $F^{\perp}=\left\{\alpha \in \Pi \mid\left\langle\alpha^{\vee}, \lambda\right\rangle=0\right.$ for all $\left.\lambda \in F\right\}$. By abuse of notation, for a single element $\lambda \in \mathfrak{X}(T)$ we write $\lambda^{\perp}$ instead of $\{\lambda\}^{\perp}$.

For every $\alpha \in \Delta$, the image of $\alpha^{\vee}$ in $\mathfrak{t}$ is denoted by $h_{\alpha}$.

For every $\alpha \in \Delta$, we fix a nonzero root vector $e_{\alpha} \in \mathfrak{g}$ of weight $\alpha$ with respect to the adjoint action of $T$. Moreover, we assume that the set $\left\{h_{\alpha} \mid \alpha \in \Pi\right\} \cup\left\{e_{\alpha} \mid \alpha \in \Delta\right\}$ is a Chevalley basis of the semisimple Lie algebra $[\mathfrak{g}, \mathfrak{g}]$ (for details on Chevalley bases see [Ca89, $\S \S 4.1-4.2])$.

For every $\alpha, \beta \in \Delta$ with $\alpha+\beta \in \Delta$ we let $N_{\alpha, \beta} \in\{ \pm 1, \pm 2, \pm 3, \pm 4\}$ be the corresponding structure constant so that $\left[e_{\alpha}, e_{\beta}\right]=N_{\alpha, \beta} e_{\alpha+\beta}$. One has $\left|N_{\alpha, \beta}\right|=p+1$ where $p$ is the largest integer such that $\beta-p \alpha \in \Delta$.

Let $Q$ be a finite-dimensional vector space over $\mathbb{Q}$.

A subset $\mathcal{C} \subset Q$ is called a (finitely generated convex) cone if there are finitely many elements $q_{1}, \ldots, q_{s} \in Q$ such that $\mathcal{C}=\mathbb{Q}^{+} q_{1}+\ldots+\mathbb{Q}^{+} q_{s}$.

The dimension of a cone is the dimension of its linear span.

The dual cone of a cone $\mathcal{C} \subset Q$ is the cone

$$
\mathcal{C}^{\vee}=\left\{\xi \in Q^{*} \mid \xi(q) \geq 0 \text { for all } q \in \mathcal{C}\right\} .
$$

One always has $\left(\mathcal{C}^{\vee}\right)^{\vee}=\mathcal{C}$.

A face of a cone $\mathcal{C} \subset Q$ is a subset $\mathcal{F} \subset \mathcal{C}$ of the form

$$
\mathcal{F}=\mathcal{C} \cap\{q \in Q \mid \xi(q)=0\}
$$

for some $\xi \in \mathcal{C}^{\vee}$. Each face of $\mathcal{C}$ is again a cone.

An extremal ray of a cone $\mathcal{C}$ is a face of dimension 1 having the form $\mathbb{Q}^{+} q$ for some $q \in Q \backslash\{0\}$.

\section{BASIC MATERIAL}

In this section, we collect basic material on multiplicity-free affine $G$-varieties and on Alexeev and Brion's moduli schemes.

\subsection{Spherical $G$-varieties and multiplicity-free affine $G$-varieties.}

Definition 2.1. A $G$-variety $X$ is said to be spherical if $X$ is normal and possesses a dense (and hence open) $B$-orbit. 
It follows from the definition that every spherical $G$-variety is irreducible.

Given a $G$-variety $X$, the algebra $\mathbb{k}[X]$ is naturally equipped with the $G$-module structure given by $(g f)(x)=f\left(g^{-1} x\right)$ for all $g \in G, f \in \mathbb{k}[X]$, and $x \in X$.

Definition 2.2. An affine $G$-variety $X$ is said to be multiplicity-free if $X$ is irreducible and every simple $G$-module occurs in $\mathbb{k}[X]$ with multiplicity at most 1.

Theorem 2.3 ([ViK78, Theorem 2]). Let $X$ be an irreducible affine G-variety. The following conditions are equivalent:

(1) $X$ is multiplicity-free.

(2) $X$ possesses a dense B-orbit.

Corollary 2.4. Let $X$ be an affine G-variety. The following conditions are equivalent:

(1) $X$ is spherical.

(2) $X$ is multiplicity-free and normal.

2.2. The weight monoid. Let $X$ be a multiplicity-free affine $G$-variety.

Definition 2.5. The weight monoid of $X$, denoted by $\Gamma_{X}$, is the set of all $\lambda \in \Lambda^{+}$such that $\mathbb{k}[X]$ contains a simple $G$-submodule isomorphic to $V(\lambda)$.

Remark 2.6. As $\mathbb{k}[X]$ is an integral domain, the product of two highest weight vectors in $\mathbb{k}[X]$ is nonzero and hence again a highest weight vector. It follows that $\Gamma_{X}$ is indeed a submonoid in $\Lambda^{+}$.

For every $\lambda \in \Gamma_{X}$, we let $\mathbb{k}[X]_{\lambda}$ denote the simple $G$-submodule of $\mathbb{k}[X]$ isomorphic to $V(\lambda)$, so that

$$
\mathbb{k}[X]=\bigoplus_{\lambda \in \Gamma_{X}} \mathbb{k}[X]_{\lambda}
$$

Given a submonoid $\Gamma \subset \mathfrak{X}(T)$, let $\mathbb{k}[\Gamma]$ denote the "semigroup algebra" of $\Gamma$, that is, the algebra with basis $\left\{u_{\lambda} \mid \lambda \in \Gamma\right\}$ and multiplication given by $u_{\lambda} u_{\mu}=u_{\lambda+\mu}$ for all $\lambda, \mu \in \Gamma$. We equip $\mathbb{k}[\Gamma]$ with an action of $T$ given by the formula $t \cdot u_{\lambda}=t^{\lambda} u_{\lambda}$ for all $t \in T$ and $\lambda \in \Gamma$. Clearly, the multiplication of $\mathbb{k}[\Gamma]$ is $T$-equivariant.

Proposition 2.7 ([요 , Theorem 2]). There is a T-equivariant isomorphism $\mathbb{k}[X]^{U} \simeq$ $\mathbb{k}\left[\Gamma_{X}\right]$.

Corollary 2.8. The monoid $\Gamma_{X}$ is finitely generated.

Proof. As the algebra $\mathbb{k}[X]$ is finitely generated, so is $\mathbb{k}[X]^{U}$ by [Ha67, Theorem 3.1] (see also [Po86, Corollary 4 of Theorem 4]). It remains to apply Proposition 2.7.

Proposition 2.9. The algebra $\mathbb{k}[X]$ is integrally closed if and only if so is $\mathbb{k}[X]^{U}$.

Proof. This is a particular case of Vust's normality criterion [Vu76, §1.2, Theorem 1] (see also [Po86, Corollary of Theorem 6]).

Definition 2.10. A monoid $\Gamma \subset \mathfrak{X}(T)$ is said to be saturated if it satisfies the equality $\Gamma=\mathbb{Z} \Gamma \cap \mathbb{Q}^{+} \Gamma$ in $\mathfrak{X}(T) \otimes_{\mathbb{Z}} \mathbb{Q}$.

Proposition 2.11. The following conditions are equivalent:

(1) $X$ is normal (and hence spherical). 
(2) $\Gamma_{X}$ is saturated.

Proof. By KKMS73, Ch. I, $\S 1$, Lemma 1], the algebra $\mathbb{k}\left[\Gamma_{X}\right]$ is integrally closed if and only if $\Gamma_{X}$ is saturated. Now the claim follows from Propositions 2.7 and 2.9 .

2.3. The root monoid and related invariants. Let $X$ be a multiplicity-free affine $G$-variety.

Definition 2.12. The root monoid of $X$, denoted by $\Xi_{X}$, is the monoid in $\mathfrak{X}(T)$ generated by all expressions $\lambda+\mu-\nu$ with $\lambda, \mu, \nu \in \Gamma_{X}$ such that the linear span of $\mathbb{k}[X]_{\lambda} \cdot \mathbb{k}[X]_{\mu}$ contains $\mathbb{k}[X]_{\nu}$.

It follows from the definition that $\Xi_{X}$ is a submonoid of $\mathbb{Z}^{+} \Pi$. It is known that $\Xi_{X}$ is finitely generated, see AB05, Proposition 2.13].

Let $\Xi_{X}^{\text {sat }}$ denote the saturation of $\Xi_{X}$, that is, $\Xi_{X}^{\text {sat }}=\mathbb{Z} \Xi_{X} \cap \mathbb{Q}^{+} \Xi_{X}$.

Theorem 2.13 (see [Kn96, Theorem 1.3]). The monoid $\Xi_{X}^{\text {sat }}$ is free.

According to Theorem 2.13, let $\bar{\Sigma}_{X} \subset \mathbb{Z}^{+} \Pi$ be the set of free generators of the monoid $\Xi_{X}^{\text {sat }}$, that is, the linearly independent set such that

$$
\Xi_{X}^{\text {sat }}=\mathbb{Z}^{+} \bar{\Sigma}_{X}
$$

Along with the set $\bar{\Sigma}_{X}$, we shall also consider the set $\Sigma_{X}$ consisting of primitive elements $\sigma$ of the lattice $\mathbb{Z} \Gamma_{X}$ such that $\mathbb{Q}^{+} \sigma$ is an extremal ray of the cone $\mathbb{Q}^{+} \Xi_{X} \subset \mathbb{Z} \Gamma_{X} \otimes_{\mathbb{Z}} \mathbb{Q}$. Elements of $\Sigma_{X}$ are called spherical roots of $X$.

2.4. The $G$-variety $X_{0}$. From now on until the end of $\S 2.8, \Gamma \subset \Lambda^{+}$is an arbitrary finitely generated monoid.

Fix an arbitrary finite generating system $\mathrm{E} \subset \Gamma$ and consider the $G$-module

$$
V=V(\mathrm{E})=\bigoplus_{\lambda \in \mathrm{E}} V(\lambda)^{*}
$$

For every $\lambda \in \mathrm{E}$, fix a lowest weight vector $v_{\lambda} \in V(\lambda)^{*}$. Put

$$
x_{0}=\sum_{\lambda \in \mathrm{E}} v_{\lambda}, \quad O=G x_{0}, \quad \text { and } \quad X_{0}=\bar{O} \subset V .
$$

Theorem 2.14 ([ViP72, Theorem 6]). The following assertions hold:

(a) up to a $G$-isomorphism, the $G$-variety $X_{0}$ is independent of the choice of $\mathrm{E}$;

(b) $X_{0}$ is a multiplicity-free affine $G$-variety;

(c) $\Gamma_{X_{0}}=\Gamma$;

(d) $\Xi_{X_{0}}=\{0\}$, that is, the linear span of $\mathbb{k}\left[X_{0}\right]_{\lambda} \cdot \mathbb{k}\left[X_{0}\right]_{\mu}$ coincides with $\mathbb{k}\left[X_{0}\right]_{\lambda+\mu}$ for all $\lambda, \mu \in \Gamma_{X}$.

2.5. The definition of $\mathrm{M}_{\Gamma}$. Consider the $G$-module

$$
A_{\Gamma}=\bigoplus_{\lambda \in \Gamma} V(\lambda)
$$

For every $\lambda \in \Gamma$, fix a highest weight vector $u_{\lambda} \in V(\lambda)$. Then $A_{\Gamma}^{U}=\bigoplus_{\lambda \in \Gamma} \mathbb{k} u_{\lambda}$. We equip $A_{\Gamma}^{U}$ with an algebra structure by setting

$$
u_{\lambda} \cdot u_{\mu}=u_{\lambda+\mu} \text { for all } \lambda, \mu \in \Gamma \text {. }
$$


Thus we get a canonical identification

$$
A_{\Gamma}^{U} \simeq \mathbb{k}[\Gamma]
$$

Every scheme $S$ is naturally equipped with the sheaf of $\mathcal{O}_{S^{-}} G$-modules $\mathcal{O}_{S} \otimes_{\mathbb{k}} A_{\Gamma}$.

We consider the contravariant functor

$$
\mathcal{M}_{\Gamma}:(\text { Schemes }) \rightarrow(\text { Sets })
$$

assigning to each scheme $S$ the set of $\mathcal{O}_{S^{-}} G$-algebra structures on the sheaf $\mathcal{O}_{S} \otimes_{\mathbb{k}} A_{\Gamma}$ that extend the multiplication (2.2) on $A_{\Gamma}^{U}$. By [AB05, Proposition 2.10], this definition of $\mathcal{M}_{\Gamma}$ agrees with that given in [AB05, Definition 1.11], see also [Br13, $\S 4.3$.

The following result is a consequence of [AB05, Theorems 1.12 and 2.7], see also [Br13, $\S 4.3]$.

Theorem 2.15. The functor $\mathcal{M}_{\Gamma}$ is represented by an affine scheme $\mathrm{M}_{\Gamma}$ of finite type.

Let $\operatorname{ML}\left(A_{\Gamma}\right)$ denote the set of all $G$-equivariant multiplication laws on $A_{\Gamma}$ extending the multiplication $(2.2)$ on $A_{\Gamma}^{U}$.

Corollary 2.16. The set of closed points of $\mathrm{M}_{\Gamma}$ is in bijection with the set $\operatorname{ML}\left(A_{\Gamma}\right)$.

2.6. Relation of $\mathrm{M}_{\Gamma}$ to multiplicity-free affine $G$-varieties with weight monoid $\Gamma$. Consider a multiplicity-free affine $G$-variety $X$ with weight monoid $\Gamma$. In view of Proposition [2.7, there is a $T$-equivariant algebra isomorphism

$$
\tau: \mathbb{k}[X]^{U} \stackrel{\sim}{\rightarrow} \mathbb{k}[\Gamma] .
$$

Identifying $\mathbb{k}[\Gamma]$ with $A_{\Gamma}^{U}$ via (2.3) , we get a $T$-equivariant isomorphism $\mathbb{k}[X]^{U} \stackrel{\sim}{\rightarrow} A_{\Gamma}^{U}$. Clearly, the latter isomorphism uniquely extends to a $G$-module isomorphism

$$
\mathbb{k}[X] \stackrel{\sim}{\rightarrow} A_{\Gamma} .
$$

Transferring the algebra structure from $\mathbb{k}[X]$ to $A_{\Gamma}$ via isomorphism (2.5), we obtain a $G$-equivariant multiplication law on $A_{\Gamma}$ extending the multiplication of $A_{\Gamma}^{U}$.

Let $X_{1}, X_{2}$ be two multiplicity-free affine $G$-varieties with weight monoid $\Gamma$ and fix $T$-equivariant isomorphisms $\tau_{i}: \mathbb{k}\left[X_{i}\right]^{U \stackrel{\sim}{\rightarrow}} \mathbb{k}[\Gamma](i=1,2)$. We say that the pairs $\left(X_{1}, \tau_{1}\right)$ and $\left(X_{2}, \tau_{2}\right)$ are equivalent if there is a $G$-equivariant isomorphism $\mathbb{k}\left[X_{1}\right] \stackrel{\sim}{\rightarrow} \mathbb{k}\left[X_{2}\right]$ such that the induced $T$-equivariant isomorphism $\mathbb{k}\left[X_{1}\right]^{U} \stackrel{\sim}{\rightarrow} \mathbb{k}\left[X_{2}\right]^{U}$ fits into a commutative diagram

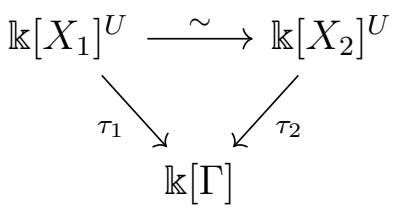

Combining the above material with Corollary 2.16, we get

Proposition 2.17. The closed points of $\mathrm{M}_{\Gamma}$ are in bijection with the equivalence classes of pairs $(X, \tau)$, where $X$ is a multiplicity-free affine $G$-variety with weight monoid $\Gamma$ and $\tau: \mathbb{k}[X]^{U} \stackrel{\sim}{\rightarrow} \mathbb{k}[\Gamma]$ is a $T$-equivariant algebra isomorphism. 
2.7. Basic facts on the action of $T_{\mathrm{ad}}$ on $\mathrm{M}_{\Gamma}$. Let $A_{\Gamma}$ be as in $\S 2.5$. In view of (2.1), every multiplication law $m \in \operatorname{ML}\left(A_{\Gamma}\right)$ can be expressed as the sum

$$
m=\sum_{\lambda, \mu, \nu \in \Gamma} m_{\lambda, \mu}^{\nu}
$$

where each component $m_{\lambda, \mu}^{\nu}: V(\lambda) \otimes V(\mu) \rightarrow V(\nu)$ is a $G$-module homomorphism.

Proposition 2.18 ([AB05, Proposition 2.11]). Modulo the identification of Corollary 2.16, the action of $T_{\mathrm{ad}}$ on the set of closed points of $\mathrm{M}_{\Gamma}$ is described as follows:

$$
(t \cdot m)_{\lambda, \mu}^{\nu}=t^{\nu-\lambda-\mu} m_{\lambda, \mu}^{\nu} \text { for all } t \in T_{\mathrm{ad}}, m \in \operatorname{ML}\left(A_{\Gamma}\right) \text {. }
$$

Corollary 2.19. Modulo the identification of Proposition 2.17, suppose that (the equivalence classes of $)$ two closed points $\left(X_{1}, \tau_{1}\right)$ and $\left(X_{2}, \tau_{2}\right)$ of $\mathrm{M}_{\Gamma}$ lie in the same $T_{\mathrm{ad}}$-orbit. Then $X_{1}$ and $X_{2}$ are $G$-isomorphic.

Theorem 2.20 ([AB05, Theorem 1.12 and Lemma 2.2]). The G-isomorphism classes of multiplicity-free affine $G$-varieties with weight monoid $\Gamma$ are in bijection with the $T_{\text {ad }}$-orbits in $\mathrm{M}_{\Gamma}$.

The following result is a consequence of Corollary 2.19 and Theorem 2.20,

Corollary 2.21. Suppose that $X$ is a multiplicity-free affine G-variety with weight monoid $\Gamma$. Then, modulo the identification of Proposition 2.17, the closed points of the $T_{\text {ad }}$-orbit in $\mathrm{M}_{\Gamma}$ corresponding to $X$ are all (equivalence classes of) pairs of the form $(X, \tau)$.

According to Theorem 2.20, for every multiplicity-free affine $G$-variety $X$ with weight monoid $\Gamma$ we let $C_{X}$ denote the closure of the $T_{\text {ad }}$-orbit in $\mathrm{M}_{\Gamma}$ corresponding to the $G$ isomorphism class of $X$.

Since $\Xi_{X_{0}}=\{0\}$ by Theorem 2.14(d), it follows from (2.6) and Corollary 2.21 that the $T_{\text {ad }}$-orbit in $\mathrm{M}_{\Gamma}$ corresponding to $X_{0}$ is a $T_{\text {ad }}$-fixed (closed) point. In what follows, by abuse of notation, we denote this point by $X_{0}$. In particular, $C_{X_{0}}=\left\{X_{0}\right\}$.

Theorem 2.22 ([AB05, Theorem 2.7]). The $T_{\text {ad }}$-fixed point $X_{0} \in \mathrm{M}_{\Gamma}$ is the unique closed $T_{\text {ad }}$-orbit in $\mathrm{M}_{\Gamma}$. Equivalently, $X_{0}$ is contained in each $T_{\text {ad }}$-orbit closure in $\mathrm{M}_{\Gamma}$.

Theorem 2.23 ([AB05, Proposition 2.13]). Let $X$ be a multiplicity-free affine G-variety with weight monoid $\Gamma$. The $T_{\mathrm{ad}}$-orbit closure $C_{X} \subset \mathrm{M}_{\Gamma}$, equipped with its reduced subscheme structure, is a multiplicity-free affine $T_{\text {ad }}$-variety whose weight monoid is $\Xi_{X}$.

Corollary 2.24. Under the hypotheses of Theorem 2.23, the tangent space $T_{X_{0}} C_{X}$ is a multiplicity-free $T_{\mathrm{ad}}$-module whose set of weights is

$$
\left\{-\tau \mid \tau \text { is an indecomposable element of } \Xi_{X}\right\} .
$$

Proof. By Theorem 2.23, $C_{X}$ is a multiplicity-free affine $T_{\text {ad-variety with weight }}$ monoid $\Xi_{X}$, so that $\mathbb{k}\left[C_{X}\right]=\bigoplus_{\xi \in \Xi_{X}} \mathbb{k}\left[C_{X}\right]_{\xi}^{\left(T_{\text {ad }}\right)}$. Recall from Theorem 2.22 that $X_{0}$ is the unique $T_{\text {ad }}$-fixed closed point in $\mathrm{M}_{\Gamma_{X}}$ (and hence in $C_{X}$ ), therefore it corresponds to the maximal ideal

$$
I=\bigoplus_{\xi \in \Xi_{X} \backslash\{0\}} \mathbb{k}\left[C_{X}\right]_{\xi}^{\left(T_{\text {ad }}\right)} \subset \mathbb{k}\left[C_{X}\right]
$$

Now the assertion follows from the $T_{\text {ad }}$-equivariant isomorphism $T_{X_{0}} C_{X} \simeq\left(I / I^{2}\right)^{*}$. 
2.8. Characterizations of $T_{X_{0}} \mathrm{M}_{\Gamma}$. In this subsection we present general facts on the $T_{\text {ad }}$-module structure of the tangent space $T_{X_{0}} \mathrm{M}_{\Gamma}$.

Let $\mathrm{E}, V, v_{\lambda}(\lambda \in \mathrm{E}), x_{0}, O$, and $X_{0}$ be as in $\S 2.4$. Let $a_{G}^{*}:(g, v) \mapsto g * v$ be the natural action of $G$ on $V$. Given $t \in T$, let $\bar{t}$ denote the image of $t$ in $T_{\text {ad }}$.

According to $\left[\mathrm{AB} 05, \S 2.1\right.$, we define an action $a_{\mathrm{ad}}^{*}:(\bar{t}, v) \mapsto \bar{t} * v$ of $T_{\mathrm{ad}}$ on $V$ in the following way. For every $\lambda \in \mathrm{E}$ and $v \in V(\lambda)^{*}$, we set

$$
\bar{t} * v=t^{-\lambda}\left(t^{-1} * v\right),
$$

and then extend the action to the whole $V$. Note that

$$
V^{T_{\mathrm{ad}}}=\bigoplus_{\lambda \in \mathrm{E}} \mathbb{k} v_{\lambda}
$$

We introduce the semi-direct product $\widetilde{G}=G \lambda T_{\text {ad }}$ given by $\bar{t} g \bar{t}^{-1}=t^{-1} g t$ for all $t \in T$ and $g \in G$. Then the actions $a_{G}^{*}$ and $a_{\text {ad }}^{*}$ extend to an action of $\widetilde{G}$ on $V$, which will be denoted by $a^{*}$. Observe that $x_{0} \in V^{T_{\text {ad }}}$ by (2.7), and so the orbit $G x_{0}$ is $T_{\text {ad }}$-stable and hence $\widetilde{G}$-stable. It follows that $X_{0}$ is $\widetilde{G}$-stable.

All actions of $\widetilde{G}$ (resp. $T_{\mathrm{ad}}$ ) that will be considered in the remaining part of this subsection are induced by the action $a^{*}$ (resp. $a_{\mathrm{ad}}^{*}$ ) on $V$ and its restriction to $X_{0}$.

Let $\Omega_{V}^{1}$ (resp. $\Omega_{X_{0}}^{1}$ ) denote the sheaf of differential 1-forms on $V$ (resp. $X_{0}$ ). Consider the canonical closed immersion $i: X_{0} \hookrightarrow V$ and let $\mathcal{I}$ be the corresponding ideal sheaf. By [Ha77, Proposition II.8.12] or [Li02, Proposition 6.1.24(d)], there is an exact sequence of coherent sheaves of $\mathcal{O}_{X_{0}}$-modules

$$
i^{*}\left(\mathcal{I} / \mathcal{I}^{2}\right) \rightarrow i^{*} \Omega_{V}^{1} \rightarrow \Omega_{X_{0}}^{1} \rightarrow 0 .
$$

We note that $i^{*} \Omega_{V}^{1} \simeq \mathcal{O}_{X_{0}} \otimes_{\mathbb{k}} V^{*}$ as $\mathcal{O}_{X_{0}}$-modules. Applying $H_{o m}{\mathcal{O}_{X_{0}}}\left(-, \mathcal{O}_{X_{0}}\right)$ to the above exact sequence, we obtain an exact sequence of coherent sheaves of $\mathcal{O}_{X_{0}}$-modules

$$
0 \rightarrow \mathcal{T}_{X_{0}} \rightarrow \mathcal{O}_{X_{0}} \otimes_{\mathbb{k}} V \rightarrow \mathcal{N}_{X_{0}}
$$

where

$$
\mathcal{T}_{X_{0}}=\operatorname{Hom}_{\mathcal{O}_{X_{0}}}\left(\Omega_{X_{0}}^{1}, \mathcal{O}_{X_{0}}\right)
$$

is the tangent sheaf of $X_{0}$ (that is, the sheaf of $\mathbb{k}$-derivations of the sheaf $\mathcal{O}_{X_{0}}$ ) and

$$
\mathcal{N}_{X_{0}}=\operatorname{Hom}_{\mathcal{O}_{X_{0}}}\left(i^{*}\left(\mathcal{I} / \mathcal{I}^{2}\right), \mathcal{O}_{X_{0}}\right)
$$

is the normal sheaf of $X_{0}$ in $V$. Taking global sections in (2.8) yields an exact sequence of $\mathbb{k}\left[X_{0}\right]-\widetilde{G}$-modules

$$
0 \rightarrow H^{0}\left(X_{0}, \mathcal{T}_{X_{0}}\right) \rightarrow H^{0}\left(X_{0}, \mathcal{O}_{X_{0}} \otimes_{\mathbb{k}} V\right) \rightarrow H^{0}\left(X_{0}, \mathcal{N}_{X_{0}}\right) \rightarrow T^{1}\left(X_{0}\right) \rightarrow 0,
$$

where $T^{1}\left(X_{0}\right)$, called the space of infinitesimal deformations of $X_{0}$, is by definition the cokernel of the map $H^{0}\left(X_{0}, \mathcal{O}_{X_{0}} \otimes_{\mathbb{k}} V\right) \rightarrow H^{0}\left(X_{0}, \mathcal{N}_{X_{0}}\right)$ in (2.9) (see [Ha77, Exercise III.9.8]).

The following characterization of the tangent space $T_{X_{0}} \mathrm{M}_{\Gamma}$, which is implicitly contained in AB05, has already been mentioned in [Br13, Subsection 4.3]. For the reader's convenience, we provide it together with a proof.

Proposition 2.25. There is a $T_{\mathrm{ad}}$-module isomorphism $T_{X_{0}} \mathrm{M}_{\Gamma} \simeq T^{1}\left(X_{0}\right)^{G}$. 
Proof. Applying [AB05, Proposition 2.8], we obtain an exact sequence of $T_{\mathrm{ad}}$-modules

$$
0 \rightarrow \operatorname{Der}^{G}\left(\mathbb{k}\left[X_{0}\right]\right) \rightarrow \operatorname{Der}^{T}\left(\mathbb{k}\left[X_{0}\right]^{U}\right) \rightarrow T_{X_{0}} \mathrm{M}_{\Gamma} \rightarrow T^{1}\left(X_{0}\right)^{G} \rightarrow T^{1}\left(X_{0} / / U\right)^{T} \rightarrow 0,
$$

where $X_{0} / / U=\operatorname{Spec} \mathbb{k}\left[X_{0}\right]^{U}$. By $\left[\mathrm{AB05}\right.$, Proposition 1.15(ii)], $T^{1}\left(X_{0} / / U\right)^{T}$ is trivial. Therefore it remains to prove that the map $\operatorname{Der}^{G}\left(\mathbb{k}\left[X_{0}\right]\right) \rightarrow \operatorname{Der}^{T}\left(\mathbb{k}\left[X_{0}\right]^{U}\right)$, given by restricting derivations from $\mathbb{k}\left[X_{0}\right]$ to $\mathbb{k}\left[X_{0}\right]^{U}$, is surjective (and hence an isomorphism). To this end, let $B$ act on $\mathbb{k}[G]$ by right multiplication and on $\mathbb{k}\left[X_{0}\right]^{U}$ in such a way that each $T$-eigenvector of weight $\lambda$ is multiplied by the character $-\lambda^{*}$. Then there is a $G$ equivariant isomorphism of algebras

$$
\mathbb{k}\left[X_{0}\right] \simeq\left(\mathbb{k}[G] \otimes_{\mathbb{k}} \mathbb{k}\left[X_{0}\right]^{U}\right)^{B},
$$

where $B$-invariants are taken with respect to the diagonal action of $B$ and the action of $G$ on the right-hand side is induced by that on $\mathbb{k}[G]$ by left multiplication. It is clear from (2.10) that every $T$-equivariant derivation of $\mathbb{k}\left[X_{0}\right]^{U}$ extends to a $G$-equivariant derivation of $\mathbb{k}\left[X_{0}\right]$.

Corollary 2.26. There is an exact sequence of $T_{\mathrm{ad}}$-modules

$$
0 \rightarrow H^{0}\left(X_{0}, \mathcal{T}_{X_{0}}\right)^{G} \rightarrow H^{0}\left(X_{0}, \mathcal{O}_{X_{0}} \otimes_{\mathbb{k}} V\right)^{G} \rightarrow H^{0}\left(X_{0}, \mathcal{N}_{X_{0}}\right)^{G} \rightarrow T_{X_{0}} \mathrm{M}_{\Gamma} \rightarrow 0
$$

Proof. This follows by taking $G$-invariants in (2.9) and applying Proposition 2.25.

Given a smooth open subset $Y \subset X_{0}$, the restrictions to $Y$ of all the sheaves appearing in (2.8) are well known to be locally free, hence they may be regarded as the sheaves of sections of vector bundles on $Y$. More precisely, $\left.\mathcal{T}_{X_{0}}\right|_{Y}\left(\operatorname{resp} .\left.\quad\left(\mathcal{O}_{X_{0}} \otimes_{\mathbb{k}} V\right)\right|_{Y},\left.\mathcal{N}_{X_{0}}\right|_{Y}\right)$ will be regarded as the sheaf of sections of the tangent bundle of $Y$ (resp. trivial bundle $Y \times V$, normal bundle of $Y$ in $V$ ). If, in addition, $Y$ is $G$-stable, then the three vector bundles are $G$-linearized in a natural way.

Proposition 2.27. The exact sequence of $T_{\mathrm{ad}}$-modules

$$
0 \rightarrow H^{0}\left(O,\left.\mathcal{T}_{X_{0}}\right|_{O}\right)^{G} \rightarrow H^{0}\left(O,\left.\left(\mathcal{O}_{X_{0}} \otimes_{\mathbb{k}} V\right)\right|_{O}\right)^{G} \rightarrow H^{0}\left(O,\left.\mathcal{N}_{X_{0}}\right|_{O}\right)^{G}
$$

identifies with

$$
0 \rightarrow\left(\mathfrak{g} x_{0}\right)^{G_{x_{0}}} \rightarrow V^{G_{x_{0}}} \rightarrow\left(V / \mathfrak{g} x_{0}\right)^{G_{x_{0}}}
$$

Proof. As $O$ is $G$-homogeneous, for every $G$-linearized vector bundle $p: F \rightarrow O$ the space of its $G$-invariant sections is canonically isomorphic to $\left(p^{-1}\left(x_{0}\right)\right)^{G_{x_{0}}}$. Applying this to our three vector bundles yields the claim.

By [Br13, Lemma 3.9], for every coherent sheaf $\mathcal{F}$ on $X_{0}$ the restriction map $H^{0}\left(X_{0}, \mathcal{G}\right) \rightarrow H^{0}\left(O,\left.\mathcal{G}\right|_{O}\right)$ is injective, where $\mathcal{G}=H_{o m_{\mathcal{O}_{X_{0}}}}\left(\mathcal{F}, \mathcal{O}_{X_{0}}\right)$. Combining this with Corollary 2.26 and Proposition 2.27 we obtain the following result.

Proposition 2.28. There is a commutative diagram of $T_{\mathrm{ad}}$-modules

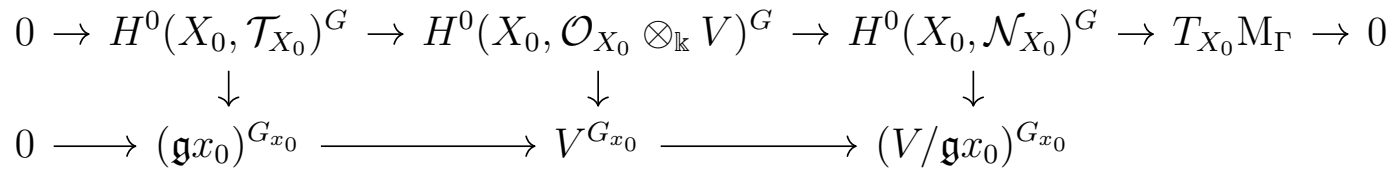

where the rows are exact and the vertical arrows are injective maps. 
Proposition 2.29. There is an exact sequence of $T_{\mathrm{ad}}$-modules

$$
0 \rightarrow H^{0}\left(X_{0}, \mathcal{N}_{X_{0}}\right)^{\widetilde{G}} \rightarrow H^{0}\left(X_{0}, \mathcal{N}_{X_{0}}\right)^{G} \rightarrow T_{X_{0}} \mathrm{M}_{\Gamma} \rightarrow 0 .
$$

Remark 2.30. $H^{0}\left(X_{0}, \mathcal{N}_{X_{0}}\right)^{\widetilde{G}}=\left[H^{0}\left(X_{0}, \mathcal{N}_{X_{0}}\right)^{G}\right]^{T_{\text {ad }}}$.

Proof of Proposition 2.29. The claim will follow as soon as we show that the image of the map

$$
H^{0}\left(X_{0}, \mathcal{O}_{X_{0}} \otimes_{\mathbb{k}} V\right)^{G} \rightarrow H^{0}\left(X_{0}, \mathcal{N}_{X_{0}}\right)^{G}
$$

in (2.11) coincides with $H^{0}\left(X_{0}, \mathcal{N}_{X_{0}}\right)^{\widetilde{G}}$. Since $G_{x_{0}}$ contains a maximal unipotent subgroup of $G$, it follows that the space $V^{G_{x_{0}}}$ is just the linear span of all vectors $v_{\lambda}$ with $\lambda \in \mathrm{E}$, which implies

$$
V^{G_{x_{0}}}=V^{T_{\text {ad }}}
$$

by (2.7). Therefore $T_{\text {ad }}$ acts trivially on $V^{G_{x_{0}}}$ and hence on $H^{0}\left(X_{0}, \mathcal{O}_{X_{0}} \otimes_{\mathbb{k}} V\right)^{G}$. Thus the image of the map (2.12) is contained in $H^{0}\left(X_{0}, \mathcal{N}_{X_{0}}\right)^{\widetilde{G}}$, and so there is a commutative diagram of vector spaces

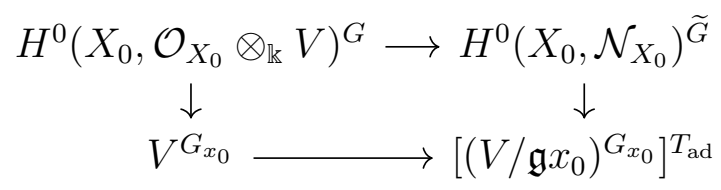

where the vertical arrows are injective maps. Further, note that

$$
H^{0}\left(X_{0}, \mathcal{O}_{X_{0}} \otimes_{\mathbb{k}} V\right) \simeq \mathbb{k}\left[X_{0}\right] \otimes_{\mathbb{k}} V
$$

and $\mathbb{k}\left[X_{0}\right] \otimes_{\mathbb{k}} V$ contains $V(\lambda) \otimes V(\lambda)^{*}$ as a $G$-submodule for every $\lambda \in E$ E. Since $\operatorname{dim}\left(V(\lambda) \otimes V(\lambda)^{*}\right)^{G} \geq 1$ for every $\lambda \in \Lambda^{+}$, it follows that $\operatorname{dim} H^{0}\left(X_{0}, \mathcal{O}_{X_{0}} \otimes_{\mathbb{k}} V\right)^{G} \geq|\mathrm{E}|$. On the other hand, it is clear that $\operatorname{dim} V^{G_{x_{0}}}=|\mathrm{E}|$. Consequently, the left vertical arrow in (2.14) is an isomorphism. At last, the surjectivity of the natural map $V^{T_{\text {ad }}} \rightarrow\left(V / \mathfrak{g} x_{0}\right)^{T_{\text {ad }}}$ along with (2.13) implies that the lower horizontal arrow in (2.14) is a surjective map. The latter already suffices to conclude that the map given by the upper horizontal arrow in (2.14) is also surjective.

Combining Proposition 2.29 with Remark 2.30 we obtain

Corollary 2.31. All $T_{\mathrm{ad}}$-weights of $T_{X_{0}} \mathrm{M}_{\Gamma}$ are nonzero.

\section{The tangent space of $\mathrm{M}_{\Gamma}$ AT $X_{0}$}

Throughout this section, we fix the following notation:

$\Gamma \subset \Lambda^{+}$is an arbitrary finitely generated and saturated monoid;

$\mathcal{L}=\operatorname{Hom}_{\mathbb{Z}}(\mathbb{Z} \Gamma, \mathbb{Z})$

$\mathcal{Q}=\mathcal{L} \otimes_{\mathbb{Z}} \mathbb{Q}=\operatorname{Hom}_{\mathbb{Z}}(\mathbb{Z} \Gamma, \mathbb{Q})$

$\mathcal{K} \subset \mathcal{Q}$ is the cone dual to $\mathbb{Q}^{+} \Gamma$;

$\mathcal{K}^{1} \subset \mathcal{K}$ is the set of primitive elements $q$ in $\mathcal{L}$ such that $\mathbb{Q}^{+} q$ is an extremal ray of $\mathcal{K}$;

$\iota: \operatorname{Hom}_{\mathbb{Z}}(\mathfrak{X}(T), \mathbb{Q}) \rightarrow \mathcal{Q}$ is the natural restriction map.

For every $\sigma \in \mathbb{Z} \Gamma$ we define the set

$$
\mathcal{K}^{1}(\sigma)=\left\{\varrho \in \mathcal{K}^{1} \mid\langle\varrho, \sigma\rangle>0\right\} .
$$


3.1. Statement of the main result. We first describe the set $\bar{\Sigma}(G)$. By definition, an element $\sigma \in \mathfrak{X}(T)$ belongs to $\bar{\Sigma}(G)$ if and only if $\sigma \in \mathbb{Z}^{+} \Pi \backslash\{0\}$ and the expression of $\sigma$ as a linear combination of the simple roots in $\operatorname{Supp} \sigma$ appears in Table 1. (In row 3 of this table, $\alpha$ and $\beta$ are the two distinct simple roots in $\operatorname{Supp} \sigma$.)

TABle 1. The Set $\bar{\Sigma}(G)$

\begin{tabular}{|c|c|c|c|c|}
\hline No. & $\begin{array}{l}\text { Type of } \\
\text { Supp } \sigma\end{array}$ & $\sigma$ & $\Pi_{\sigma}$ & Note \\
\hline 1 & $\overline{A_{1}}$ & $\alpha_{1}$ & $\varnothing$ & \\
\hline 2 & $\overline{A_{1}}$ & $2 \alpha_{1}$ & $\varnothing$ & \\
\hline 3 & $A_{1} \times A_{1}$ & $\alpha+\beta$ & $\varnothing$ & \\
\hline 4 & $\mathrm{~A}_{r}$ & $\alpha_{1}+\alpha_{2}+\ldots+\alpha_{r}$ & $\begin{array}{c}\varnothing \text { for } r=2 ; \\
\alpha_{2}, \alpha_{3}, \ldots, \alpha_{r-1} \\
\text { for } r \geq 3\end{array}$ & $r \geq 2$ \\
\hline 5 & $A_{3}$ & $\alpha_{1}+2 \alpha_{2}+\alpha_{3}$ & $\alpha_{1}, \alpha_{3}$ & \\
\hline 6 & $\mathrm{~B}_{r}$ & $\alpha_{1}+\alpha_{2}+\ldots+\alpha_{r}$ & $\begin{array}{c}\varnothing \text { for } r=2 ; \\
\alpha_{2}, \alpha_{3}, \ldots, \alpha_{r-1} \\
\text { for } r \geq 3\end{array}$ & $r \geq 2$ \\
\hline 7 & $\mathrm{~B}_{r}$ & $2 \alpha_{1}+2 \alpha_{2}+\ldots+2 \alpha_{r}$ & $\alpha_{2}, \alpha_{3}, \ldots, \alpha_{r}$ & $r \geq 2$ \\
\hline 8 & $\mathrm{~B}_{3}$ & $\alpha_{1}+2 \alpha_{2}+3 \alpha_{3}$ & $\alpha_{1}, \alpha_{2}$ & \\
\hline 9 & $\mathrm{C}_{r}$ & $\alpha_{1}+2 \alpha_{2}+2 \alpha_{3}+\ldots+2 \alpha_{r-1}+\alpha_{r}$ & $\alpha_{3}, \alpha_{4}, \ldots, \alpha_{r}$ & $r \geq 3$ \\
\hline 10 & $\mathrm{D}_{r}$ & $2 \alpha_{1}+2 \alpha_{2}+\ldots+2 \alpha_{r-2}+\alpha_{r-1}+\alpha_{r}$ & $\alpha_{2}, \alpha_{3}, \ldots, \alpha_{r}$ & $r \geq 4$ \\
\hline 11 & $\mathrm{~F}_{4}$ & $\alpha_{1}+2 \alpha_{2}+3 \alpha_{3}+2 \alpha_{4}$ & $\alpha_{1}, \alpha_{2}, \alpha_{3}$ & \\
\hline 12 & $\mathrm{G}_{2}$ & $\alpha_{1}+\alpha_{2}$ & $\varnothing$ & \\
\hline 13 & $\mathrm{G}_{2}$ & $4 \alpha_{1}+2 \alpha_{2}$ & $\alpha_{2}$ & \\
\hline
\end{tabular}

Each element $\sigma \in \bar{\Sigma}(G)$ comes together with a certain subset $\Pi_{\sigma} \subset \operatorname{Supp} \sigma$, which can be defined as follows:

$$
\Pi_{\sigma}=\left\{\gamma \in \operatorname{Supp} \sigma \cap \sigma^{\perp} \mid \sigma-\gamma \notin \Delta^{+} \text {or } \gamma \in \operatorname{Supp}(\sigma-\gamma)\right\} .
$$

For the reader's convenience, in Table 1 we listed all roots in $\Pi_{\sigma}$ for each $\sigma \in \bar{\Sigma}(G)$. We note that

- $\Pi_{\sigma}=\operatorname{Supp} \sigma \cap \sigma^{\perp}$ unless $\sigma$ is in rows 6 or 9 of Table 1,

- $\Pi_{\sigma}=\left\{\gamma \in \operatorname{Supp} \sigma \cap \sigma^{\perp} \mid \sigma-\gamma \notin \Delta^{+}\right\}$unless $\sigma$ is in row 11 of Table 1 .

Observe that the set $\bar{\Sigma}(G)$ is finite and depends only on $G$.

We set

$$
\Phi(\Gamma)=\left\{\sigma \in \mathfrak{X}\left(T_{\mathrm{ad}}\right) \mid-\sigma \text { is a } T_{\mathrm{ad}} \text {-weight of } T_{X_{0}} \mathrm{M}_{\Gamma}\right\} .
$$

In other words, $\Phi(\Gamma)$ is the set of $T_{\mathrm{ad}}$-weights in the cotangent space of $\mathrm{M}_{\Gamma}$ at $X_{0}$.

Note that $0 \notin \Phi(\Gamma)$ by Corollary 2.31,

Theorem 3.1. The tangent space $T_{X_{0}} \mathrm{M}_{\Gamma}$ is a multiplicity-free $T_{\mathrm{ad}}$-module. Moreover, an element $\sigma \in \mathfrak{X}\left(T_{\mathrm{ad}}\right)$ belongs to $\Phi(\Gamma)$ if and only if the following conditions are satisfied:

(Ф1) $\sigma \in \mathbb{Z} \Gamma$

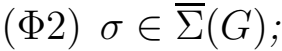

(Ф3) $\Pi_{\sigma} \subset \Gamma^{\perp}$; 
(\$4) if $\sigma=\alpha_{1}+\ldots+\alpha_{r}$ with $\operatorname{Supp} \sigma$ of type $\mathrm{B}_{r}(r \geq 2)$, then $\alpha_{r} \notin \Gamma^{\perp}$;

(Ф5) if $\sigma=\alpha+\beta$ with $\alpha, \beta \in \Pi$ and $\alpha \perp \beta$, then $\left\langle\alpha^{\vee}, \lambda\right\rangle=\left\langle\beta^{\vee}, \lambda\right\rangle$ for all $\lambda \in \Gamma$;

(Ф6) if $\sigma=2 \alpha$ for some $\alpha \in \Pi$ then $\left\langle\alpha^{\vee}, \lambda\right\rangle \in 2 \mathbb{Z}$ for all $\lambda \in \Gamma$;

(Ф7) if $\sigma \notin \Pi$ then for every $\varrho \in \mathcal{K}^{1}(\sigma)$ there exists $\delta \in \Pi \backslash \Gamma^{\perp}$ such that $\iota\left(\delta^{\vee}\right)$ is a positive multiple of $\varrho$;

(Ф8) if $\sigma=\alpha \in \Pi$ then there exist two distinct elements $\varrho_{1}, \varrho_{2} \in \mathcal{K} \cap \mathcal{L}$ with the following properties:

(a) $\left\langle\varrho_{1}, \alpha\right\rangle=\left\langle\varrho_{2}, \alpha\right\rangle=1$;

(b) $\iota\left(\alpha^{\vee}\right)=b_{1} \varrho_{1}+b_{2} \varrho_{2}$ for some $b_{1}, b_{2} \in \mathbb{Q}^{+} \backslash\{0\}$;

(c) $\mathcal{K}^{1}(\alpha) \subset\left\{\varrho_{1}, \varrho_{2}\right\}$.

Remark 3.2. In condition ( $(\Phi 8)$ it is important that the elements $\varrho_{1}, \varrho_{2}$ be distinct.

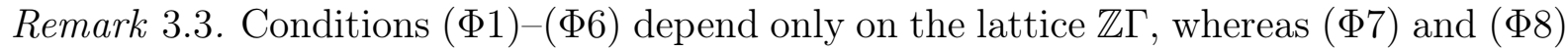
are the only conditions involving the cone $\mathbb{Q}^{+} \Gamma \subset \mathbb{Z} \Gamma \otimes_{\mathbb{Z}} \mathbb{Q}$.

Remark 3.4. In the case where $\Gamma$ is free, a result similar to Theorem 3.1 is proved by Bravi and Van Steirteghem in [BVS16]; see Theorem 4.1, Corollary 4.2, and Corollary 2.17 in loc. cit. Although our proof and that of loc. cit. follow the same general strategy, below we point out two main differences between the two approaches.

(1) When proving ( $\Phi 2)$ for every $\sigma \in \Phi(\Gamma)$, Bravi and Van Steirteghem establish a more general fact that (\$2) holds for any nonzero weight of the $T_{\text {ad }}$-module $\left(V / \mathfrak{g} x_{0}\right)^{G_{x_{0}}}$. However, their arguments resort to an extensive case-by-case analysis of root systems. On the other hand, in our proof of (\$2) for $\sigma \in \Phi(\Gamma)$ we avoid long case-by-case considerations thanks to Proposition 3.24 , which imposes strong restrictions on an element in $\left(\mathrm{V} / \mathfrak{g} x_{0}\right)^{G_{x_{0}}}$

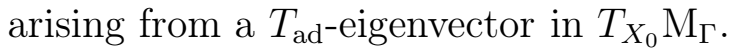

(2) To prove that every $\sigma \in \mathfrak{X}\left(T_{\mathrm{ad}}\right)$ satisfying $(\Phi 1)-(\Phi 8)$ belongs to $\Phi(\Gamma)$ (for free $\Gamma$ ), Bravi and Van Steirteghem use Theorem 2.23 and existence results for affine spherical $G$-varieties $X$ with $\Gamma_{X}=\Gamma$ and $\left|\Sigma_{X}\right|=1$, which trace back to the known classification of so-called wonderful varieties of rank 1 , see loc. cit. for details. In our proof, for every

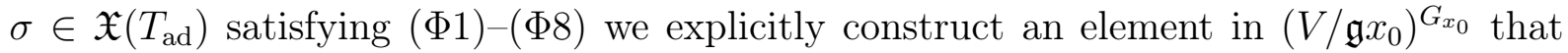
gives rise to a $T_{\text {ad }}$-eigenvector in $T_{X_{0}} \mathrm{M}_{\Gamma}$ of weight $-\sigma$.

Remark 3.5. In [BVS16], elements of $\bar{\Sigma}(G)$ are referred to as spherically closed spherical roots of $G$.

We now briefly describe the contents of the remaining part of this section. In $\S 3.2$ we gather further notation and conventions needed for the proof of Theorem [3.1. In $\S \S 3.3$ 3.6 we discuss several ingredients for the proof. The proof itself is divided into two steps carried out in $\S 3.7$ and $\S\left[3.8\right.$, respectively. At the first step we prove that the $T_{\text {ad }}$-module

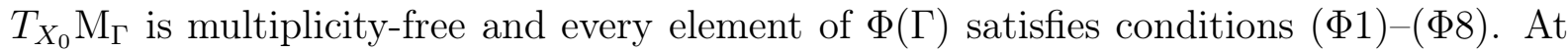

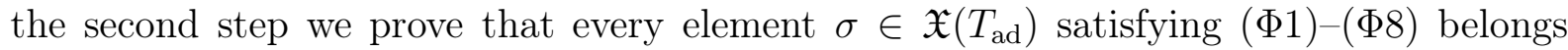
to $\Phi(\Gamma)$.

3.2. Preliminaries for the proof of Theorem 3.1. In this subsection we set up an additional notation and make several conventions that will be used in our proof of Theorem 3.1 .

We fix an arbitrary finite generating system $\mathrm{E} \subset \Gamma$. For every $\varrho \in \mathcal{K}^{1}$, we set

$$
\mathrm{E}_{\varrho}=\{\lambda \in \mathrm{E} \mid\langle\varrho, \lambda\rangle=0\} .
$$


Next, for every $\lambda \in \mathrm{E}$ we fix a lowest weight vector $v_{\lambda} \in V(\lambda)^{*}$ and put

$$
V=\bigoplus_{\lambda \in \mathrm{E}} V(\lambda)^{*}, \quad x_{0}=\sum_{\lambda \in \mathrm{E}} v_{\lambda}, \quad O=G x_{0}, \quad \text { and } \quad X_{0}=\bar{O} \subset V .
$$

From $\S 2.8$, recall the group $\widetilde{G}=G \lambda T_{\mathrm{ad}}$ and the action $a_{G}^{*}\left(\right.$ resp. $\left.a_{\mathrm{ad}}^{*}, a^{*}\right)$ of $G$ (resp. $\left.T_{\text {ad }}, \widetilde{G}\right)$ on $V$ under which $X_{0}$ is stable. Combining Propositions 2.28 and 2.29 we get a diagram of $T_{\text {ad }}$-equivariant maps

$$
\begin{aligned}
0 \rightarrow H^{0}\left(X_{0}, \mathcal{N}_{X_{0}}\right)^{\widetilde{G}} & H^{0}\left(X_{0}, \mathcal{N}_{X_{0}}\right)^{G} \rightarrow T_{X_{0}} \mathrm{M}_{\Gamma} \rightarrow 0 \\
& \left(V / \mathfrak{g} x_{0}\right)^{G_{x_{0}}}
\end{aligned}
$$

where the upper row is exact and the vertical arrow is an injective map. We identify $T_{X_{0}} \mathrm{M}_{\Gamma}$ with the unique $T_{\text {ad }}$-submodule of $H^{0}\left(X_{0}, \mathcal{N}_{X_{0}}\right)^{G}$ complementary to $H^{0}\left(X_{0}, \mathcal{N}_{X_{0}}\right)^{\widetilde{G}}$ and let $\mathcal{T S}$ denote the image of $T_{X_{0}} \mathrm{M}_{\Gamma}$ in $\left(V / \mathfrak{g} x_{0}\right)^{G_{x_{0}}}$, so that

$$
\mathcal{T S} \simeq T_{X_{0}} \mathrm{M}_{\Gamma}
$$

as $T_{\text {ad }}$-modules.

For our computations with the $T_{\text {ad }}$-module $\left(V / \mathfrak{g} x_{0}\right)^{G_{x_{0}}}$, it will be more convenient to replace the actions $a_{G}^{*}, a_{\text {ad }}^{*}$, and $a^{*}$ with other ones as described below.

As in $\S 2.8$, we let $\bar{t}$ denote the image in $T_{\text {ad }}$ of an element $t \in T$. Let $\theta \in$ Aut $G$ be a Weyl involution of $G$ relative to $T$, that is, $\theta(t)=t^{-1}$ for all $t \in T$. It is well known that $\theta(B) \cap B=T$. We extend this involution to an involution of $\widetilde{G}$ by setting $\theta(\bar{t})=\bar{t}^{-1}$ for all $\bar{t} \in T_{\mathrm{ad}}$.

We define a new action $a:((g, \bar{t}), v) \mapsto(g, \bar{t}) \cdot v$ of $\widetilde{G}$ on $V$ by $(g, \bar{t}) \cdot v=\theta(g, \bar{t}) * v$. Let $a_{G}\left(\right.$ resp. $\left.a_{\text {ad }}\right)$ denote the restriction of $a$ to $G$ (resp. $\left.T_{\text {ad }}\right)$.

Here are the most important features of the new actions.

(1) The action $a_{\mathrm{ad}}$ is opposite to the action $a_{\mathrm{ad}}^{*}$. In particular, the set $\Phi(\Gamma)$ is exactly the set of weights of the $T_{\text {ad }}$-module $\mathcal{T S}$ with respect to the action $a_{\text {ad }}$.

(2) For every $\lambda \in \mathrm{E}$, the subspace $V(\lambda)^{*} \subset V$, regarded as a $G$-module with respect to the action $a_{G}$, is isomorphic to $V(\lambda)$ with $v_{\lambda}$, viewed in $V(\lambda)$, being a highest weight vector.

From now on, we shall consider the diagram (3.3) only with respect to the actions $a$, $a_{G}$, and $a_{\text {ad }}$. According to (2), this implies the following changes in our notation:

- $V=\bigoplus_{\lambda \in \mathrm{E}} V(\lambda)$

- $v_{\lambda}$ is a highest weight vector of $V(\lambda)$ for every $\lambda \in$ E.

With the above new notation, $a_{G}$ becomes the usual action of $G$ on $V$ and the action $a_{\text {ad }}$ of $T_{\mathrm{ad}}$ on $V$ is given by

$$
\bar{t} \cdot v=t^{\lambda}\left(t^{-1} \cdot v\right) \text { for all } t \in T, \lambda \in \mathrm{E} \text {, and } v \in V(\lambda) \text {. }
$$

For every $\lambda \in \mathrm{E}$, let $\mathrm{p}_{\lambda}: V \rightarrow V(\lambda)$ be the canonical projection.

For every $v \in V$, let $[v]$ denote the image of $v$ under the natural map $V \rightarrow V / \mathfrak{g} x_{0}$.

Since the subspace $\mathfrak{g} x_{0} \subset V$ is $T_{\text {ad }}$-invariant, for every $T_{\text {ad }}$-eigenvector $q \in V / \mathfrak{g} x_{0}$ there exists a $T_{\text {ad }}$-eigenvector $v \in V$ (of the same weight) such that $[v]=q$. This observation will be always used in our study of $\left(V / \mathfrak{g} x_{0}\right)^{G_{x_{0}}}$. 
3.3. The role of saturatedness of $\Gamma$. The saturatedness assumption on $\Gamma$ will be essential in our proof of Theorem 3.1. Firstly, by Proposition 2.11 this assumption guarantees that the variety $X_{0}$ is normal, which is essentially used in the proof of Proposition 3.13 in $\$ 3.5$. Secondly, our arguments will often require the following crucial property of saturated $\Gamma$.

Lemma 3.6. For every $\varrho \in \mathcal{K}^{1}$, there exists $\mu \in \mathrm{E}$ such that $\langle\varrho, \mu\rangle=1$.

Proof. It suffices to prove that $\{\nu \in \Gamma \mid\langle\varrho, \nu\rangle=1\} \neq \varnothing$. Since $\Gamma$ is saturated, one has

$$
\Gamma=\left\{\nu \in \mathbb{Z} \Gamma \mid\langle\kappa, \nu\rangle \geq 0 \text { for all } \kappa \in \mathcal{K}^{1}\right\} .
$$

As $\varrho$ is primitive in $\mathcal{L}$, there exists $\nu_{0} \in \mathbb{Z} \Gamma$ with $\left\langle\varrho, \nu_{0}\right\rangle=1$. If $\mathcal{K}^{1}=\{\varrho\}$ then $\nu_{0} \in \Gamma$ by (3.5). Otherwise there exists an element $\eta \in \mathbb{Z}^{+} \mathrm{E}_{\varrho}$ such that $\langle\kappa, \eta\rangle>0$ for all $\kappa \in \mathcal{K}^{1} \backslash\{\varrho\}$. For each $n \in \mathbb{Z}^{+}$consider the element $\nu_{n}=\nu_{0}+n \eta$. Clearly, $\left\langle\varrho, \nu_{n}\right\rangle=1$ for all $n \in \mathbb{Z}^{+}$. In view of (3.5) one has $\nu_{n} \in \Gamma$ when $n$ is sufficiently large.

3.4. Basic properties of $\left(V / \mathfrak{g} x_{0}\right)^{G_{x_{0}}}$ and its $T_{\text {ad }}$-weights. The material presented in this subsection is more or less known.

The following lemma is obvious.

Lemma 3.7. Suppose that $\mathrm{E}^{\prime} \subset \mathrm{E}$ is a nonempty subset, $x=\sum_{\lambda \in \mathrm{E}^{\prime}} v_{\lambda} \in V$, and $A$ is a $G_{x}$-module. Then an element $a \in A$ is $G_{x}$-fixed if and only if the following two conditions hold:

(1) a is $T_{x}$-stable;

(2) $e_{\delta} a=0$ for all $\delta \in \Delta^{+} \cup\left(\Delta^{-} \cap \mathbb{Z} \mathrm{E}^{\prime \perp}\right)$.

Moreover, condition (2) is equivalent to

$\left(2^{\prime}\right) e_{\delta} a=0$ for all $\delta \in \Pi \cup\left(-\mathrm{E}^{\prime \perp}\right)$.

Lemma 3.8. Suppose that $\sigma$ is a $T_{\mathrm{ad}}$-weight of $\left(V / \mathfrak{g} x_{0}\right)^{G_{x_{0}}}$. Then

(a) $\sigma \in \mathbb{Z}^{+} \Pi$;

(b) $\sigma \in \mathbb{Z} \Gamma$.

Proof. (国) This follows from (3.4) and basic properties of $T$-weights in a simple $G$-module.

(b) It suffices to show that $t^{\sigma}=1$ for all $t \in T_{x_{0}}$. Assume the converse and take $t \in T_{x_{0}}$

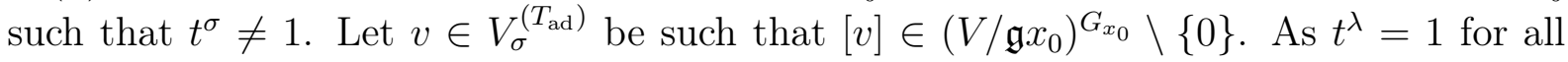
$\lambda \in \mathrm{E}$, one has $t \cdot v=t^{-\sigma} v$. Since $T_{x_{0}} \subset G_{x_{0}}$, it follows that $[v]=[t \cdot v]=t^{-\sigma}[v]$ and hence $[v]=0$, a contradiction.

Lemma 3.9. Suppose that $\sigma$ is a nonzero $T_{\mathrm{ad}}$-weight of $V$ and $v \in V_{\sigma}^{\left(T_{\mathrm{ad}}\right)} \backslash\{0\}$. Then there exists $\delta \in \Pi$ such that $e_{\delta} v \neq 0$.

Proof. Assume that $e_{\delta} v=0$ for all $\delta \in \Pi$. Then $v$ is a sum of highest weight vectors in $V$. As $\sigma \neq 0$, it follows that $v=0$, a contradiction.

Lemma 3.10. Let $\sigma$ be a $T_{\mathrm{ad}}$-weight of $\left(V / \mathfrak{g} x_{0}\right)^{G_{x_{0}}}$. Suppose that $v \in V_{\sigma}^{\left(T_{\mathrm{ad}}\right)}$ is such that $[v] \in\left(V / \mathfrak{g} x_{0}\right)^{G_{x_{0}}}$. Then $e_{\delta} v \in V_{\sigma-\delta}^{\left(T_{\mathrm{ad}}\right)} \cap \mathfrak{g} x_{0}$ for every $\delta \in \Delta^{+} \cup\left(\Delta^{-} \cap \mathbb{Z} \mathrm{E}^{\perp}\right)$.

Proof. By Lemma 3.7 the condition $\delta \in \Delta^{+} \cup\left(\Delta^{-} \cap \mathbb{Z E}^{\perp}\right)$ implies $e_{\delta} v \in \mathfrak{g} x_{0}$. Clearly, $e_{\delta} v$

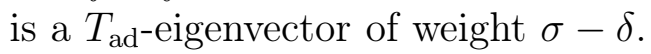


Lemma 3.11. Suppose that $\sigma \in \mathbb{Z} \Pi$. Then

$$
V_{\sigma}^{\left(T_{\text {ad }}\right)} \cap \mathfrak{g} x_{0}= \begin{cases}\mathbb{k} e_{-\sigma} x_{0} & \text { if } \sigma \in \Delta^{+} \\ \mathfrak{t} x_{0} & \text { if } \sigma=0 ; \\ \{0\} & \text { otherwise. }\end{cases}
$$

Proof. This follows from the decomposition $\mathfrak{g}=\mathfrak{t} \oplus \bigoplus_{\delta \in \Delta} \mathbb{k} e_{\delta}$ and the fact that $e_{\delta} x_{0}=0$ for all $\delta \in \Delta^{+}$.

Corollary 3.12. Let $\sigma$ be a nonzero $T_{\mathrm{ad}}$-weight of $\left(V / \mathfrak{g} x_{0}\right)^{G_{x_{0}}}$. Let $v \in V_{\sigma}^{\left(T_{\mathrm{ad}}\right)}$ be such that $[v] \in\left(V / \mathfrak{g} x_{0}\right)^{G_{x_{0}}} \backslash\{0\}$. Suppose that $\delta \in \Delta^{+}$is such that $\delta \neq \sigma$ and $e_{\delta} v \neq 0$. Then

(a) $\sigma-\delta \in \Delta^{+}$;

(b) $e_{\delta} v=c e_{-(\sigma-\delta)} x_{0}$ for some $c \in \mathbb{k}^{\times}$.

Proof. This is a direct consequence of Lemmas 3.10 and 3.11 ,

3.5. Extension of sections. For every open subset $Y \subset X_{0}$, we let $\mathcal{N}_{Y}$ denote the restriction of the sheaf $\mathcal{N}_{X_{0}}$ to $Y$.

Proposition 3.13. For a section $s \in H^{0}\left(O, \mathcal{N}_{O}\right)$, the following conditions are equivalent:

(1) $s$ extends to $X_{0}$.

(2) s extends to $O \cup O^{\prime}$ for each $G$-orbit $O^{\prime} \subset X_{0}$ of codimension 1 .

Proof. Let $Y \subset X_{0}$ be the union of all $G$-orbits in $X_{0}$ of codimension at most 1 . Then $Y$ is an open $G$-stable subset of $X_{0}$ and $\operatorname{codim}_{X_{0}}\left(X_{0} \backslash Y\right) \geq 2$. As $X_{0}$ is normal, by Br13, Lemma 3.9] the restriction map $H^{0}\left(X_{0}, \mathcal{N}_{X_{0}}\right) \rightarrow H^{0}\left(Y, \mathcal{N}_{Y}\right)$ is an isomorphism. Thus a section $s \in H^{0}\left(O, \mathcal{N}_{O}\right)$ extends to $X_{0}$ if and only if it extends to $Y$. But the latter is obviously equivalent to (2).

To describe all $G$-orbits in $X_{0}$ of codimension 1, we need some additional notation. First of all, we introduce the set

$$
\mathcal{P}=\left\{\varrho \in \mathcal{K}^{1} \mid \mathrm{E}^{\perp}=\mathrm{E}_{\varrho}^{\perp}\right\} .
$$

Note that every $\varrho \in \mathcal{P}$ is not proportional to an element of the form $\iota\left(\alpha^{\vee}\right)$ with $\alpha \in \Pi$. Next, for every $\varrho \in \mathcal{P}$ we consider the vector

$$
z_{\varrho}=\sum_{\lambda \in \mathrm{E}_{\varrho}} v_{\lambda} \in V
$$

and its $G$-orbit $O_{\varrho}=G z_{\varrho}$.

The following result is a consequence of [ViP72, Theorems 8 and 9].

Proposition 3.14. The map $\varrho \mapsto O_{\varrho}$ is a bijection between the set $\mathcal{P}$ and the $G$-orbits in $X_{0}$ of codimension 1 .

Corollary 3.15. Suppose that $\sigma$ is a nonzero $T_{\mathrm{ad}}$-weight of $V$ and $v \in V_{\sigma}^{\left(T_{\mathrm{ad}}\right)}$. Let $s \in H^{0}\left(O, \mathcal{N}_{O}\right)^{G}$ be the section defined by $s\left(x_{0}\right)=[v]$. Then the following conditions are equivalent:

(1) $[v] \in \mathcal{T S}$.

(2) $s$ extends to $O \cup O_{\varrho}$ for all $\varrho \in \mathcal{P}$. 
Proof. This follows from the definition of $\mathcal{T S}$ (see $\S 3.2$ ) along with Propositions 3.13 and 3.14 .

In what follows, for every $\varrho \in \mathcal{P}$ we regard the sheaf $\mathcal{N}_{O \cup O_{\varrho}}$ as the sheaf of sections of the normal bundle of $O \cup O_{\varrho}$ in $V$. We denote the total space of this bundle by $F_{\varrho}$ and let

$$
p_{\varrho}: F_{\varrho} \rightarrow O \cup O_{\varrho}
$$

be the canonical projection.

Fix an arbitrary element $\varrho \in \mathcal{P}$ and let $\phi_{\varrho}: \mathbb{k}^{\times} \rightarrow T$ be the one-parameter subgroup of $T$ corresponding to $\varrho$, that is, $\left(\phi_{\varrho}(\xi)\right)^{\chi}=\xi^{\langle\varrho, \chi\rangle}$ for all $\chi \in \mathfrak{X}(T)$ and $\xi \in \mathbb{k}^{\times}$. For every $\xi \in \mathbb{k}$, consider the vector $z_{\varrho}(\xi) \in V$ given by

$$
z_{\varrho}(\xi)= \begin{cases}z_{\varrho} & \text { if } \xi=0 ; \\ \phi_{\varrho}(\xi) x_{0} & \text { otherwise. }\end{cases}
$$

Then we have $z_{\varrho}(\xi)=z_{\varrho}+\sum_{\lambda \in \mathrm{E} \backslash \mathrm{E}_{\varrho}} \xi^{\lfloor\varrho, \lambda\rangle} v_{\lambda}$ for all $\xi \in \mathbb{k}$. Note that $z_{\varrho}(1)=x_{0}$. It follows from Lemma 3.6 that the morphism $\mathbb{k} \rightarrow O \cup O_{\varrho}$ given by $\xi \mapsto z_{\varrho}(\xi)$ is a closed immersion; we denote its image by $Z_{\varrho}$.

Lemma 3.16. Suppose that $\varrho \in \mathcal{P}$. Then $T_{z_{\varrho}} X_{0}=\mathfrak{g} z_{\varrho} \oplus \mathbb{k} u_{\varrho}$ where

$$
u_{\varrho}=\sum_{\mu \in \mathrm{E}:\langle\varrho, \mu\rangle=1} v_{\mu} \text {. }
$$

Proof. We have $T_{z_{\varrho}}\left(G z_{\varrho}\right)=\mathfrak{g} z_{\varrho}$ and $T_{z_{\varrho}} Z_{\varrho}=\mathbb{k} u_{\varrho}$. Since $G z_{\varrho}$ has codimension 1 in $X_{0}$ and $X_{0}$ is normal, it follows that $\operatorname{dim} \mathfrak{g} z_{\varrho}=\operatorname{dim} X_{0}-1$ and $z_{\varrho}$ is a regular point of $X_{0}$. The proof is completed by observing that $u_{\varrho} \notin \mathfrak{g} z_{\varrho}$.

Proposition 3.17. Let $v \in V$ be such that $[v] \in\left(V / \mathfrak{g} x_{0}\right)^{G_{x_{0}}}$ and let $s \in H^{0}\left(O, \mathcal{N}_{O}\right)^{G}$ be the section defined by $s\left(x_{0}\right)=[v]$. Given $\varrho \in \mathcal{P}$, the following conditions are equivalent:

(1) The section $s$ extends to $O \cup O_{\varrho}$.

(2) There exists $\lim _{\xi \rightarrow 0} s\left(z_{\varrho}(\xi)\right)$, that is, the restriction of $s$ to $O \cap Z_{\varrho}$ extends to $Z_{\varrho}$.

Proof. Obviously, (11) implies (21). It remains to prove the converse implication. Put $s\left(z_{\varrho}\right)=\lim _{\xi \rightarrow 0} s\left(z_{\varrho}(\xi)\right)$. Being in the closure of the $\phi_{\varrho}\left(\mathbb{k}^{\times}\right)$-orbit of $s\left(x_{0}\right)$, the point $s\left(z_{\varrho}\right)$ is $\phi_{\varrho}\left(\mathbb{k}^{\times}\right)$-fixed. Further, observe that $G_{x_{0}}=G_{z_{\varrho}(\xi)}$ for all $\xi \in \mathbb{k}^{\times}$, which implies that every point $s\left(z_{\varrho}(\xi)\right)$ with $\xi \in \mathbb{R}^{\times}$is $G_{x_{0}}$-fixed. Consequently, the point $s\left(z_{\varrho}\right)$ is $G_{x_{0}}$-fixed. Next, as $\mathbb{Q}^{+} \mathrm{E}_{\varrho}$ is a face of codimension 1 of $\mathbb{Q}^{+} \Gamma$ and $\Gamma$ is saturated, it follows that $\mathbb{Z} \Gamma \cap \operatorname{Ker} \varrho=\mathbb{Z} \mathrm{E}_{\varrho}$, which implies $T_{z_{\varrho}}=T_{x_{0}} \cdot \phi_{\varrho}\left(\mathbb{k}^{\times}\right)$. Combining this and the equality $\mathrm{E}^{\perp}=\mathrm{E}_{\varrho}^{\perp}$ with Lemma 3.7 we obtain $G_{z_{\varrho}}=G_{x_{0}} \cdot \phi_{\varrho}\left(\mathbb{k}^{\times}\right)$, hence $s\left(z_{\varrho}\right)$ is $G_{z_{e}}$-fixed. The latter enables us to extend $s$ to $O_{\varrho}$ by the formula $s\left(g z_{\varrho}\right)=g\left(s\left(z_{\varrho}\right)\right)$.

To complete the proof it remains to show that the extended map $s: O \cup O_{\varrho} \rightarrow F_{\varrho}$ is a morphism. First, as $s\left(z_{\varrho}\right)=\lim _{\xi \rightarrow 0} s\left(z_{\varrho}(\xi)\right)$, it follows that the $G$-orbit $G s\left(z_{\varrho}\right)$ is contained in the closure of the $G$-orbit $G s\left(x_{0}\right)$ in $F_{\varrho}$. Next, since the map $p_{\varrho}$ is $G$-equivariant and the point $s\left(x_{0}\right)$ is $G_{x_{0}}$-fixed, it follows that $G_{s\left(x_{0}\right)}=G_{x_{0}}$ and hence $G s\left(x_{0}\right) \simeq O$. Similarly, $G s\left(z_{\varrho}\right) \simeq O_{\varrho}$. In particular, $\operatorname{dim} G s\left(z_{\varrho}\right)=\operatorname{dim} G s\left(x_{0}\right)-1$, and so the set $G s\left(x_{0}\right) \cup G s\left(z_{\varrho}\right)$ is open in $\overline{G s\left(x_{0}\right)}$. Now the restriction of $p_{\varrho}$ to $G s\left(x_{0}\right) \cup G s\left(z_{\varrho}\right)$ is a bijective morphism 
onto $O \cup O_{\varrho}$, whence an isomorphism since $O \cup O_{\varrho}$ is smooth. Consequently, our map $s: O \cup O_{\varrho} \rightarrow G s\left(x_{0}\right) \cup G s\left(z_{\varrho}\right)$ is a morphism as required.

The next proposition is an application of the previous one.

Proposition 3.18. Suppose that $\varrho \in \mathcal{P}$ and $\sigma \in \mathbb{Z}^{+} \Pi$. Let $v \in V_{\sigma}^{\left(T_{\text {ad }}\right)}$ be such that $[v] \in\left(V / \mathfrak{g} x_{0}\right)^{G_{x_{0}}} \backslash\{0\}$ and let $s \in H^{0}\left(O, \mathcal{N}_{O}\right)^{G}$ be the section defined by $s\left(x_{0}\right)=[v]$.

(a) If $\langle\varrho, \sigma\rangle>0$ and $\sum_{\lambda \in \mathrm{E}_{\varrho}} \mathrm{p}_{\lambda}(v) \notin \mathfrak{g} z_{\varrho}$, then $s$ does not extend to $O \cup O_{\varrho}$.

(b) If $\langle\varrho, \sigma\rangle \leq 0$ then $s$ extends to $O \cup O_{\varrho}$.

(c) If $\sum_{\lambda \in \mathrm{E}_{\varrho}} \mathrm{p}_{\lambda}(v)=0$ and there exists $\nu \in \mathrm{E} \backslash \mathrm{E}_{\varrho}$ with $\mathrm{p}_{\nu}(v) \neq 0$ and $\langle\varrho, \sigma\rangle>\langle\varrho, \nu\rangle$, then $s$ does not extend to $O \cup O_{\varrho}$.

(d) If $\sum_{\lambda \in \mathrm{E}_{\varrho}} \mathrm{p}_{\lambda}(v)=0$ and $\langle\varrho, \sigma\rangle=1$ then $s$ extends to $O \cup O_{\varrho}$.

Proof. Thanks to Proposition 3.17, in all the cases it is enough to prove the corresponding statement about the existence of $\lim _{\xi \rightarrow 0} s\left(z_{\varrho}(\xi)\right)$. Before we proceed, let us make some preparations.

Given $w \in V_{\sigma}^{\left(T_{\text {ad }}\right)}$ and $\xi \in \mathbb{k}$, let $[w]_{\xi}$ denote the image of $w$ in $V / T_{z_{\varrho}(\xi)} X_{0}$. Then for every $\xi \in \mathbb{k}^{\times}$one has

$$
s\left(z_{\varrho}(\xi)\right)=s\left(\phi_{\varrho}(\xi) x_{0}\right)=\xi^{-\langle\varrho, \sigma\rangle}\left[\sum_{\lambda \in \mathrm{E}_{\varrho}} \mathrm{p}_{\lambda}(v)\right]_{\xi}+\sum_{\mu \in \mathrm{E} \backslash \mathrm{E}_{\varrho}} \xi^{\langle\varrho, \mu\rangle-\langle\varrho, \sigma\rangle}\left[\mathrm{p}_{\mu}(v)\right]_{\xi} .
$$

Let $\mathcal{N}_{Z_{\varrho}}$ denote the restriction of the sheaf $\mathcal{N}_{O \cup O_{\varrho}}$ to $Z_{\varrho}$.

(国) Consider the section $s^{\prime} \in H^{0}\left(Z_{\varrho}, \mathcal{N}_{Z_{\varrho}}\right)$ given by

$$
s^{\prime}\left(z_{\varrho}(\xi)\right)=\left[\sum_{\lambda \in \mathrm{E}_{\varrho}} \mathrm{p}_{\lambda}(v)\right]_{\xi}+\sum_{\mu \in \mathrm{E} \backslash \mathrm{E}_{\varrho}} \xi^{\langle\varrho, \mu\rangle}\left[\mathrm{p}_{\mu}(v)\right]_{\xi} .
$$

Clearly, $s^{\prime}\left(z_{\varrho}\right)=\left[\sum_{\lambda \in \mathrm{E}_{\varrho}} \mathrm{p}_{\lambda}(v)\right]_{0}$. It follows from Lemma 3.16 that $\left(\bigoplus_{\lambda \in \mathrm{E}_{\varrho}} V(\lambda)\right) \cap T_{z_{\varrho}} X_{0}=\mathfrak{g} z_{\varrho}$, whence the condition $\sum_{\lambda \in \mathrm{E}_{\varrho}} \mathrm{p}_{\lambda}(v) \notin \mathfrak{g} z_{\varrho}$ implies $s^{\prime}\left(z_{\varrho}\right) \neq 0$. On the other hand, one has $s\left(z_{\varrho}(\xi)\right)=\xi^{-\langle\varrho, \sigma\rangle} s^{\prime}\left(z_{\varrho}(\xi)\right)$ for all $\xi \in \mathbb{k}^{\times}$. Since $\langle\varrho, \sigma\rangle>0$, it follows that $\lim _{\xi \rightarrow 0} s\left(z_{\varrho}(\xi)\right)$ does not exist.

(b) It is easy to see that $\lim _{\xi \rightarrow 0} s\left(z_{\varrho}(\xi)\right)$ exists and is given by

$$
\lim _{\xi \rightarrow 0} s\left(z_{\varrho}(\xi)\right)= \begin{cases}\sum_{\lambda \in \mathrm{E}_{\varrho}}\left[\mathrm{p}_{\lambda}(v)\right]_{0} & \text { if }\langle\varrho, \sigma\rangle=0 \\ 0 & \text { if }\langle\varrho, \sigma\rangle<0\end{cases}
$$

(ㄷ) We may assume that $\langle\varrho, \nu\rangle \leq\langle\varrho, \mu\rangle$ for all $\mu \in \mathrm{E} \backslash \mathrm{E}_{\varrho}$ with $\mathrm{p}_{\mu}(v) \neq 0$. Consider the section $s^{\prime} \in H^{0}\left(Z_{\varrho}, \mathcal{N}_{Z_{\varrho}}\right)$ given by

$$
s^{\prime}\left(z_{\varrho}(\xi)\right)=\sum_{\mu \in \mathrm{E} \backslash \mathrm{E}_{\varrho}:\langle\varrho, \mu\rangle=\langle\varrho, \nu\rangle}\left[\mathrm{p}_{\mu}(v)\right]_{\xi}+\sum_{\mu \in \mathrm{E} \backslash \mathrm{E}_{\varrho}:\langle\varrho, \mu\rangle>\langle\varrho, \nu\rangle} \xi^{\langle\varrho, \mu\rangle-\langle\varrho, \nu\rangle}\left[\mathrm{p}_{\mu}(v)\right]_{\xi} .
$$


Clearly, $s^{\prime}\left(z_{\varrho}\right)=\sum_{\mu \in \mathrm{E} \backslash \mathrm{E}_{\varrho}:\langle\varrho, \mu\rangle=\langle\varrho, \nu\rangle}\left[\mathrm{p}_{\mu}(v)\right]_{0}$. Since $\langle\varrho, \sigma\rangle>\langle\varrho, \nu\rangle>0$, it follows that $\sigma \neq 0$ and hence

$$
\sum_{\mu \in \mathrm{E} \backslash \mathrm{E}_{\varrho}:\langle\varrho, \mu\rangle=\langle\varrho, \nu\rangle} \mathrm{p}_{\mu}(v) \notin \mathbb{k} u_{\varrho} .
$$

As $\mathbb{k} u_{\varrho}=\left(\bigoplus_{\mu \in \mathrm{E} \backslash \mathrm{E}_{\varrho}} V(\mu)\right) \cap T_{z_{\varrho}} X_{0}$ by Lemma 3.16, we find that $s^{\prime}\left(z_{\varrho}\right) \neq 0$. On the other hand, we have $s\left(z_{\varrho}(\xi)\right)=\xi^{\langle\varrho, \nu\rangle-\langle\varrho, \sigma\rangle} s^{\prime}\left(z_{\varrho}(\xi)\right)$ for all $\xi \in \mathbb{k}^{\times}$. Since $\langle\varrho, \nu\rangle<\langle\varrho, \sigma\rangle$, it follows that $\lim _{\xi \rightarrow 0} s\left(z_{\varrho}(\xi)\right)$ does not exist.

(d) Clearly, $\lim _{\xi \rightarrow 0} s\left(z_{\varrho}(\xi)\right)$ exists and equals $\sum_{\mu \in \mathrm{E}:\langle\varrho, \mu\rangle=1}\left[\mathrm{p}_{\mu}(v)\right]_{0}$.

Remark 3.19. In [PVS16, Theorem 2.8] one can find a specialization of our Proposition 3.18 to the case where $\Gamma$ is free.

3.6. Canonical representatives of $T_{\text {ad }}$-eigenvectors in $\mathcal{T S}$. The main results of this subsection are Propositions 3.24 and 3.26 .

Lemma 3.20. For every $\sigma \in \mathbb{Z}^{+} \Pi \backslash\{0\}$ there exists $\delta \in \operatorname{Supp} \sigma$ such that $\left\langle\delta^{\vee}, \sigma\right\rangle>0$.

Proof. Assuming the converse we find that the angle between any two distinct elements of the set $\{\sigma\} \cup \operatorname{Supp} \sigma$ is non-acute. Since the latter set is contained in a half-space of the $\mathbb{Q}$-vector space spanned by $\operatorname{Supp} \sigma$, the elements in $\{\sigma\} \cup \operatorname{Supp} \sigma$ have to be linearly independent, which is not the case.

Recall from (3.1) the set $\mathcal{K}^{1}(\sigma) \subset \mathcal{K}^{1}$ defined for every $\sigma \in \mathbb{Z} \Gamma$. As $\Phi(\Gamma) \subset \mathbb{Z} \Gamma$ by Lemma $3.8(\mathrm{~b})$, the set $\mathcal{K}^{1}(\sigma)$ is also defined for every $\sigma \in \Phi(\Gamma)$.

Lemma 3.21. Suppose that $\sigma \in \mathbb{Z} \Gamma \cap\left(\mathbb{Z}^{+} \Pi \backslash\{0\}\right)$. Then $\mathcal{K}^{1}(\sigma) \neq \varnothing$.

Proof. By Lemma 3.20 there exists $\delta \in \Pi$ with $\left\langle\delta^{\vee}, \sigma\right\rangle>0$. Now assume $\mathcal{K}^{1}(\sigma)=\varnothing$. Then $\langle\varrho, \sigma\rangle \leq 0$ for all $\varrho \in \mathcal{K}^{1}$, hence $\sigma \in-\Gamma$. The latter yields $\left\langle\delta^{\vee}, \sigma\right\rangle \leq 0$, a contradiction.

Corollary 3.22. Suppose that $\sigma \in \Phi(\Gamma)$. Then $\mathcal{K}^{1}(\sigma) \neq \varnothing$.

Proof. This follows from Corollary 2.31 along with Lemmas 3.8 and 3.21.

Recall the subset $\mathcal{P} \subset \mathcal{K}^{1}$ given by (3.6).

Lemma 3.23. Suppose that $\varrho \in \mathcal{K}^{1} \backslash \mathcal{P}$. Then there exists $\delta \in \Pi$ such that $\iota\left(\delta^{\vee}\right)$ is a positive multiple of $\varrho$.

Proof. Since $\varrho \notin \mathcal{P}$, it follows that $\mathrm{E}^{\perp} \neq \mathrm{E}_{\varrho}^{\perp}$. Then there exist $\delta \in \Pi$ and $\mu \in \mathrm{E} \backslash \mathrm{E}_{\varrho}$ such that $\left\langle\delta^{\vee}, \mu\right\rangle>0$ and $\left\langle\delta^{\vee}, \lambda\right\rangle=0$ for all $\lambda \in \mathrm{E}_{\varrho}$. Obviously, $\delta$ possesses the required property.

Proposition 3.24. Let $\sigma \in \Phi(\Gamma), \varrho \in \mathcal{K}^{1}(\sigma), v \in V_{\sigma}^{\left(T_{\mathrm{ad}}\right)}$, and $[v] \in \mathcal{T S} \backslash\{0\}$.

(a) If $\sigma \in \Delta^{+}$then there exist $v^{\prime} \in V_{\sigma}^{\left(T_{\text {ad }}\right)}$ and $c \in \mathbb{k}$ such that $v^{\prime}=v-c e_{-\sigma} x_{0}$ and $\mathrm{p}_{\lambda}\left(v^{\prime}\right)=0$ for all $\lambda \in \mathrm{E}_{\varrho}$.

(b) If $\sigma \notin \Delta^{+}$then $\mathrm{p}_{\lambda}(v)=0$ for all $\lambda \in \mathrm{E}_{\varrho}$. 
Proof. Note that $\sigma \neq 0$ by Corollary 2.31. Recall the vector $z_{\varrho}$ given by (3.7) and set $w=\sum_{\lambda \in \mathrm{E}_{\varrho}} \mathrm{p}_{\lambda}(v)$. We consider two cases.

Case 1: $\varrho \in \mathcal{P}$. Since $[v] \in \mathcal{T S}$, it follows from Corollary 3.15 and Proposition 3.18 (国) that $w \in \mathfrak{g} z_{\varrho}$. Applying an analogue of Lemma 3.11 for $V_{\sigma}^{\left(T_{\text {ad }}\right)} \cap \mathfrak{g} z_{\varrho}$, we obtain the following:

- if $\sigma \notin \Delta^{+}$then $w=0$;

- if $\sigma \in \Delta^{+}$then $w=c e_{-\sigma} z_{\varrho}$ for some $c \in \mathbb{k}$.

In the latter case, the vector $v^{\prime}=v-c e_{-\sigma} x_{0}$ satisfies $\mathrm{p}_{\lambda}\left(v^{\prime}\right)=0$ for all $\lambda \in \mathrm{E}_{\varrho}$.

Case 2: $\varrho \notin \mathcal{P}$. Assume that $w \neq 0$. By Lemma 3.23, there exists $\delta \in \Pi$ such that $\iota\left(\delta^{\vee}\right)$ is a positive multiple of $\varrho$. Then $\left\langle\delta^{\vee}, \sigma\right\rangle>0$ and $\left\langle\delta^{\vee}, \lambda\right\rangle=0$ for all $\lambda \in \mathrm{E}_{\varrho}$. If $\sigma=\delta$ then $\mathrm{p}_{\lambda}(v) \in \mathbb{k} e_{-\delta} v_{\lambda}=\{0\}$ for every $\lambda \in \mathrm{E}_{\varrho}$, which contradicts the assumption $w \neq 0$. So in what follows we assume that $\sigma \neq \delta$. We have $\left\langle\delta^{\vee}, \lambda-\sigma\right\rangle=-\left\langle\delta^{\vee}, \sigma\right\rangle<0$ for all $\lambda \in \mathrm{E}_{\varrho}$, therefore $e_{\delta} w \neq 0$ and hence $e_{\delta} v \neq 0$. Corollary 3.12 implies that $\sigma-\delta \in \Delta^{+}$and $e_{\delta} v=c e_{-(\sigma-\delta)} x_{0}$ for some $c \in \mathbb{k}^{\times}$. In particular, $e_{\delta} \mathrm{p}_{\lambda}(v)=c e_{-(\sigma-\delta)} v_{\lambda}$ for all $\lambda \in \mathrm{E}$.

Let $\mathfrak{h} \simeq \mathfrak{s l}_{2}$ be the Lie subalgebra of $\mathfrak{g}$ generated by $e_{\delta}$ and $e_{-\delta}$. Fix $\lambda \in \mathrm{E}_{\varrho}$ such that $\mathrm{p}_{\lambda}(v) \neq 0$. Let $R^{\lambda} \subset V(\lambda)$ be the $\mathfrak{h}$-submodule generated by $e_{-(\sigma-\delta)} v_{\lambda}$. Since $\left\langle\delta^{\vee}, \lambda\right\rangle=0$, it follows that $R^{\lambda}$ is a simple $\mathfrak{h}$-module with highest weight $2 l-\left\langle\delta^{\vee}, \sigma\right\rangle$, where $l$ is the maximal integer such that $\sigma-l \delta \in \Delta^{+}$. Note that $\mathrm{p}_{\lambda}(v) \in R^{\lambda}$ since otherwise the inequality $\left\langle\delta^{\vee}, \lambda-\sigma\right\rangle<0$ would imply $e_{\delta} \mathrm{p}_{\lambda}(v) \notin R^{\lambda}$, which is not the case. We conclude that $\mathrm{p}_{\lambda}(v)=d e_{-\delta} e_{\delta} \mathrm{p}_{\lambda}(v)$ for some scalar $d \in \mathbb{k}^{\times}$that depends only on $\sigma$ and $\delta$ (and not on $\lambda)$.

It follows from the previous paragraph that

$$
w=c d \sum_{\lambda \in \mathrm{E}_{\varrho}} e_{-\delta} e_{-(\sigma-\delta)} v_{\lambda}=c d \sum_{\lambda \in \mathrm{E}_{\varrho}}\left[e_{-\delta}, e_{-(\sigma-\delta)}\right] v_{\lambda} .
$$

Recall that $w \neq 0$, therefore $\sigma \in \Delta^{+}$and $w=c^{\prime} \sum_{\lambda \in \mathrm{E}_{\varrho}} e_{-\sigma} v_{\lambda}$ for some $c^{\prime} \in \mathbb{k}^{\times}$. Now the vector $v^{\prime}=v-c^{\prime} e_{-\sigma} x_{0}$ satisfies $\mathrm{p}_{\lambda}(v)=0$ for all $\lambda \in \mathrm{E}_{\varrho}$. Since the assumption $w \neq 0$ implies $\sigma \in \Delta^{+}$, the proof is completed.

Lemma 3.25. Under the assumptions of Proposition 3.24, suppose in addition that $\sigma=$ $\alpha \in \Pi$ and $\mathrm{p}_{\lambda}(v)=0$ for all $\lambda \in \mathrm{E}_{\varrho}$. Then

(a) $\left\langle\alpha^{\vee}, \mu\right\rangle>0$ for all $\mu \in \mathrm{E} \backslash \mathrm{E}_{\varrho}$;

(b) there exists $c \in \mathbb{k}^{\times}$such that

$$
v=c \sum_{\mu \in \mathrm{E} \backslash \mathrm{E}_{\varrho}} \frac{\langle\varrho, \mu\rangle}{\left\langle\alpha^{\vee}, \mu\right\rangle} e_{-\alpha} v_{\mu} .
$$

Proof. Consider the expression $v=\sum_{\mu \in \mathrm{E} \backslash \mathrm{E}_{\varrho}} c_{\mu} e_{-\alpha} v_{\mu}$, where $c_{\mu} \in \mathbb{k}$ for all $\mu \in \mathrm{E} \backslash \mathrm{E}_{\varrho}$. Combining Lemma 3.9 with Corollary 3.12(国) yields $e_{\alpha} v \neq 0$. It then follows from Lemmas 3.10 and 3.11 that $e_{\alpha} v=y x_{0}$ for some $y \in \mathfrak{t}$. In particular, for every $\lambda \in \mathrm{E}_{\varrho}$ the condition $\mathrm{p}_{\lambda}(v)=0$ implies $\lambda(y)=0$. Therefore the restriction of $y$ (regarded as an element of $\mathcal{Q} \otimes_{\mathbb{Z}} \mathbb{k}$ ) to $\mathbb{Z} \Gamma \otimes_{\mathbb{Z}} \mathbb{k}$ is proportional to $\varrho$, and so

$$
e_{\alpha} v=c \sum_{\mu \in \mathrm{E} \backslash \mathrm{E}_{\varrho}}\langle\varrho, \mu\rangle v_{\mu}
$$


for some $c \in \mathbb{k}^{\times}$. On the other hand, one has

$$
e_{\alpha} v=\sum_{\mu \in \mathrm{E} \backslash \mathrm{E}_{\varrho}} c_{\mu} e_{\alpha} e_{-\alpha} v_{\mu}=\sum_{\mu \in \mathrm{E} \backslash \mathrm{E}_{\varrho}} c_{\mu}\left\langle\alpha^{\vee}, \mu\right\rangle v_{\mu} .
$$

Comparing (3.9) with (3.10) we obtain the required results.

Proposition 3.26. Suppose that $\sigma \in \Phi(\Gamma)$ and $\varrho \in \mathcal{K}^{1}(\sigma)$. Then the following conditions are equivalent:

(1) $\varrho \in \mathcal{P}$.

(2) $\sigma \in \Pi$.

Proof. Let $v \in V_{\sigma}^{\left(T_{\text {ad }}\right)}$ be such that $[v] \in \mathcal{T S} \backslash\{0\}$. Taking into account Proposition 3.24 , we may assume that $\mathrm{p}_{\lambda}(v)=0$ for all $\lambda \in \mathrm{E}_{\varrho}$.

(11) $\Rightarrow$ (2) Thanks to Lemma 3.9 there exists $\alpha \in \Pi$ such that $e_{\alpha} v \neq 0$. Assume that $\sigma-\alpha \neq 0$. Then Corollary 3.12 implies $\sigma-\alpha \in \Delta^{+}$and $e_{\alpha} v=c e_{-(\sigma-\alpha)} x_{0}$ for some $c \in \mathbb{K}^{\times}$. It follows that $\left\langle(\sigma-\alpha)^{\vee}, \lambda\right\rangle=0$ for all $\lambda \in \mathrm{E}_{\varrho}$ and $\left\langle(\sigma-\alpha)^{\vee}, \mu\right\rangle>0$ for some $\mu \in \mathrm{E} \backslash \mathrm{E}_{\varrho}$. Consequently, $\operatorname{Supp}(\sigma-\alpha) \subset \mathrm{E}_{\varrho}^{\perp}$ and $\operatorname{Supp}(\sigma-\alpha) \not \subset \mathrm{E}^{\perp}$, which contradicts (11). Thus $\sigma=\alpha$.

(2) $\Rightarrow$ (11) Let $\sigma=\alpha \in \Pi$ and assume that $\varrho \notin \mathcal{P}$. By Lemma 3.23 there exists $\delta \in \Pi$ such that $\iota\left(\delta^{\vee}\right)$ is a positive multiple of $\varrho$. Then $\left\langle\delta^{\vee}, \alpha\right\rangle>0$ and hence $\delta=\alpha$. Applying

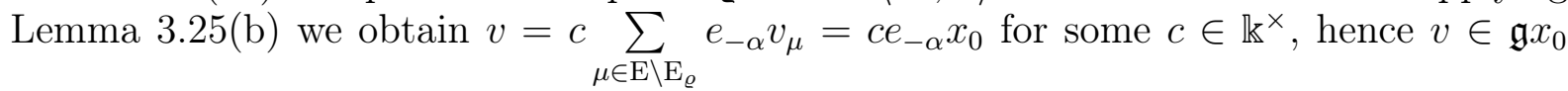
and $[v]=0$, a contradiction.

3.7. Proof of Theorem 3.1; Step 1. The goal of this subsection is to show that every weight $\sigma \in \Phi(\Gamma)$ satisfies conditions $(\Phi 1)-(\Phi 8)$ along with the following one:

(MF) the multiplicity of $\sigma$ in $\mathcal{T S}$ equals 1.

For the rest of this subsection, we fix a weight $\sigma \in \Phi(\Gamma)$ and a vector $v \in V_{\sigma}^{\left(T_{\text {ad }}\right)}$ such that $[v] \in \mathcal{T S} \backslash\{0\}$. Recall the set $\mathcal{K}^{1}(\sigma)$ given by (3.1), which is nonempty by Corollary 3.22 .

Property $(\Phi 1)$ has already been established in Lemma 3.8(b).

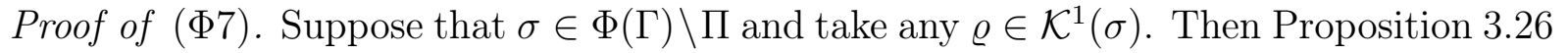
yields $\varrho \notin \mathcal{P}$. By Lemma 3.23 , there exists $\delta \in \Pi$ such that $\iota\left(\delta^{\vee}\right)$ is a positive multiple of $\varrho$. Clearly, $\delta \notin \Gamma^{\perp}$.

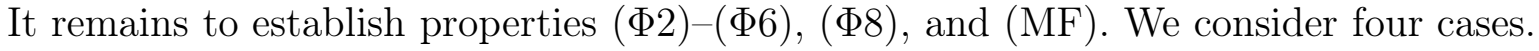

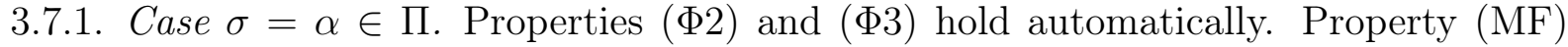
follows from Proposition 3.24 (国) and Lemma $3.25(\mathrm{~b})$. It remains to prove (\$8).

Lemma 3.27. Suppose that $\varrho \in \mathcal{K}^{1}(\alpha)$. Then $\langle\varrho, \alpha\rangle=1$.

Proof. By Proposition 3.24(国) we may assume that $\mathrm{p}_{\lambda}(v)=0$ for all $\lambda \in \mathrm{E}_{\varrho}$. Note that $\varrho \in \mathcal{P}$ by Proposition 3.26. Then it follows from Corollary 3.15 that the section $s \in H^{0}\left(O, \mathcal{N}_{O}\right)^{G}$ given by $s\left(x_{0}\right)=[v]$ extends to $O \cup O_{\varrho}$. Taking into account Lemmas 3.25 and 3.6 along with Proposition 3.18((C), we get $\langle\varrho, \alpha\rangle=1$.

Lemma 3.28. There are inequalities $1 \leq\left|\mathcal{K}^{1}(\alpha)\right| \leq 2$. 
Proof. As $\mathcal{K}^{1}(\alpha)$ is nonempty, we have $\left|\mathcal{K}^{1}(\alpha)\right| \geq 1$. To prove the second inequality, assume that $\varrho_{1}, \varrho_{2}, \varrho_{3} \in \mathcal{K}^{1}(\alpha)$ are three distinct elements. Since $\mathbb{Q}^{+} \varrho_{i}$ is an extremal ray of $\mathcal{K}$ for each $i=1,2,3$, the elements $\varrho_{1}, \varrho_{2}, \varrho_{3}$ are linearly independent in $\mathcal{Q}$. By Proposition 3.24(国), for each $i=1,2,3$ there exist $v_{i} \in V_{\alpha}^{\left(T_{\text {ad }}\right)}$ and $c_{i} \in \mathbb{k}$ such that $v_{i}=v-c_{i} e_{-\alpha} x_{0}$ and $\mathrm{p}_{\lambda}\left(v_{i}\right)=0$ for all $\lambda \in \mathrm{E}_{\varrho_{i}}$. In view of Lemma 3.25(b), for each $i=1,2,3$ there exists $c_{i}^{\prime} \in \mathbb{R}^{\times}$such that $e_{\alpha} v_{i}=c_{i}^{\prime} \sum_{\mu \in \mathrm{E}}\left\langle\varrho_{i}, \mu\right\rangle v_{\mu}$. Obviously, the vectors $e_{\alpha} v_{1}, e_{\alpha} v_{2}$, and $e_{\alpha} v_{3}$ are linearly independent in $V$, hence so are the vectors $v_{1}, v_{2}$, and $v_{3}$. The latter contradicts the fact that $v_{1}, v_{2}, v_{3}$ belong to the linear span of the two vectors $v$ and $e_{-\alpha} x_{0}$.

Proof of (\$8). According to Lemma 3.28, we consider two cases.

Case 1: $\mathcal{K}^{1}(\alpha)$ contains a unique element $\varrho_{0}$. Then $\left\langle\varrho_{0}, \alpha\right\rangle=1$ by Lemma 3.27 and $\langle\varrho, \alpha\rangle \leq 0$ for all $\varrho \in \mathcal{K}^{1} \backslash\left\{\varrho_{0}\right\}$. Put $\varrho_{1}=\varrho_{0}$ and $\varrho_{2}=\iota\left(\alpha^{\vee}\right)-\varrho_{0}$. Proposition 3.26 yields $\varrho_{0} \in \mathcal{P}$, hence $\varrho_{0}$ is not proportional to $\iota\left(\alpha^{\vee}\right)$ and so $\varrho_{1} \neq \varrho_{2}$. Further, $\varrho_{1}, \varrho_{2}$ obviously satisfy conditions $(\Phi 8)(\mathbf{a}, \mathbb{C})$. To complete the proof, it suffices to show that $\varrho_{2} \in \mathcal{K}$. For that, take any $\mu \in \mathrm{E} \backslash \mathrm{E}_{\varrho_{0}}$. Clearly, there is a unique expression $\alpha=\tau+b \mu$ where $\tau \in \mathbb{Q} \mathrm{E}_{\varrho_{0}}$ and $b \in \mathbb{Q}$. Since $\left\langle\varrho_{0}, \alpha\right\rangle=1$, one has $b=1 /\left\langle\varrho_{0}, \mu\right\rangle$. Then $\tau=\alpha-\mu /\left\langle\varrho_{0}, \mu\right\rangle$. One easily checks that $\langle\varrho, \tau\rangle \leq 0$ for all $\varrho \in \mathcal{K}^{1} \backslash\left\{\varrho_{0}\right\}$, hence there is an expression $\tau=-\sum_{\lambda \in \mathrm{E}_{\varrho_{0}}} c_{\lambda} \lambda$ with $c_{\lambda} \in \mathbb{Q}^{+}$for all $\lambda \in \mathrm{E}_{\varrho_{0}}$. Consequently,

$$
\begin{aligned}
&\left\langle\varrho_{2}, \mu\right\rangle=\left\langle\alpha^{\vee}-\varrho_{0}, \mu\right\rangle=\left\langle\varrho_{0}, \mu\right\rangle \cdot\left\langle\alpha^{\vee}-\varrho_{0}, \alpha-\tau\right\rangle= \\
&\left\langle\varrho_{0}, \mu\right\rangle \cdot\left(1+\left\langle\alpha^{\vee}, \sum_{\lambda \in \mathrm{E}_{\varrho_{0}}} c_{\lambda} \lambda\right\rangle\right) \geq\left\langle\varrho_{0}, \mu\right\rangle>0
\end{aligned}
$$

and $\left\langle\varrho_{2}, \lambda\right\rangle=\left\langle\alpha^{\vee}-\varrho_{0}, \lambda\right\rangle=\left\langle\alpha^{\vee}, \lambda\right\rangle \geq 0$ for all $\lambda \in \mathrm{E}_{\varrho_{0}}$. Thus $\varrho_{2} \in \mathcal{K}$.

Case 2: $\mathcal{K}^{1}(\alpha)$ consists of two distinct elements $\varrho_{1}$ and $\varrho_{2}$. We claim that $\varrho_{1}, \varrho_{2}$ satisfy

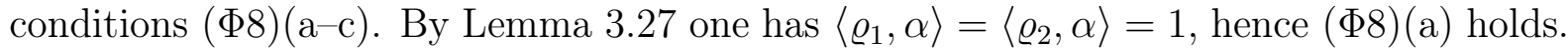
Condition ( $(\Phi 8)($ (C) holds automatically. It remains to prove $(\Phi 8)(\mathrm{B})$.

Lemma 3.29. The cone $\mathbb{Q}^{+} \varrho_{1}+\mathbb{Q}^{+} \varrho_{2} \subset \mathcal{Q}$ is a (two-dimensional) face of the cone $\mathcal{K}$.

Proof. Since $\mathbb{Q}^{+} \varrho_{1}$ is an extremal ray of $\mathcal{K}$, there exists an element $\nu_{1} \in \mathbb{Q}^{+} \Gamma$ such that $\left\langle\varrho_{1}, \nu_{1}\right\rangle=0,\left\langle\varrho_{2}, \nu_{1}\right\rangle=1$, and $\left\langle\varrho, \nu_{1}\right\rangle>0$ for all $\varrho \in \mathcal{K}^{1} \backslash\left\{\varrho_{1}, \varrho_{2}\right\}$. Similarly, there exists an element $\nu_{2} \in \mathbb{Q}^{+} \Gamma$ such that $\left\langle\varrho_{2}, \nu_{2}\right\rangle=0,\left\langle\varrho_{1}, \nu_{2}\right\rangle=1$, and $\left\langle\varrho, \nu_{2}\right\rangle>0$ for all $\varrho \in \mathcal{K}^{1} \backslash\left\{\varrho_{1}, \varrho_{2}\right\}$. Put $\nu=\nu_{1}+\nu_{2}-\alpha$. Then $\left\langle\varrho_{1}, \nu\right\rangle=\left\langle\varrho_{2}, \nu\right\rangle=0$ and $\langle\varrho, \nu\rangle>0$ for all $\varrho \in \mathcal{K}^{1} \backslash\left\{\varrho_{1}, \varrho_{2}\right\}$, hence $\mathbb{Q}^{+} \varrho_{1}+\mathbb{Q}^{+} \varrho_{2}$ is a face of $\mathcal{K}$.

It follows from Lemma 3.29 that the space $\mathbb{Q}\left(\mathrm{E}_{\varrho_{1}} \cap \mathrm{E}_{\varrho_{2}}\right)$ has codimension 2 in $\mathbb{Q} \Gamma$. By Proposition $3.24($ (a) $)$, there exist $v_{1}, v_{2} \in V_{\alpha}^{\left(T_{\text {ad }}\right)}$ such that $\left[v_{1}\right]=\left[v_{2}\right]=[v]$ and

$$
\mathrm{p}_{\lambda}\left(v_{i}\right)=0 \quad \text { for all } \quad \lambda \in \mathrm{E}_{\varrho_{i}}, i=1,2 .
$$

Lemma 3.25 yields $v_{1} \neq v_{2}$, and so $v_{1}-v_{2} \in \mathfrak{g} x_{0} \backslash\{0\}$, which by Lemma 3.11 implies $v_{1}-v_{2}=c e_{-\alpha} x_{0}$ for some $c \in \mathbb{k}^{\times}$. It then follows from (3.11) that $\left\langle\alpha^{\vee}, \lambda\right\rangle=0$ for all $\lambda \in \mathrm{E}_{\varrho_{1}} \cap \mathrm{E}_{\varrho_{2}}$, therefore $\iota\left(\alpha^{\vee}\right)=a_{1} \varrho_{1}+a_{2} \varrho_{2}$ for some $a_{1}, a_{2} \in \mathbb{Q}^{+}$. In view of Lemma 3.25(a) one has $\left\langle\alpha^{\vee}, \mu\right\rangle>0$ for all $\mu \in \mathrm{E} \backslash\left(\mathrm{E}_{\varrho_{1}} \cap \mathrm{E}_{\varrho_{2}}\right)$, whence $a_{i} \neq 0$ for $i=1,2$, which proves (\$8) (b)). 
3.7.2. Case $\sigma \in \Delta^{+} \backslash \Pi$. We need to prove properties $(\Phi 2)-(\Phi 4)$ and $(\underline{\mathrm{MF}})$. In what follows, we fix an arbitrary element $\varrho \in \mathcal{K}^{1}(\sigma)$.

Lemma 3.30. For every $\delta \in \Pi$ there exist $v^{\prime} \in V_{\sigma}^{\left(T_{\mathrm{ad}}\right)}$ and $c \in \mathbb{k}$ such that $v^{\prime}=v-c e_{-\sigma} x_{0}$ and $e_{\delta} v^{\prime}=0$.

Proof. Take any $\delta \in \Pi$ and assume that $e_{\delta} v \neq 0$. Then Corollary 3.12 yields $\sigma-\delta \in \Delta^{+}$ and $e_{\delta} v=c e_{-(\sigma-\delta)} x_{0}$ for some $c \in \mathbb{k}^{\times}$. Then the vector $v^{\prime}=v-c N_{\delta,-\sigma}^{-1} e_{-\sigma} x_{0}$ satisfies $e_{\delta} v^{\prime}=0$.

Lemma 3.31. The set $\left\{\delta \in \operatorname{Supp} \sigma \mid \sigma-\delta \in \Delta^{+}\right\}$contains at least two elements.

Proof. Thanks to Lemma [3.9, there exists $\beta \in \Pi$ such that $e_{\beta} v \neq 0$. Then $\sigma-\beta \in \Delta^{+}$ by Corollary 3.12. Next, by Lemma 3.30 there exists $v^{\prime} \in V_{\sigma}^{\left(T_{\text {ad }}\right)}$ such that $\left[v^{\prime}\right]=[v]$ and $e_{\beta} v^{\prime}=0$. Again, there exists $\gamma \in \Pi$ such that $e_{\gamma} v^{\prime} \neq 0$, which implies $\sigma-\gamma \in \Delta^{+}$. Clearly, $\beta \neq \gamma$ and $\beta, \gamma \in \operatorname{Supp} \sigma$.

Lemma 3.32. One of the following two alternatives holds.

(1) $\left\langle\delta^{\vee}, \sigma\right\rangle \geq 0$ for all $\delta \in \operatorname{Supp} \sigma$ (that is, $\sigma$ is a dominant root of $\Delta_{\sigma}$ ).

(2) $\operatorname{Supp} \sigma$ is of type $\mathrm{G}_{2}$ and $\sigma=\alpha_{1}+\alpha_{2}$.

Proof. In view of Proposition 3.24((a) , we may assume that

$$
\mathrm{p}_{\lambda}(v)=0 \text { for all } \lambda \in \mathrm{E}_{\varrho} .
$$

Thanks to Lemma 3.9, there exists $\alpha \in \Pi$ such that $e_{\alpha} v \neq 0$. Then Corollary 3.12 yields $\sigma-\alpha \in \Delta^{+}$and $e_{\alpha} v=c e_{-(\sigma-\alpha)} x_{0}$ for some $c \in \mathbb{k}^{\times}$. By (3.12) the latter implies $\left\langle(\sigma-\alpha)^{\vee}, \lambda\right\rangle=0$ for all $\lambda \in \mathrm{E}_{\varrho}$. Thus for every $\delta \in \operatorname{Supp}(\sigma-\alpha)$ one has $\delta \in \mathrm{E}_{\varrho}^{\perp}$, whence $\iota\left(\delta^{\vee}\right)$ is a non-negative multiple of $\varrho$. As $\langle\varrho, \sigma\rangle>0$, it follows that $\left\langle\delta^{\vee}, \sigma\right\rangle \geq 0$ for all $\delta \in \operatorname{Supp}(\sigma-\alpha)$. Now assume that $\left\langle\alpha^{\vee}, \sigma\right\rangle<0$. Then $\left\langle\alpha^{\vee}, \sigma\right\rangle \leq-1$ and $\left\langle\alpha^{\vee}, \sigma-\alpha\right\rangle \leq-3$. The latter implies that $\operatorname{Supp} \sigma$ has type $\mathrm{G}_{2}$ with $\alpha=\alpha_{1}$ and $\sigma=\alpha_{1}+\alpha_{2}$.

Proof of (\$2). Applying Lemma 3.31 we find that $\sigma$ cannot be the highest root of $\Delta_{\sigma}$ unless the support of $\sigma$ has type $\mathrm{A}_{r}$. (The latter can be seen, for instance, by inspecting the extended Dynkin diagrams.) By the same reason $\sigma$ cannot be the short dominant root in type $\mathrm{G}_{2}$. All the other possibilities given by Lemma 3.32 are already contained in $\bar{\Sigma}(G)$.

Proof of (\$3). Reasoning as in the proof of Lemma 3.32, we find a simple root $\alpha \in \Pi$ such that $\sigma-\alpha \in \Delta^{+}$and $\iota\left(\delta^{\vee}\right)$ is a non-negative multiple of $\varrho$ for all $\delta \in \operatorname{Supp}(\sigma-\alpha)$. We claim that $\Pi_{\sigma} \subset \operatorname{Supp}(\sigma-\alpha)$. Indeed, the inclusion $\left(\Pi_{\sigma} \backslash\{\alpha\}\right) \subset \operatorname{Supp}(\sigma-\alpha)$ is obvious and the condition $\alpha \in \Pi_{\sigma}$ implies $\alpha \in \operatorname{Supp}(\sigma-\alpha)$ in view of (3.2). Thus, given any $\beta \in \Pi_{\sigma}$, the element $\iota\left(\beta^{\vee}\right)$ is a non-negative multiple of $\varrho$. As $\beta \in \sigma^{\perp}$ and $\langle\varrho, \sigma\rangle>0$, it follows that $\iota\left(\beta^{\vee}\right)=0$, whence $\beta \in \Gamma^{\perp}$.

Proof of (\$4). Suppose that $\sigma=\alpha_{1}+\ldots+\alpha_{r}$ with Supp $\sigma$ of type $\mathrm{B}_{r}(r \geq 2)$. Taking into account Lemma 3.30 we may assume that $e_{\alpha_{r}} v=0$. For $2 \leq i \leq r-1$, we have $\sigma-\alpha_{i} \notin \Delta^{+}$, which implies $e_{\alpha_{i}} v=0$ by Corollary 3.12(国). Now assume that $\alpha_{r} \in \Gamma^{\perp}$.

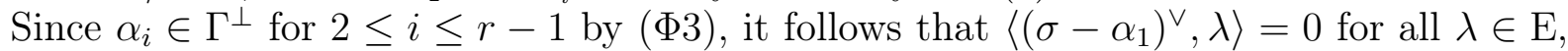
whence $e_{-\left(\sigma-\alpha_{1}\right)} x_{0}=0$. In view of Lemmas 3.10 and 3.11 the latter implies $e_{\alpha_{1}} v=0$. We have obtained that $e_{\delta} v=0$ for all $\delta \in \operatorname{Supp} \sigma$ and hence for all $\delta \in \Pi$, which contradicts Lemma 3.9 as $\sigma \neq 0$. 
Proof of (MF). Here we use a short argument from the proof of [BVS16, Proposition 3.16]. A case-by-case check of all relevant entries in Table 1 shows that the set

$$
\left\{\delta \in \Pi \mid \sigma-\delta \in \Delta^{+}\right\}
$$

contains exactly two elements, which will be denoted by $\beta$ and $\gamma$. Let $v^{\prime} \in V_{\sigma}^{\left(T_{\text {ad }}\right)}$ be another vector such that $\left[v^{\prime}\right] \in \mathcal{T S} \backslash\{0\}$. By Lemma 3.30 we may assume that $e_{\beta} v=$ $e_{\beta} v^{\prime}=0$. It follows from Corollary 3.12 that $e_{\delta} v=e_{\delta} v^{\prime}=0$ for all $\delta \in \Pi \backslash\{\gamma\}$. Consequently, $e_{\gamma} v \neq 0$ and $e_{\gamma} v^{\prime} \neq 0$ in view of Lemma 3.9. Then Corollary 3.12(b) yields $e_{\gamma} v=c e_{-(\sigma-\gamma)} x_{0}$ and $e_{\gamma} v^{\prime}=c^{\prime} e_{-(\sigma-\gamma)} x_{0}$ for some $c, c^{\prime} \in \mathbb{k}^{\times}$. It follows that the vector $c^{\prime} v-c v^{\prime}$ is annihilated by $e_{\gamma}$ and hence by all $e_{\delta}$ with $\delta \in \Pi$. As $\sigma \neq 0$, Lemma 3.9 yields $c^{\prime} v-c v^{\prime}=0$.

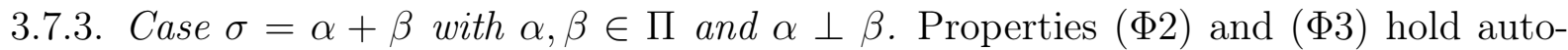
matically. Properties (\$5) and (MF) follow from the lemma below.

Lemma 3.33. The following assertions hold:

(a) $\iota\left(\alpha^{\vee}\right)=\iota\left(\beta^{\vee}\right)$;

(b) the vector $v$ is given by

$$
v=c \sum_{\mu \in \mathrm{E}:\left\langle\alpha^{\vee}, \mu\right\rangle>0} \frac{1}{\left\langle\alpha^{\vee}, \mu\right\rangle} e_{-\alpha} e_{-\beta} v_{\mu}
$$

for some $c \in \mathbb{k}^{\times}$.

Proof. Take any $\varrho \in \mathcal{K}^{1}(\sigma)$. Proposition 3.26 yields $\varrho \notin \mathcal{P}$. Then by Lemma 3.23 there exists $\delta \in \Pi$ such that $\iota\left(\delta^{\vee}\right)$ is a positive multiple of $\varrho$. As $\left\langle\delta^{\vee}, \sigma\right\rangle>0$, it follows that $\delta \in\{\alpha, \beta\}$. Assume without loss of generality that $\delta=\alpha$. Proposition 3.24(b) yields $\mathrm{p}_{\lambda}(v)=0$ for all $\lambda \in \mathrm{E}_{\varrho}$. Next, for every $\mu \in \mathrm{E} \backslash \mathrm{E}_{\varrho}$ one has

$$
\mathrm{p}_{\mu}(v)=c_{\mu} e_{-\alpha} e_{-\beta} v_{\mu}=c_{\mu} e_{-\beta} e_{-\alpha} v_{\mu}
$$

with $c_{\mu} \in \mathbb{k}$. It follows that

$$
e_{\alpha} v=\sum_{\mu \in \mathrm{E} \backslash \mathrm{E}_{\varrho}} c_{\mu} e_{\alpha} e_{-\alpha} e_{-\beta} v_{\mu}=\sum_{\mu \in \mathrm{E} \backslash \mathrm{E}_{\varrho}} c_{\mu}\left\langle\alpha^{\vee}, \mu\right\rangle e_{-\beta} v_{\mu} .
$$

On the other hand, since $v \neq 0$, there exists $\nu \in \mathrm{E} \backslash \mathrm{E}_{\varrho}$ such that $\mathrm{p}_{\nu}(v) \neq 0$, which implies $c_{\nu} \neq 0$ and $e_{-\beta} v_{\nu} \neq 0$ in view of (3.13). It follows that $e_{\alpha} v \neq 0$, and Corollary $3.12(\mathrm{~b})$ yields

$$
e_{\alpha} v=c e_{-\beta} x_{0}=c \sum_{\lambda \in \mathrm{E}} e_{-\beta} v_{\lambda}
$$

for some $c \in \mathbb{k}^{\times}$. Then $\left\langle\beta^{\vee}, \lambda\right\rangle=0$ for all $\lambda \in \mathrm{E}_{\varrho}$, whence $\iota\left(\beta^{\vee}\right)$ and $\iota\left(\alpha^{\vee}\right)$ are proportional. The equalities $\left\langle\alpha^{\vee}, \sigma\right\rangle=2=\left\langle\beta^{\vee}, \sigma\right\rangle$ imply $\iota\left(\alpha^{\vee}\right)=\iota\left(\beta^{\vee}\right)$, which proves (国). Now comparing (3.14) with (3.15) yields (b). 
3.7.4. Case $\sigma \notin \Delta^{+}$and $\sigma$ is not the sum of two orthogonal simple roots. We need to prove properties $(\underline{\Phi 2}),(\underline{\Phi 3}),(\underline{\Phi 6})$, and $(\underline{\mathrm{MF}})$. In what follows, we fix an arbitrary element $\varrho \in \mathcal{K}^{1}(\sigma)$.

The following lemma is similar to [BVS16, Proposition 3.9].

Lemma 3.34. There exists a unique $\beta \in \Pi$ such that $\sigma-\beta \in \Delta^{+}$.

Proof. By Lemma 3.9 there exists $\beta \in \Pi$ such that $e_{\beta} v \neq 0$. Then Corollary 3.12(勾) yields $\sigma-\beta \in \Delta^{+}$. The condition $\sigma \notin \Delta^{+}$implies $\left\langle\beta^{\vee}, \sigma-\beta\right\rangle \geq 0$, and so $\left\langle\beta^{\vee}, \sigma\right\rangle \geq 2$. Now take any $\gamma \in \Pi \backslash\{\beta\}$ and assume that $\sigma-\gamma \in \Delta^{+}$. Clearly,

$$
\left\langle\beta^{\vee}, \sigma-\gamma\right\rangle=\left\langle\beta^{\vee}, \sigma\right\rangle-\left\langle\beta^{\vee}, \gamma\right\rangle \geq 2>0,
$$

whence $\sigma-\beta-\gamma \in \Delta^{+}$(note that $\sigma \neq \beta+\gamma$ by our assumptions). Let $\mathfrak{h} \subset \mathfrak{g}$ be the standard Levi subalgebra with set of simple roots $\{\beta, \gamma\}$ and regard $\mathfrak{g}$ as an $\mathfrak{h}$-module. As $\left\langle\beta^{\vee}, \sigma-\gamma+\beta\right\rangle \geq 4$, one has $\sigma-\gamma+\beta \notin \Delta^{+}$. Consequently, $e_{\sigma-\gamma}$ is a highest weight vector for $\mathfrak{h}$. Similarly, $e_{\sigma-\beta}$ is another highest weight vector for $\mathfrak{h}$. Since $e_{\sigma-\beta-\gamma}$ is proportional to both $\left[e_{-\beta}, e_{\sigma-\gamma}\right]$ and $\left[e_{-\gamma}, e_{\sigma-\beta}\right]$, it follows that $e_{\sigma-\beta-\gamma}$ is contained in two different simple $\mathfrak{h}$-submodules of $\mathfrak{g}$, a contradiction.

Until the end of this case, $\beta$ stands for the unique simple root such that $\sigma-\beta \in \Delta^{+}$.

Lemma 3.35. The following assertions hold:

(a) $e_{\beta} v \neq 0$;

(b) $e_{\beta} v=c e_{-(\sigma-\beta)} x_{0}$ for some $c \in \mathbb{k}^{\times}$;

(c) $\iota\left((\sigma-\beta)^{\vee}\right)$ is a positive multiple of $\varrho$.

Proof. By Lemma 3.9 there exists $\delta \in \Pi$ such that $e_{\delta} v \neq 0$. Corollary 3.12(国) and Lemma 3.34 then yield $\delta=\beta$, whence part (国). Part (b) is implied by Corollary 3.12(b) . For every $\lambda \in \mathrm{E}_{\varrho}$, one has $\mathrm{p}_{\lambda}(v)=0$ by Proposition $3.24(\mathrm{~b})$, hence $\left\langle(\sigma-\beta)^{\vee}, \lambda\right\rangle=0$. Thus $\iota\left((\sigma-\beta)^{\vee}\right)$ is a non-negative multiple of $\varrho$. As $e_{\beta} v \neq 0$, it follows that $\iota\left((\sigma-\beta)^{\vee}\right) \neq 0$, whence part (ㄷ) .

Lemma 3.36. Suppose that $\gamma \in \Pi \backslash\{\beta\}$ is such that $\sigma-\beta-\gamma \in \Delta^{+}$. Then $\left\langle\beta^{\vee}, \gamma\right\rangle<0$ (that is, $\beta+\gamma \in \Delta^{+}$).

Proof. By Lemma 3.35(b) one has $e_{\beta} v=c e_{-(\sigma-\beta)} x_{0}$ for some $c \in \mathbb{k}^{\times}$. Assume that $\beta$ and $\gamma$ are orthogonal. Then $\beta+\gamma \notin \Delta^{+}$, and so $e_{\sigma-\beta-\gamma} v=0$ by Corollary 3.12(包). As $\beta \neq \gamma$, it follows from Lemma 3.34 and Corollary 3.12 (国) that $e_{\gamma} v=0$. Then $e_{\sigma-\beta} v=$ $N_{\gamma, \sigma-\beta-\gamma}^{-1} e_{\gamma} e_{\sigma-\beta-\gamma} v=0$. Hence in view of Lemma 3.35(ㄷ) one has

$$
0=e_{\beta} e_{\sigma-\beta} v=e_{\sigma-\beta} e_{\beta} v=e_{\sigma-\beta}\left(c e_{-(\sigma-\beta)} x_{0}\right)=c h_{\sigma-\beta} x_{0} \neq 0,
$$

a contradiction.

Lemma 3.37. For every $\delta \in \operatorname{Supp} \sigma$, the element $\iota\left(\delta^{\vee}\right)$ is a non-negative multiple of $\varrho$.

Proof. By Lemma 3.35(ㄷ), the element $\iota\left((\sigma-\beta)^{\vee}\right)$ is a positive multiple of $\varrho$. Since $(\sigma-\beta)^{\vee}=\sum_{\delta \in \operatorname{Supp}(\sigma-\beta)} c_{\delta} \delta^{\vee}$ with all the coefficients $c_{\delta}$ being positive, it follows that $\left\langle\delta^{\vee}, \lambda\right\rangle=0$ for all $\delta \in \operatorname{Supp}(\sigma-\beta)$ and $\lambda \in \mathrm{E}_{\varrho}$. Consequently, $\iota\left(\delta^{\vee}\right)$ is a non-negative multiple of $\varrho$ for all $\delta \in \operatorname{Supp}(\sigma-\beta)$. It remains to show that $\operatorname{Supp}(\sigma-\beta)=\operatorname{Supp} \sigma$ or, equivalently, $\beta \in \operatorname{Supp}(\sigma-\beta)$. Assume the converse and choose $\gamma \in \operatorname{Supp}(\sigma-\beta)$ such 
that $\sigma-\beta-\gamma \in \Delta^{+}$. (The latter is possible because $\sigma-\beta \notin \Pi$.) Then $\left\langle\beta^{\vee}, \gamma\right\rangle<0$ by Lemma 3.36, hence $\left\langle\beta^{\vee}, \sigma-\beta\right\rangle<0$. The latter yields $\sigma=\beta+(\sigma-\beta) \in \Delta^{+}$, a contradiction.

Proof of $(\Phi 2)$. The key idea of our proof is to reduce the consideration to the case where $V$ is a simple $G$-module, which has already been investigated in Ja07.

Replacing $G$ with a suitable finite cover, we may assume that $G=G_{0} \times C$ where $G_{0}$ is a simply connected semisimple group and $C$ is a torus. Let $L$ denote the standard Levi subgroup of $G$ with set of simple roots $\operatorname{Supp} \sigma$ and let $L^{\prime}$ be the derived subgroup of $L$. Put $T^{\prime}=L^{\prime} \cap T$, so that $T^{\prime}$ is a maximal torus of $L^{\prime}$, and consider the natural restriction $\operatorname{map} \pi: \mathfrak{X}(T) \rightarrow \mathfrak{X}\left(T^{\prime}\right)$.

Lemma 3.35(b) says that $e_{\beta} v=c e_{-(\sigma-\beta)} x_{0}$ for some $c \in \mathbb{k}^{\times}$. By Lemma 3.6 there exists $\nu \in \mathrm{E} \backslash \mathrm{E}_{\varrho}$ such that $\langle\varrho, \nu\rangle=1$. It then follows from Lemma 3.35)(ㄷ) that $\left\langle(\sigma-\beta)^{\vee}, \nu\right\rangle>0$, whence $e_{-(\sigma-\beta)} v_{\nu} \neq 0$. Consequently, $e_{\beta} \mathrm{p}_{\nu}(v) \neq 0$ and $\mathrm{p}_{\nu}(v) \neq 0$.

Let $W \subset V(\nu)$ be the $L$-submodule generated by $v_{\nu}$. Note that $W \cap \mathfrak{g} v_{\nu}=\mathfrak{l}^{\prime} v_{\nu}$. Since $\sigma \notin \Delta^{+} \cup\{0\}$, one has $\mathrm{p}_{\nu}(v) \notin \mathfrak{l}^{\prime} v_{\nu}$, therefore the image of $\mathrm{p}_{\nu}(v)$ in $W / \mathfrak{l}^{\prime} v_{\nu}$ is nonzero. We now show that this image is $L_{v_{\nu}}^{\prime}$-invariant.

First of all, we prove that

$$
e_{\delta} \mathrm{p}_{\nu}(v) \in \mathfrak{l}^{\prime} v_{\nu} \text { for all } \delta \in \Delta_{\sigma} \text { with } e_{\delta} \in \mathfrak{l}_{v_{\nu}}^{\prime} .
$$

Take any such $\delta$. It suffices to show that

$$
e_{\delta} \in \mathfrak{g}_{x_{0}},
$$

because the latter implies $e_{\delta} v \in \mathfrak{g} x_{0}$ and $e_{\delta} \mathrm{p}_{\nu}(v) \in \mathfrak{g} v_{\nu} \cap W=\mathfrak{l}^{\prime} v_{\nu}$. If $\delta \in \Delta^{+}$then (3.17) holds automatically. Now assume that $\delta \in \Delta^{-}$. Then $\operatorname{Supp} \delta \subset \operatorname{Supp} \sigma$, hence $\iota\left(\delta^{\vee}\right)$ is a multiple of $\varrho$ by Lemma 3.37. Since $e_{\delta} v_{\nu}=0$, it follows that $\left\langle\delta^{\vee}, \nu\right\rangle=0$. The latter implies $\iota\left(\delta^{\vee}\right)=0$, whence (3.17).

Next, we prove that

$$
t \cdot \mathrm{p}_{\nu}(v)=\mathrm{p}_{\nu}(v) \text { for all } t \in T_{v_{\nu}}^{\prime} .
$$

The latter claim will follow as soon as we prove that $T_{v_{\nu}}^{\prime} \subset T_{x_{0}}$. Since

$$
T_{v_{\nu}}^{\prime}=\left\{t \in T \mid t^{\lambda}=1 \text { for all } \lambda \in \operatorname{Ker} \pi+\mathbb{Z} \nu\right\},
$$

it suffices to show that $\mathrm{E} \subset \operatorname{Ker} \pi+\mathbb{Z} \nu$. Observe that the lattice $\operatorname{Ker} \pi$ is generated by all elements of $\mathfrak{X}(C)$ and all fundamental weights of $G_{0}$ corresponding to simple roots in the set $\Pi \backslash \operatorname{Supp} \sigma$. Since $\delta \in \mathrm{E}_{\varrho}^{\perp}$ for all $\delta \in \operatorname{Supp} \sigma$ (see Lemma 3.37), we have $\mathrm{E}_{\varrho} \subset \operatorname{Ker} \pi \subset \operatorname{Ker} \pi+\mathbb{Z} \nu$. Next, for every $\mu \in \mathrm{E} \backslash \mathrm{E}_{\varrho}$, there is a unique expression $\mu=\tau+b \nu$ with $\tau \in \mathbb{Q E}_{\varrho}$ and $b \in \mathbb{Q}$. Since $\langle\varrho, \nu\rangle=1$, we have $b=\langle\varrho, \mu\rangle \in \mathbb{Z}$ and $\tau=\mu-\langle\varrho, \mu\rangle \nu \in \mathbb{Z} \Gamma \cap \mathbb{Q E}_{\varrho}=\mathbb{Z} \mathrm{E}_{\varrho}$, which implies $\mu \in \operatorname{Ker} \pi+\mathbb{Z} \nu$.

It follows from (3.16) and (3.18) that the image of $\mathrm{p}_{\nu}(v)$ in $W / \mathfrak{l}^{\prime} v_{\nu}$ is a nonzero element of $\left(W / \mathfrak{l}^{\prime} v_{\nu}\right)^{L_{v_{\nu}}^{\prime}}$ (compare with Lemma 3.7). Now results of [Ja07, § 1.3] (see Proposition 1.6 in loc. cit. and its proof) imply that $\sigma \in \bar{\Sigma}(G)$.

Proof of (\$3). Let $\delta \in \Pi_{\sigma}$. Then $\left\langle\delta^{\vee}, \sigma\right\rangle=0$ by (3.2). Lemma 3.37 implies that $\iota\left(\delta^{\vee}\right)$ is a non-negative multiple of $\varrho$. As $\langle\varrho, \sigma\rangle>0$, one has $\iota\left(\delta^{\vee}\right)=0$ and so $\delta \in \Gamma^{\perp}$.

Proof of (\$6). Suppose that $\sigma=2 \alpha$ with $\alpha \in \Pi$. Proposition 3.26 yields $\varrho \notin \mathcal{P}$. Hence by Lemma 3.23 there exist $\delta \in \Pi$ and a positive integer $n$ such that $\iota\left(\delta^{\vee}\right)=n \varrho$. In particular, 
we obtain $\left\langle\delta^{\vee}, \sigma\right\rangle>0$, whence $\delta=\alpha$. Note that for every $\mu \in \mathrm{E} \backslash \mathrm{E}_{\varrho}$ one has $\mathrm{p}_{\mu}(v)=$ $c_{\mu} e_{-\alpha} e_{-\alpha} v_{\mu}$ with $c_{\mu} \in \mathbb{k}$. Applying Lemma 3.9 we obtain $e_{\alpha} v \neq 0$. Then Corollary $3.12(\mathrm{~b})$ yields $e_{\alpha} v=c e_{-\alpha} x_{0}$ for some $c \in \mathbb{k}^{\times}$. Consequently, for every $\mu \in \mathrm{E} \backslash \mathrm{E}_{\varrho}$ one has $e_{\alpha} \mathrm{p}_{\mu}(v) \neq 0$ and hence $\mathrm{p}_{\mu}(v) \neq 0$, which implies $e_{-\alpha} e_{-\alpha} v_{\mu} \neq 0$ and therefore $\left\langle\alpha^{\vee}, \mu\right\rangle \geq 2$. In view of Lemma 3.6, the latter yields $n \geq 2$. Since $4 / n=\left\langle\alpha^{\vee} / n, 2 \alpha\right\rangle=\langle\varrho, \sigma\rangle \in \mathbb{Z}$, it follows that $n \in\{2,4\}$. In both cases we obtain $\left\langle\alpha^{\vee}, \mathbb{Z} \Gamma\right\rangle \subset 2 \mathbb{Z}$ as required.

Proof of $(\mathrm{MF})$. Here we again use a short argument from the proof of [BVS16, Proposition 3.16]. Let $v^{\prime} \in V_{\sigma}^{\left(T_{\text {ad }}\right)}$ be another vector such that $\left[v^{\prime}\right] \in \mathcal{T} \mathcal{S} \backslash\{0\}$. By Corollary 3.12(a) and Lemma 3.34 one has $e_{\delta} v=e_{\delta} v^{\prime}=0$ for all $\delta \in \Pi \backslash\{\beta\}$. Next, by Lemma 3.35(b) we have $e_{\beta} v=c e_{-(\sigma-\beta)} x_{0}$ and $e_{\beta} v^{\prime}=c^{\prime} e_{-(\sigma-\beta)} x_{0}$ for some $c, c^{\prime} \in \mathbb{k}^{\times}$. Then the vector $c^{\prime} v-c v^{\prime}$ is annihilated by $e_{\beta}$ and hence by all $e_{\delta}$ with $\delta \in \Pi$. As $\sigma \neq 0$, Lemma 3.9 yields $c^{\prime} v-c v^{\prime}=0$.

3.8. Proof of Theorem 3.1: Step 2. Our goal in this subsection is to prove the following

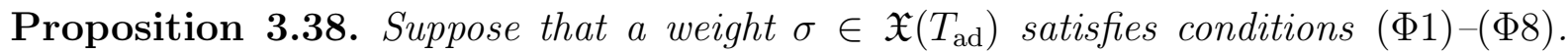
Then $\sigma \in \Phi(\Gamma)$.

In the proof of this proposition we shall need the following lemma.

Lemma 3.39. Suppose that $\sigma \in \mathbb{Z} \Gamma, v \in V_{\sigma}^{\left(T_{\mathrm{ad}}\right)}, e_{\delta} v=0$ for all $\delta \in \Gamma^{\perp}$, and $e_{\delta} v \in \mathfrak{g} x_{0}$ for all $\delta \in \Pi \backslash \Gamma^{\perp}$. Then $[v] \in\left(V / \mathfrak{g} x_{0}\right)^{G_{x_{0}}}$.

Proof. The claim will follow as soon as we check conditions (1) and (2') of Lemma 3.7 . As $\sigma \in \mathbb{Z} \Gamma$, the vector $v$ is $T_{x_{0}}$-invariant, hence so is $[v]$. In view of the hypothesis it now suffices to prove that $e_{-\delta} v=0$ for all $\delta \in \Gamma^{\perp}=\mathrm{E}^{\perp}$. But the latter holds because $e_{\delta} v=0$ and $\left\langle\delta^{\vee}, \lambda-\sigma\right\rangle=0$ for all $\delta \in \mathrm{E}^{\perp}$ and $\lambda \in \mathrm{E}$.

Proof of Proposition 3.38 for $\sigma \in \Pi$. Suppose that $\sigma=\alpha \in \Pi$. Then by ( $\Phi 8)$ there exist

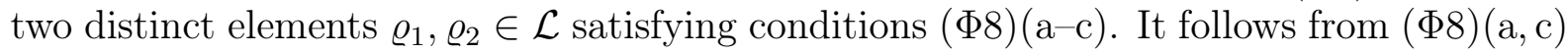
that $\varnothing \neq \mathcal{K}^{1}(\alpha) \subset\left\{\varrho_{1}, \varrho_{2}\right\}$. Further we consider two cases.

Case 1: $\left|\mathcal{K}^{1}(\alpha)\right|=1$. Assume without loss of generality that $\mathcal{K}^{1}(\alpha)=\left\{\varrho_{1}\right\}$. Then condition (\$8) (b) implies $\left\langle\alpha^{\vee}, \mu\right\rangle>0$ for all $\mu \in \mathrm{E} \backslash \mathrm{E}_{\varrho_{1}}$, and we put

$$
v=\sum_{\mu \in \mathrm{E} \backslash \mathrm{E}_{\varrho_{1}}} \frac{\left\langle\varrho_{1}, \mu\right\rangle}{\left\langle\alpha^{\vee}, \mu\right\rangle} e_{-\alpha} v_{\mu}
$$

Clearly, $v \in V_{\alpha}^{\left(T_{\text {ad }}\right)}$ and $e_{\beta} v=0$ for all $\beta \in \Pi \backslash\{\alpha\}$. As $e_{\alpha} v=\sum_{\mu \in \mathrm{E} \backslash \mathrm{E}_{\varrho_{1}}}\left\langle\varrho_{1}, \mu\right\rangle v_{\mu} \in \mathfrak{t} x_{0} \subset \mathfrak{g} x_{0}$, we obtain $[v] \in\left(V / \mathfrak{g} x_{0}\right)^{G_{x_{0}}}$ by Lemma 3.39, Since $\iota\left(\alpha^{\vee}\right)$ is not proportional to $\varrho_{1}$, one has $[v] \neq 0$ (see Lemma 3.11). Applying Proposition 3.18(b, d , and Corollary 3.15 we find that $[v] \in \mathcal{T} \mathcal{S}$, whence $\alpha \in \Phi(\Gamma)$.

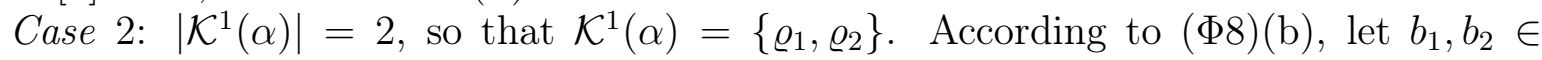
$\mathbb{Q}^{+} \backslash\{0\}$ be such that $\iota\left(\alpha^{\vee}\right)=b_{1} \varrho_{1}+b_{2} \varrho_{2}$. Put

$$
v_{1}=b_{1} \sum_{\mu \in \mathrm{E} \backslash \mathrm{E}_{\varrho_{1}}} \frac{\left\langle\varrho_{1}, \mu\right\rangle}{\left\langle\alpha^{\vee}, \mu\right\rangle} e_{-\alpha} v_{\mu} \text { and } v_{2}=-b_{2} \sum_{\mu \in \mathrm{E} \backslash \mathrm{E}_{\varrho_{2}}} \frac{\left\langle\varrho_{2}, \mu\right\rangle}{\left\langle\alpha^{\vee}, \mu\right\rangle} e_{-\alpha} v_{\mu} \text {. }
$$


Clearly, $v_{1}, v_{2} \in V_{\alpha}^{\left(T_{\text {ad }}\right)}$. As in Case 1 , we see that $\left[v_{1}\right],\left[v_{2}\right] \in\left(V / \mathfrak{g} x_{0}\right)^{G_{x_{0}}} \backslash\{0\}$. An easy check shows that $\left[v_{1}\right]=\left[v_{2}\right]$. Applying Proposition $[3.18(\mathrm{~b}, \mathrm{~d})$ and Corollary 3.15 we find that $\left[v_{1}\right]=\left[v_{2}\right] \in \mathcal{T} \mathcal{S}$ and so $\alpha \in \Phi(\Gamma)$.

Proof of Proposition 3.38 for $\sigma \notin \Pi$. Let $\sigma \in \mathfrak{X}\left(T_{\mathrm{ad}}\right) \backslash \Pi$ and suppose that $\sigma$ satisfies

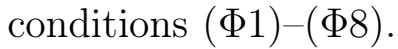

Lemma 3.40. Suppose that $v \in V_{\sigma}^{\left(T_{\mathrm{ad}}\right)}$ and $[v] \in\left(V / \mathfrak{g} x_{0}\right)^{G_{x_{0}}}$. Then $[v] \in \mathcal{T} \mathcal{S}$.

Proof. Let $s \in H^{0}\left(O, \mathcal{N}_{O}\right)^{G}$ be the section defined by $s\left(x_{0}\right)=[v]$. Recall from Corollary 3.15 that $[v] \in \mathcal{T} \mathcal{S}$ if and only if $s$ extends to $O \cup O_{\varrho}$ for each $\varrho \in \mathcal{P}$. We now fix any $\varrho \in \mathcal{P}$ and show that $s$ extends to $O \cup O_{\varrho}$. Assume that $\langle\varrho, \sigma\rangle>0$. Then by (\$7) there exists $\delta \in \Pi$ such that $\iota\left(\delta^{\vee}\right)$ is a positive multiple of $\varrho$. It follows that $\delta \in \mathrm{E}_{\varrho}^{\perp} \backslash \mathrm{E}^{\perp}$, which contradicts the condition $\mathrm{E}^{\perp}=\mathrm{E}_{\varrho}^{\perp}$. Consequently, $\langle\varrho, \sigma\rangle \leq 0$, which implies $[v] \in \mathcal{T} \mathcal{S}$ by Proposition $3.18(\mathrm{~b})$.

To complete the proof, by Lemmas 3.40 and 3.39 it suffices to find a vector $v \in V_{\sigma}^{\left(T_{\text {ad }}\right)}$ with the following properties:

(V1) $v \notin \mathfrak{g} x_{0}$

(V2) $e_{\delta} v=0$ for all $\delta \in \Gamma^{\perp}$;

(V3) $e_{\delta} v \in \mathfrak{g} x_{0}$ for all $\delta \in \Pi \backslash \Gamma^{\perp}$.

In view of condition ( $(\Phi 2)$, it is enough to present such a vector $v$ for each of the cases in Table 1. This is done in the remaining part of the proof. In each case, the explicit formula for $v$ depends on the signs of the structure constants $N_{\alpha, \beta}$ of the Lie algebra [l, $\left.\mathfrak{l}\right]$, where $\mathfrak{l} \subset \mathfrak{g}$ is the standard Levi subalgebra with set of simple roots $\operatorname{Supp} \sigma$; we use the choice of these signs specified in Appendix A.

Case 1: $\sigma \in \Delta^{+}$. By Lemma 3.11 one has $V_{\sigma}^{\left(T_{\text {ad }}\right)} \cap \mathfrak{g} x_{0}=\mathbb{k} e_{-\sigma} x_{0}$.

Subcase 1.1: $\sigma=\alpha_{1}+\ldots+\alpha_{r}$ with $\operatorname{Supp} \sigma$ of type $\mathrm{A}_{r}(r \geq 2)$. Then $\operatorname{Supp} \sigma \cap \sigma^{\perp}=\Pi_{\sigma}$.

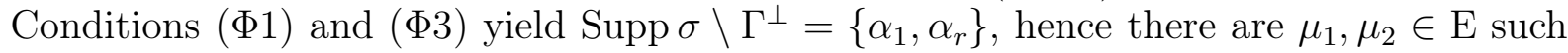
that $e_{-\left(\sigma-\alpha_{r}\right)} v_{\mu_{1}} \neq 0$ and $e_{-\left(\sigma-\alpha_{1}\right)} v_{\mu_{2}} \neq 0$. Consider the element

$$
f=e_{-\left(\alpha_{2}+\ldots+\alpha_{r}\right)} e_{-\alpha_{1}}+e_{-\left(\alpha_{3}+\ldots+\alpha_{r}\right)} e_{-\left(\alpha_{1}+\alpha_{2}\right)}+\ldots+e_{-\alpha_{r}} e_{-\left(\alpha_{1}+\ldots+\alpha_{r-1}\right)} \in U(\mathfrak{g}) .
$$

Direct computations taking into account (\$3) show that

$$
\begin{aligned}
e_{\alpha_{1}} f v_{\lambda} & =\left\langle\alpha_{1}^{\vee}, \lambda\right\rangle e_{-\left(\sigma-\alpha_{1}\right)} v_{\lambda}, & e_{\alpha_{1}} e_{-\sigma} v_{\lambda} & =-e_{-\left(\sigma-\alpha_{1}\right)} v_{\lambda}, \\
e_{\alpha_{r}} f v_{\lambda} & =\left(\left\langle\alpha_{r}^{\vee}, \lambda\right\rangle+r-1\right) e_{-\left(\sigma-\alpha_{r}\right)} v_{\lambda}, & e_{\alpha_{r}} e_{-\sigma} v_{\lambda} & =e_{-\left(\sigma-\alpha_{r}\right)} v_{\lambda} ; \\
e_{\delta} f v_{\lambda} & =0, & e_{\delta} e_{-\sigma} v_{\lambda} & =0
\end{aligned}
$$

for every $\lambda \in \mathrm{E}$ and $\delta \in \operatorname{Supp} \sigma \backslash\left\{\alpha_{1}, \alpha_{r}\right\}$. We now put

$$
v=\sum_{\lambda \in \mathrm{E}} \frac{f+\left\langle\alpha_{1}^{\vee}, \lambda\right\rangle e_{-\sigma}}{\left\langle\alpha_{1}^{\vee}, \lambda\right\rangle+\left\langle\alpha_{r}^{\vee}, \lambda\right\rangle+r-1} v_{\lambda} .
$$

Then $e_{\alpha_{1}} v=0, e_{\alpha_{r}} v=e_{-\left(\sigma-\alpha_{r}\right)} x_{0}$, and $e_{\delta} v=0$ for all $\delta \in \operatorname{Supp} \sigma \backslash\left\{\alpha_{1}, \alpha_{r}\right\}$. Clearly, $e_{\delta} v=0$ for all $\delta \in \Pi \backslash \operatorname{Supp} \sigma$, and we have proved (V2) and (V3). Since $\mathrm{p}_{\mu_{1}}\left(e_{\alpha_{r}} v\right)=$ $e_{-\left(\sigma-\alpha_{r}\right)} v_{\mu_{1}} \neq 0$, we have $v \neq 0$. As $\mathrm{p}_{\mu_{2}}\left(e_{\alpha_{1}} e_{-\sigma} x_{0}\right)=-e_{-\left(\sigma-\alpha_{1}\right)} v_{\mu_{2}} \neq 0$, the vector $v$ is not proportional to $e_{-\sigma} x_{0}$, hence (V1). 
Subcase 1.2: $\sigma=\alpha_{1}+\ldots+\alpha_{r}$ with $\operatorname{Supp} \sigma$ of type $\mathrm{B}_{r}(r \geq 2)$. Then $\operatorname{Supp} \sigma \cap \sigma^{\perp}=$

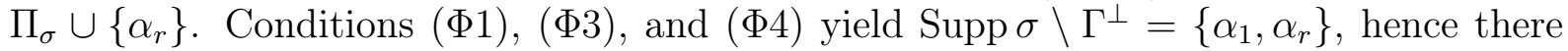
are $\mu_{1}, \mu_{2} \in \mathrm{E}$ such that $e_{-\left(\sigma-\alpha_{r}\right)} v_{\mu_{1}} \neq 0$ and $e_{-\left(\sigma-\alpha_{1}\right)} v_{\mu_{2}} \neq 0$. Consider the element

$$
f=e_{-\left(\alpha_{2}+\ldots+\alpha_{r}\right)} e_{-\alpha_{1}}+e_{-\left(\alpha_{3}+\ldots+\alpha_{r}\right)} e_{-\left(\alpha_{1}+\alpha_{2}\right)}+\ldots+e_{-\alpha_{r}} e_{-\left(\alpha_{1}+\ldots+\alpha_{r-1}\right)} \in U(\mathfrak{g}) .
$$

Direct computations taking into account (\$3) show that

$$
\begin{aligned}
& e_{\alpha_{1}} f v_{\lambda}=\left\langle\alpha_{1}^{\vee}, \lambda\right\rangle e_{-\left(\sigma-\alpha_{1}\right)} v_{\lambda}, \quad e_{\alpha_{1}} e_{-\sigma} v_{\lambda}=-e_{-\left(\sigma-\alpha_{1}\right)} v_{\lambda} \text {, } \\
& e_{\alpha_{r}} f v_{\lambda}=\left(\left\langle\alpha_{r}^{\vee}, \lambda\right\rangle+2 r-2\right) e_{-\left(\sigma-\alpha_{r}\right)} v_{\lambda}, \quad e_{\alpha_{r}} e_{-\sigma} v_{\lambda}=2 e_{-\left(\sigma-\alpha_{r}\right)} v_{\lambda} \text {, } \\
& e_{\delta} f v_{\lambda}=0, \quad e_{\delta} e_{-\sigma} v_{\lambda}=0
\end{aligned}
$$

for every $\lambda \in \mathrm{E}$ and $\delta \in \operatorname{Supp} \sigma \backslash\left\{\alpha_{1}, \alpha_{r}\right\}$. We now put

$$
v=\sum_{\lambda \in \mathrm{E}} \frac{f+\left\langle\alpha_{1}^{\vee}, \lambda\right\rangle e_{-\sigma}}{2\left\langle\alpha_{1}^{\vee}, \lambda\right\rangle+\left\langle\alpha_{r}^{\vee}, \lambda\right\rangle+2 r-2} v_{\lambda} .
$$

Then $e_{\alpha_{1}} v=0, e_{\alpha_{r}} v=e_{-\left(\sigma-\alpha_{r}\right)} x_{0}$, and $e_{\delta} v=0$ for all $\delta \in \operatorname{Supp} \sigma \backslash\left\{\alpha_{1}, \alpha_{r}\right\}$. Clearly, $e_{\delta} v=0$ for all $\delta \in \Pi \backslash \operatorname{Supp} \sigma$, and we have proved (V2) and (V3). Since $\mathrm{p}_{\mu_{1}}\left(e_{\alpha_{r}} v\right)=$ $e_{-\left(\sigma-\alpha_{r}\right)} v_{\mu_{1}} \neq 0$, we have $v \neq 0$. As $\mathrm{p}_{\mu_{2}}\left(e_{\alpha_{1}} e_{-\sigma} x_{0}\right)=-e_{-\left(\sigma-\alpha_{1}\right)} v_{\mu_{2}} \neq 0$, the vector $v$ is not proportional to $e_{-\sigma} x_{0}$, hence (V1).

Subcase 1.3: $\sigma=\alpha_{1}+2 \alpha_{2}+\ldots+2 \alpha_{r-1}+\alpha_{r}$ with $\operatorname{Supp} \sigma$ of type $\mathrm{C}_{r}(r \geq 3)$. Then

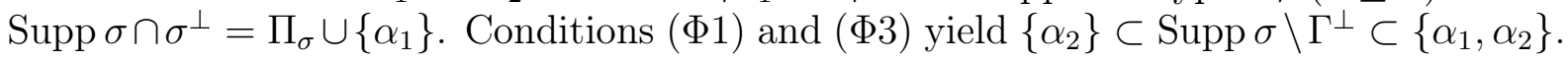
In any case there is $\mu \in \mathrm{E}$ such that $e_{-\left(\sigma-\alpha_{2}\right)} v_{\mu} \neq 0$ and $e_{-\left(\sigma-\alpha_{1}\right)} v_{\mu} \neq 0$. Consider the element

$$
\begin{aligned}
f= & e_{-\left(\sigma-\alpha_{1}\right)} e_{-\alpha_{1}}+e_{-\left(\sigma-\alpha_{1}-\alpha_{2}\right)} e_{-\left(\alpha_{1}+\alpha_{2}\right)}+\ldots+e_{-\left(\sigma-\alpha_{1}-\ldots-\alpha_{r-1}\right)} e_{-\left(\alpha_{1}+\ldots+\alpha_{r-1}\right)}- \\
& e_{-\left(\sigma-\alpha_{2}\right)} e_{-\alpha_{2}}-e_{-\left(\sigma-\alpha_{2}-\alpha_{3}\right)} e_{-\left(\alpha_{2}+\alpha_{3}\right)}-\ldots-e_{-\left(\sigma-\alpha_{2}-\ldots-\alpha_{r-1}\right)} e_{-\left(\alpha_{2}+\ldots+\alpha_{r-1}\right)} \in U(\mathfrak{g}) .
\end{aligned}
$$

Direct computations taking into account (\$3) show that

$$
\begin{aligned}
e_{\alpha_{1}} f v_{\lambda} & =\left\langle\alpha_{1}^{\vee}, \lambda\right\rangle e_{-\left(\sigma-\alpha_{1}\right)} v_{\lambda}, & & e_{\alpha_{1}} e_{-\sigma} v_{\lambda}=-2 e_{-\left(\sigma-\alpha_{1}\right)} v_{\lambda}, \\
e_{\alpha_{2}} f v_{\lambda} & =-\left(\left\langle\alpha_{2}^{\vee}, \lambda\right\rangle+r-1\right) e_{-\left(\sigma-\alpha_{2}\right)} v_{\lambda}, & & e_{\alpha_{2}} e_{-\sigma} v_{\lambda}=-e_{-\left(\sigma-\alpha_{2}\right)} v_{\lambda}, \\
e_{\delta} f v_{\lambda} & =0, & & e_{\delta} e_{-\sigma} v_{\lambda}=0
\end{aligned}
$$

for every $\lambda \in \mathrm{E}$ and $\delta \in \operatorname{Supp} \sigma \backslash\left\{\alpha_{1}, \alpha_{2}\right\}$. We now put

$$
v=\sum_{\lambda \in \mathrm{E}} \frac{2 f+\left\langle\alpha_{1}^{\vee}, \lambda\right\rangle e_{-\sigma}}{\left\langle\alpha_{1}^{\vee}, \lambda\right\rangle+2\left\langle\alpha_{2}^{\vee}, \lambda\right\rangle+2 r-2} v_{\lambda} .
$$

Then $e_{\alpha_{1}} v=0, e_{\alpha_{2}} v=-e_{-\left(\sigma-\alpha_{2}\right)} x_{0}$, and $e_{\delta} v=0$ for all $\delta \in \operatorname{Supp} \sigma \backslash\left\{\alpha_{1}, \alpha_{2}\right\}$. Clearly, $e_{\delta} v=0$ for all $\delta \in \Pi \backslash \operatorname{Supp} \sigma$, and we have proved (V2) and (V3) regardless of whether $\alpha_{1}$ belongs to $\Gamma^{\perp}$ or not. Since $\mathrm{p}_{\mu}\left(e_{\alpha_{2}} v\right)=e_{-\left(\sigma-\alpha_{2}\right)} v_{\mu} \neq 0$, we have $v \neq 0$. As $\mathrm{p}_{\mu}\left(e_{\alpha_{1}} e_{-\sigma} x_{0}\right)=$ $-2 e_{-\left(\sigma-\alpha_{1}\right)} v_{\mu} \neq 0$, the vector $v$ is not proportional to $e_{-\sigma} x_{0}$, hence (V1).

Subcase 1.4: $\sigma=\alpha_{1}+2 \alpha_{2}+3 \alpha_{3}+2 \alpha_{4}$ with $\operatorname{Supp} \sigma$ of type $\mathbf{F}_{4}$. Then $\operatorname{Supp} \sigma \cap \sigma^{\perp}=\Pi_{\sigma}$.

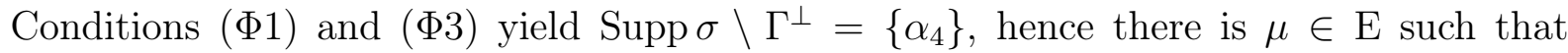
$e_{-\left(\sigma-\alpha_{4}\right)} v_{\mu} \neq 0$ and $e_{-\left(\sigma-\alpha_{3}\right)} v_{\mu} \neq 0$. Consider the element

$$
\begin{gathered}
f=e_{-\left(\sigma-\alpha_{4}\right)} e_{-\alpha_{4}}-e_{-\left(\sigma-\alpha_{3}-\alpha_{4}\right)} e_{-\left(\alpha_{3}+\alpha_{4}\right)}+e_{-\left(\sigma-\alpha_{2}-\alpha_{3}-\alpha_{4}\right)} e_{-\left(\alpha_{2}+\alpha_{3}+\alpha_{4}\right)}- \\
e_{-\left(\sigma-\alpha_{2}-2 \alpha_{3}-\alpha_{4}\right)} e_{-\left(\alpha_{2}+2 \alpha_{3}+\alpha_{4}\right)} \in U(\mathfrak{g}) .
\end{gathered}
$$


Direct computations taking into account $(\Phi 3)$ show that

$$
\begin{array}{ll}
e_{\alpha_{3}} f v_{\lambda}=0, & e_{\alpha_{3}} e_{-\sigma} v_{\lambda}=-2 e_{-\left(\sigma-\alpha_{3}\right)} v_{\lambda}, \\
e_{\alpha_{4}} f v_{\lambda}=\left(\left\langle\alpha_{4}^{\vee}, \lambda\right\rangle+5\right) e_{-\left(\sigma-\alpha_{4}\right)} v_{\lambda}, & e_{\alpha_{4}} e_{-\sigma} v_{\lambda}=-e_{-\left(\sigma-\alpha_{4}\right)} v_{\lambda}, \\
e_{\alpha_{1}} f v_{\lambda}=e_{\alpha_{2}} f v_{\lambda}=0, & e_{\alpha_{1}} e_{-\sigma} v_{\lambda}=e_{\alpha_{2}} e_{-\sigma} v_{\lambda}=0
\end{array}
$$

for every $\lambda \in \mathrm{E}$. We now put

$$
v=\sum_{\lambda \in \mathrm{E}} \frac{f}{\left\langle\alpha_{4}^{\vee}, \lambda\right\rangle+5} v_{\lambda} .
$$

Then $e_{\alpha_{4}} v=e_{-\left(\sigma-\alpha_{4}\right)} x_{0}$ and $e_{\alpha_{1}} v=e_{\alpha_{2}} v=e_{\alpha_{3}} v=0$. Clearly, $e_{\delta} v=0$ for all $\delta \in$ $\Pi \backslash \operatorname{Supp} \sigma$, and we have proved ( (V2) and ( (V3). Since $\mathrm{p}_{\mu}\left(e_{\alpha_{4}} v\right)=e_{-\left(\sigma-\alpha_{4}\right)} v_{\mu} \neq 0$, we have $v \neq 0$. As $\mathrm{p}_{\mu}\left(e_{\alpha_{3}} e_{-\sigma} x_{0}\right)=-2 e_{-\left(\sigma-\alpha_{3}\right)} v_{\mu} \neq 0$, the vector $v$ is not proportional to $e_{-\sigma} x_{0}$, hence (V1).

Subcase 1.5: $\sigma=\alpha_{1}+\alpha_{2}$ with $\operatorname{Supp} \sigma$ of type $\mathrm{G}_{2}$. Then $\operatorname{Supp} \sigma \cap \sigma^{\perp}=\varnothing$. Condition

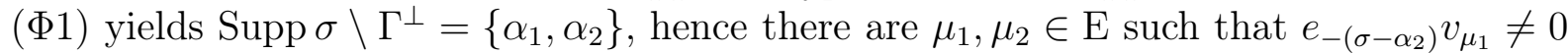
and $e_{-\left(\sigma-\alpha_{1}\right)} v_{\mu_{2}} \neq 0$. Consider the element

$$
f=e_{-\alpha_{2}} e_{-\alpha_{1}} \in U(\mathfrak{g}) .
$$

Direct computations show that

$$
\begin{array}{ll}
e_{\alpha_{1}} f v_{\lambda}=\left\langle\alpha_{1}^{\vee}, \lambda\right\rangle e_{-\left(\sigma-\alpha_{1}\right)} v_{\lambda}, & e_{\alpha_{1}} e_{-\sigma} v_{\lambda}=-3 e_{-\left(\sigma-\alpha_{1}\right)} v_{\lambda}, \\
e_{\alpha_{2}} f v_{\lambda}=\left(\left\langle\alpha_{2}^{\vee}, \lambda\right\rangle+1\right) e_{-\left(\sigma-\alpha_{2}\right)} v_{\lambda}, & e_{\alpha_{2}} e_{-\sigma} v_{\lambda}=e_{-\left(\sigma-\alpha_{2}\right)} v_{\lambda}
\end{array}
$$

for every $\lambda \in \mathrm{E}$. We now put

$$
v=\sum_{\lambda \in \mathrm{E}} \frac{3 f+\left\langle\alpha_{1}^{\vee}, \lambda\right\rangle e_{-\sigma}}{\left\langle\alpha_{1}^{\vee}, \lambda\right\rangle+3\left\langle\alpha_{2}^{\vee}, \lambda\right\rangle+3} v_{\lambda} .
$$

Then $e_{\alpha_{1}} v=0$ and $e_{\alpha_{2}} v=e_{-\left(\sigma-\alpha_{2}\right)} x_{0}$. Clearly, $e_{\delta} v=0$ for all $\delta \in \Pi \backslash \operatorname{Supp} \sigma$, and we have proved ( $(\overline{\mathrm{V} 2})$ and $(\overline{\mathrm{V} 3})$. Since $\mathrm{p}_{\mu_{1}}\left(e_{\alpha_{2}} v\right)=e_{-\left(\sigma-\alpha_{2}\right)} v_{\mu_{1}} \neq 0$, we have $v \neq 0$. As $\mathrm{p}_{\mu_{2}}\left(e_{\alpha_{1}} e_{-\sigma} x_{0}\right)=-3 e_{-\left(\sigma-\alpha_{1}\right)} v_{\mu_{2}} \neq 0$, the vector $v$ is not proportional to $e_{-\sigma} x_{0}$, hence (V1).

Case 2: $\sigma \notin \Delta^{+}$. It follows from Lemma 3.11 that $V_{\sigma}^{\left(T_{\mathrm{ad}}\right)} \cap \mathfrak{g} x_{0}=\{0\}$, hence in this case condition (V1) is equivalent to $v \neq 0$.

Subcase 2.1: $\sigma=2 \alpha$ with $\alpha \in \Pi$. Condition (\$6) yields $\left\langle\alpha^{\vee}, \lambda\right\rangle \in 2 \mathbb{Z}$ for all $\lambda \in \mathrm{E}$. Next, in view of $(\underline{\Phi 1})$ there exists $\mu \in \mathrm{E}$ such that $\left\langle\alpha^{\vee}, \mu\right\rangle>0$. Then the vector

$$
v=\sum_{\lambda \in \mathrm{E}:\left\langle\alpha^{\vee}, \lambda\right\rangle>0} \frac{1}{\left\langle\alpha^{\vee}, \lambda\right\rangle-1} e_{-\alpha} e_{-\alpha} v_{\lambda}
$$

evidently has properties (V1)-(V3).

Subcase 2.2: $\sigma=\alpha+\beta$ for $\alpha, \beta \in \Pi$ with $\alpha \perp \beta$. Condition (\$5) yields $\left\langle\alpha^{\vee}, \lambda\right\rangle=\left\langle\beta^{\vee}, \lambda\right\rangle$ for all $\lambda \in \mathrm{E}$. Next, in view of (\$1) there exists $\mu \in \mathrm{E}$ such that $\left\langle\alpha^{\vee}, \mu\right\rangle>0$. Then the vector

$$
v=\sum_{\lambda \in \mathrm{E}:\left\langle\alpha^{\vee}, \lambda\right\rangle>0} \frac{1}{\left\langle\alpha^{\vee}, \lambda\right\rangle} e_{-\alpha} e_{-\beta} v_{\lambda}
$$

evidently has properties ( $\mathrm{V1})-(\mathrm{V} 3)$. 
Subcase 2.3: $\sigma=\alpha_{1}+2 \alpha_{2}+\alpha_{3}$ with $\operatorname{Supp} \sigma$ of type $\mathrm{A}_{3}$. Then $\operatorname{Supp} \sigma \cap \sigma^{\perp}=\Pi_{\sigma}$. Condition (\$1) yields Supp $\sigma \backslash \Gamma^{\perp}=\left\{\alpha_{2}\right\}$, hence there is $\mu \in \mathrm{E}$ such that $e_{-\left(\sigma-\alpha_{2}\right)} v_{\mu} \neq 0$. Consider the element

$$
f=e_{-\left(\alpha_{1}+\alpha_{2}+\alpha_{3}\right)} e_{-\alpha_{2}}-e_{-\left(\alpha_{1}+\alpha_{2}\right)} e_{-\left(\alpha_{2}+\alpha_{3}\right)} \in U(\mathfrak{g}) .
$$

Direct computations taking into account (\$3) show that

$$
e_{\alpha_{2}} f v_{\lambda}=\left(\left\langle\alpha_{2}^{\vee}, \lambda\right\rangle+1\right) e_{-\left(\sigma-\alpha_{2}\right)} v_{\lambda} \quad \text { and } \quad e_{\alpha_{1}} f v_{\lambda}=e_{\alpha_{3}} f v_{\lambda}=0
$$

for all $\lambda \in \mathrm{E}$. We now put

$$
v=\sum_{\lambda \in \mathrm{E}} \frac{f}{\left\langle\alpha_{2}^{\vee}, \lambda\right\rangle+1} v_{\lambda} .
$$

Then $e_{\alpha_{1}} v=e_{\alpha_{3}} v=0$ and $e_{\alpha_{2}} v=e_{-\left(\sigma-\alpha_{2}\right)} x_{0}$. Clearly, $e_{\delta} v=0$ for all $\delta \in \Pi \backslash \operatorname{Supp} \sigma$, and we have proved ( $(\overline{\mathrm{V} 2})$ and $(\overline{\mathrm{V} 3})$. Since $\mathrm{p}_{\mu}\left(e_{\alpha_{2}} v\right)=e_{-\left(\sigma-\alpha_{2}\right)} v_{\mu} \neq 0$, we have $v \neq 0$, hence (V1).

Subcase 2.4: $\sigma=\alpha_{1}+2 \alpha_{2}+3 \alpha_{3}$ with $\operatorname{Supp} \sigma$ of type $B_{3}$. Then $\operatorname{Supp} \sigma \cap \sigma^{\perp}=\Pi_{\sigma}$. Condition (\$1) yields Supp $\sigma \backslash \Gamma^{\perp}=\left\{\alpha_{3}\right\}$, hence there is $\mu \in \mathrm{E}$ such that $e_{-\left(\sigma-\alpha_{3}\right)} v_{\mu} \neq 0$. Consider the element

$$
f=e_{-\left(\alpha_{1}+2 \alpha_{2}+2 \alpha_{3}\right)} e_{-\alpha_{3}}-e_{-\left(\alpha_{1}+\alpha_{2}+2 \alpha_{3}\right)} e_{-\left(\alpha_{2}+\alpha_{3}\right)}+e_{-\left(\alpha_{1}+\alpha_{2}+\alpha_{3}\right)} e_{-\left(\alpha_{2}+2 \alpha_{3}\right)} \in U(\mathfrak{g}) .
$$

Direct computations taking into account (\$3) show that

$$
e_{\alpha_{3}} f v_{\lambda}=\left(\left\langle\alpha_{3}^{\vee}, \lambda\right\rangle+2\right) e_{-\left(\sigma-\alpha_{3}\right)} v_{\lambda} \quad \text { and } \quad e_{\alpha_{1}} f v_{\lambda}=e_{\alpha_{2}} f v_{\lambda}=0
$$

for all $\lambda \in \mathrm{E}$. We now put

$$
v=\sum_{\lambda \in \mathrm{E}} \frac{f}{\left\langle\alpha_{3}^{\vee}, \lambda\right\rangle+2} v_{\lambda} .
$$

Then $e_{\alpha_{3}} v=e_{-\left(\sigma-\alpha_{3}\right)} x_{0}$ and $e_{\alpha_{1}} v=e_{\alpha_{2}} v=0$. Clearly, $e_{\delta} v=0$ for all $\delta \in \Pi \backslash \operatorname{Supp} \sigma$, and we have proved $(\underline{\mathrm{V} 2})$ and $(\underline{\mathrm{V} 3})$. Since $\mathrm{p}_{\mu}\left(e_{\alpha_{3}} v\right)=e_{-\left(\sigma-\alpha_{3}\right)} v_{\mu} \neq 0$, we have $v \neq 0$, hence (V1).

Subcase 2.5: $\sigma=2 \alpha_{1}+\ldots+2 \alpha_{r}$ with $\operatorname{Supp} \sigma$ of type $\mathrm{B}_{r}(r \geq 2)$. Then $\operatorname{Supp} \sigma \cap \sigma^{\perp}=\Pi_{\sigma}$. Condition (\$1) yields Supp $\sigma \backslash \Gamma^{\perp}=\left\{\alpha_{1}\right\}$, hence there is $\mu \in \mathrm{E}$ such that $e_{-\left(\sigma-\alpha_{1}\right)} v_{\mu} \neq 0$. Consider the element

$$
\begin{array}{r}
f=4 e_{-\sigma+\alpha_{1}} e_{-\alpha_{1}}+4 e_{-\sigma+\alpha_{1}+\alpha_{2}} e_{-\left(\alpha_{1}+\alpha_{2}\right)}+\ldots+4 e_{-\sigma+\alpha_{1}+\ldots+\alpha_{r-1}} e_{-\left(\alpha_{1}+\ldots+\alpha_{r-1}\right)}+ \\
e_{-\left(\alpha_{1}+\ldots+\alpha_{r}\right)} e_{-\left(\alpha_{1}+\ldots+\alpha_{r}\right)} \in U(\mathfrak{g}) .
\end{array}
$$

Direct computations taking into account (\$3) show that

$$
e_{\alpha_{1}} f v_{\lambda}=\left(4\left\langle\alpha_{1}^{\vee}, \lambda\right\rangle+4 r-6\right) v_{\lambda} \quad \text { and } \quad e_{\delta} f v_{\lambda}=0
$$

for all $\lambda \in \operatorname{E}$ and $\delta \in \operatorname{Supp} \sigma \backslash\left\{\alpha_{1}\right\}$. We now put

$$
v=\sum_{\lambda \in \mathrm{E}} \frac{f}{4\left\langle\alpha_{1}^{\vee}, \lambda\right\rangle+4 r-6} v_{\lambda} .
$$

Then $e_{\alpha_{1}} v=e_{-\left(\sigma-\alpha_{1}\right)} x_{0}$ and $e_{\delta} v=0$ for all $\delta \in \operatorname{Supp} \sigma \backslash\left\{\alpha_{1}\right\}$. Clearly, $e_{\delta} v=0$ for all $\delta \in \Pi \backslash \operatorname{Supp} \sigma$, and we have proved (V2) and (3). Since $\mathrm{p}_{\mu}\left(e_{\alpha_{1}} v\right)=e_{-\left(\sigma-\alpha_{1}\right)} v_{\mu} \neq 0$, we have $v \neq 0$, hence (V1). 
Subcase 2.6: $\sigma=2 \alpha_{1}+\ldots+2 \alpha_{r-2}+\alpha_{r-1}+\alpha_{r}$ with Supp $\sigma$ of type $\mathrm{D}_{r}(r \geq 4)$. Then Supp $\sigma \cap \sigma^{\perp}=\Pi_{\sigma}$. Condition (\$1) yields $\operatorname{Supp} \sigma \backslash \Gamma^{\perp}=\left\{\alpha_{1}\right\}$, hence there is $\mu \in$ E such that $e_{-\left(\sigma-\alpha_{1}\right)} v_{\mu} \neq 0$. Consider the element

$$
f=e_{-\sigma+\alpha_{1}} e_{-\alpha_{1}}+e_{-\sigma+\alpha_{1}+\alpha_{2}} e_{-\left(\alpha_{1}+\alpha_{2}\right)}+\ldots+e_{-\sigma+\alpha_{1}+\ldots+\alpha_{r-1}} e_{-\left(\alpha_{1}+\ldots+\alpha_{r-1}\right)} \in U(\mathfrak{g}) .
$$

Direct computations taking into account (\$3) show that

$$
e_{\alpha_{1}} f v_{\lambda}=\left(\left\langle\alpha_{1}^{\vee}, \lambda\right\rangle+r-2\right) e_{-\sigma+\alpha_{1}} v_{\lambda} \quad \text { and } \quad e_{\delta} f v_{\lambda}=0
$$

for all $\lambda \in \operatorname{E}$ and $\delta \in \operatorname{Supp} \sigma \backslash\left\{\alpha_{1}\right\}$. We now put

$$
v=\sum_{\lambda \in \mathrm{E}} \frac{f}{\left\langle\alpha_{1}^{\vee}, \lambda\right\rangle+r-2} v_{\lambda} .
$$

Then $e_{\alpha_{1}} v=e_{-\left(\sigma-\alpha_{1}\right)} x_{0}$ and $e_{\delta} v=0$ for all $\delta \in \operatorname{Supp} \sigma \backslash\left\{\alpha_{1}\right\}$. Clearly, $e_{\delta} v=0$ for all $\delta \in \Pi \backslash \operatorname{Supp} \sigma$, and we have proved (V2) and (V3). Since $\mathrm{p}_{\mu}\left(e_{\alpha_{1}} v\right)=e_{-\left(\sigma-\alpha_{1}\right)} v_{\mu} \neq 0$, we have $v \neq 0$, hence ( $\mathrm{V} 1)$.

Subcase 2.7: $\sigma=4 \alpha_{1}+2 \alpha_{2}$ with $\operatorname{Supp} \sigma$ of type $\mathrm{G}_{2}$. Then Supp $\sigma \cap \sigma^{\perp}=\Pi_{\sigma}$. Condition (\$1) yields $\operatorname{Supp} \sigma \backslash \Gamma^{\perp}=\left\{\alpha_{1}\right\}$, hence there is $\mu \in \mathrm{E}$ such that $e_{-\left(\sigma-\alpha_{1}\right)} v_{\mu} \neq 0$. Consider the element

$$
f=4 e_{-\sigma+\alpha_{1}} e_{-\alpha_{1}}+4 e_{-\sigma+\alpha_{1}+\alpha_{2}} e_{-\left(\alpha_{1}+\alpha_{2}\right)}-3 e_{-\left(2 \alpha_{1}+\alpha_{2}\right)} e_{-\left(2 \alpha_{1}+\alpha_{2}\right)} \in U(\mathfrak{g}) .
$$

Direct computations taking into account (\$3) show that

$$
e_{\alpha_{1}} f v_{\lambda}=\left(4\left\langle\alpha_{1}^{\vee}, \lambda\right\rangle+18\right) e_{-\sigma+\alpha_{1}} \quad \text { and } \quad e_{\alpha_{2}} f v_{\lambda}=0
$$

for all $\lambda \in \mathrm{E}$. We now put

$$
v=\sum_{\lambda \in \mathrm{E}} \frac{f}{4\left\langle\alpha_{1}^{\vee}, \lambda\right\rangle+18} v_{\lambda} .
$$

Then $e_{\alpha_{1}} v=e_{-\left(\sigma-\alpha_{1}\right)} x_{0}$ and $e_{\alpha_{2}} v=0$. Clearly, $e_{\delta} v=0$ for all $\delta \in \Pi \backslash \operatorname{Supp} \sigma$, and we have proved ( $(\mathrm{V} 2)$ and $(\overline{\mathrm{V} 3})$. Since $\mathrm{p}_{\mu}\left(e_{\alpha_{1}} v\right)=e_{-\left(\sigma-\alpha_{1}\right)} v_{\mu} \neq 0$, we have $v \neq 0$, hence (V1).

The proof of Proposition 3.38 is completed.

\section{Applications}

Given a finitely generated and saturated monoid $\Gamma \subset \Lambda^{+}$, recall the set $\Phi(\Gamma)$ and Theorem 3.1 from $\S 3.1$. All results obtained in this section depend only on the following parts of Theorem 3.1

- the $T_{\text {ad }}$-module $T_{X_{0}} \mathrm{M}_{\Gamma}$ is multiplicity-free;

- every element of $\Phi(\Gamma)$ satisfies conditions $(\Phi 1)-(\Phi 8)$.

We point out that the existence part of Theorem 3.1 (see Proposition 3.38) is not used in this section. 
4.1. Auxiliary results on $\Phi(\Gamma)$. Throughout this subsection, $\Gamma \subset \Lambda^{+}$is an arbitrary finitely generated and saturated monoid.

Lemma 4.1. The set $\Phi(\Gamma)$ contains no proportional elements.

Proof. Assume that $\sigma, \sigma^{\prime} \in \Phi(\Gamma)$ are two distinct proportional elements. Thanks to ( $\Phi 2$ ), one has $\sigma, \sigma^{\prime} \in \bar{\Sigma}(G)$. By inspecting Table 1, we get the only two following possibilities (up to interchanging $\sigma$ and $\sigma^{\prime}$ ).

Case 1: $\sigma=\alpha$ for some $\alpha \in \Pi$ and $\sigma^{\prime}=2 \alpha$. Then condition (\$8) for $\sigma$ contradicts condition (\$7) for $\sigma^{\prime}$.

Case 2: $\sigma=\alpha_{1}+\ldots+\alpha_{r}$ with $\operatorname{Supp} \sigma$ of type $\mathrm{B}_{r}(r \geq 2)$ and $\sigma^{\prime}=2 \sigma$. Then

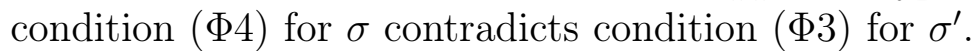

Lemma 4.2. If $\alpha+\beta \in \Phi(\Gamma)$ for some $\alpha, \beta \in \Pi$ with $\alpha \perp \beta$, then $\Phi(\Gamma) \cap\{\alpha, \beta\}=\varnothing$.

Proof. Assume without loss of generality that $\alpha \in \Phi(\Gamma)$. Then $\alpha \in \mathbb{Z} \Gamma$ by ( $\Phi 1$ ). On the other hand, $2=\left\langle\alpha^{\vee}, \alpha\right\rangle \neq\left\langle\beta^{\vee}, \alpha\right\rangle=0$, which contradicts condition (\$5) for $\alpha+\beta$.

Lemma 4.3. Suppose that $\sigma \in \Phi(\Gamma) \backslash \Pi, \alpha \in \operatorname{Supp} \sigma$, and $\left\langle\alpha^{\vee}, \sigma\right\rangle>0$. Then $\alpha \notin \Phi(\Gamma)$.

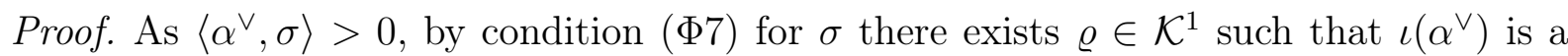

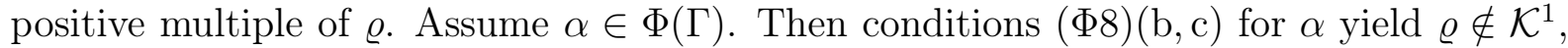
a contradiction.

Corollary 4.4. Suppose that $\sigma \in \Phi(\Gamma) \backslash \Pi$. Then there exists $\alpha \in \operatorname{Supp} \sigma$ such that $\alpha \notin \Phi(\Gamma)$.

Proof. This follows from Lemmas 4.3 and 3.20 .

Proposition 4.5. Every element of $\Phi(\Gamma)$ is primitive in the lattice $\mathbb{Z} \Phi(\Gamma)$.

Proof. Assume there exists an element $\sigma \in \mathbb{Z} \Phi(\Gamma)$ such that $n \sigma \in \Phi(\Gamma)$ for some $n \geq 2$. Since $\Phi(\Gamma) \subset \bar{\Sigma}(G) \subset \mathbb{Z}^{+} \Pi$, it follows that $\sigma \in \mathbb{Z}^{+} \Pi$. An inspection of Table 1 shows that $n=2$ and one of the three cases below occurs.

Case 1: $\sigma=\alpha \in \Pi$. Then $2 \alpha \in \Phi(\Gamma)$ and condition (\$6) yields

$$
\left\langle\alpha^{\vee}, \tau\right\rangle \in 2 \mathbb{Z} \text { for all } \tau \in \Phi(\Gamma) .
$$

Since $\sigma \in \mathbb{Z} \Phi(\Gamma)$, there exists $\sigma_{1} \in \Phi(\Gamma) \backslash\{2 \alpha\}$ such that $\alpha \in \operatorname{Supp} \sigma_{1}$. Then $\Pi_{\sigma_{1}} \subset \alpha^{\perp}$ by (\$3) . A case-by-case check of all entries in Table 1 together with (4.1) and Lemma 4.2 yields only the following two possibilities for $\sigma_{1}$ and $\alpha$ :

- $\sigma_{1}=\alpha_{1}+\alpha_{2}$ with Supp $\sigma_{1}$ of type $\mathbf{B}_{2}$ and $\alpha=\alpha_{2}$;

- $\sigma_{1}=\alpha_{1}+2 \alpha_{2}+2 \alpha_{3}+\ldots+2 \alpha_{r-1}+\alpha_{r}$ with $\operatorname{Supp} \sigma_{1}$ of type $C_{r}(r \geq 3)$ and $\alpha=\alpha_{1}$.

It is easy to see that $\sigma_{1}$ is the unique element in $\Phi(\Gamma) \backslash\{2 \alpha\}$ with $\alpha \in \operatorname{Supp} \sigma_{1}$. The subsequent consideration is divided into three subcases.

Subcase 1.1: $\sigma_{1}=\alpha_{1}+\alpha_{2}$ with $\operatorname{Supp} \sigma_{1}$ of type $\mathrm{B}_{2}$ and $\alpha=\alpha_{2}$. Then there exists $\sigma_{2} \in \Phi(\Gamma) \backslash\left\{2 \alpha, \sigma_{1}\right\}$ such that $\alpha_{1} \in \operatorname{Supp} \sigma_{2}$. Recall that $\alpha_{2} \notin \operatorname{Supp} \sigma_{2}$. As $\left\langle\alpha_{1}^{\vee}, \sigma_{1}\right\rangle=1$, one has $\sigma_{2} \neq \alpha_{1}$ by Lemma 4.3 and $\sigma_{2} \neq 2 \alpha_{1}$ by condition (\$6). Further, $\sigma_{2} \neq \alpha_{1}+\beta$ for all $\beta \in \Pi$ with $\alpha_{1} \perp \beta$; otherwise one would have $\left\langle\alpha_{1}^{\vee}, \sigma_{1}\right\rangle>0$ and $\left\langle\beta^{\vee}, \sigma_{1}\right\rangle \leq 0$, contradicting

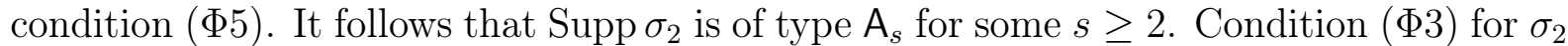
yields $\Pi_{\sigma_{2}} \subset \sigma_{1}^{\perp}$, whence $s=2$ and $\sigma_{2}=\alpha_{1}+\beta_{1}$ for some $\beta_{1} \in \Pi \backslash\left\{\alpha_{1}, \alpha_{2}\right\}$. Note that $\sigma_{2}$ is the unique element in $\Phi(\Gamma) \backslash\left\{2 \alpha, \sigma_{1}\right\}$ with $\alpha_{1} \in \operatorname{Supp} \sigma_{2}$. Iterating the above argument 
leads to an infinite chain $\sigma_{3}, \sigma_{4}, \ldots \subset \Phi(\Gamma)$ such that for every $i \geq 3$ the following properties hold:

- $\operatorname{Supp} \sigma_{i}$ is of type $\mathrm{A}_{2}$;

- $\sigma_{i}=\beta_{i-2}+\beta_{i-1}$ for some $\beta_{i-1} \in \Pi \backslash\left\{\alpha_{1}, \alpha_{2}, \beta_{1}, \ldots, \beta_{i-2}\right\}$;

- $\sigma_{i}$ is the unique element in $\Phi(\Gamma) \backslash\left\{2 \alpha, \sigma_{1}, \ldots, \sigma_{i-1}\right\}$ with $\beta_{i-2} \in \operatorname{Supp} \sigma_{i}$.

As $\Phi(\Gamma)$ is finite, we have got a contradiction.

Subcase 1.2: $\sigma_{1}=\alpha_{1}+2 \alpha_{2}+\alpha_{3}$ with $\operatorname{Supp} \sigma_{1}$ of type $C_{3}$ and $\alpha=\alpha_{1}$. Then there exists $\sigma_{2} \in \Phi(\Gamma) \backslash\left\{2 \alpha, \sigma_{1}\right\}$ such that $\alpha_{2} \in \operatorname{Supp} \sigma_{2}$. Recall that $\alpha_{1} \notin \operatorname{Supp} \sigma_{2}$. As $\sigma_{2} \in \mathbb{Z} \Gamma$

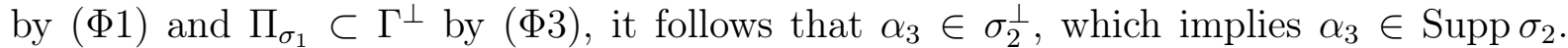
If $\operatorname{Supp} \sigma_{2}=\left\{\alpha_{2}, \alpha_{3}\right\}$ then $\sigma_{2}=k\left(\alpha_{2}+\alpha_{3}\right)$ with $k \in\{1,2\}$, hence $\left\langle\alpha_{3}^{\vee}, \sigma_{2}\right\rangle \neq 0$, which contradicts condition (\$3) for $\sigma_{1}$. Consequently, $\operatorname{Supp} \sigma_{2} \neq\left\{\alpha_{2}, \alpha_{3}\right\}$ and there exists $\alpha_{4} \in \Pi \backslash\left\{\alpha_{1}, \alpha_{2}, \alpha_{3}\right\}$ such that $\Pi \backslash \alpha_{4}^{\perp}=\left\{\alpha_{3}, \alpha_{4}\right\}$, the set $\left\{\alpha_{1}, \alpha_{2}, \alpha_{3}, \alpha_{4}\right\}$ is of type $\mathrm{F}_{4}$, and $\operatorname{Supp} \sigma_{2}=\left\{\alpha_{2}, \alpha_{3}, \alpha_{4}\right\}$. It follows that

$$
\sigma_{2} \in\left\{\alpha_{2}+\alpha_{3}+\alpha_{4}, 2 \alpha_{2}+2 \alpha_{3}+2 \alpha_{4}, 3 \alpha_{2}+2 \alpha_{3}+\alpha_{4}\right\}
$$

Condition (4.1) leaves the only possibility $\sigma_{2}=2 \alpha_{2}+2 \alpha_{3}+2 \alpha_{4}$. Condition (\$3) for $\sigma_{2}$ then implies $\alpha_{2} \in \Gamma^{\perp}$, which is false because $\left\langle\alpha_{2}^{\vee}, \sigma_{1}\right\rangle \neq 0$.

Subcase 1.3: $\sigma_{1}=\alpha_{1}+2 \alpha_{2}+2 \alpha_{3}+\ldots+2 \alpha_{r-1}+\alpha_{r}$ with $\operatorname{Supp} \sigma_{1}$ of type $C_{r}(r \geq 4)$; $\alpha=\alpha_{1}$. Then there exists $\sigma_{2} \in \Phi(\Gamma) \backslash\left\{2 \alpha, \sigma_{1}\right\}$ such that $\alpha_{2} \in \operatorname{Supp} \sigma_{2}$. Recall that

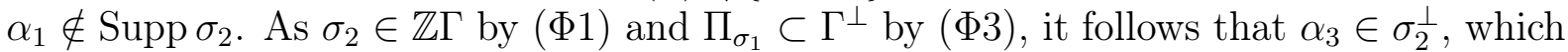
implies $\alpha_{3} \in \operatorname{Supp} \sigma_{2}$. Iterating this argument yields $\alpha_{4}, \ldots, \alpha_{r} \in \operatorname{Supp} \sigma_{2}$. It follows that Supp $\sigma_{2}=\left\{\alpha_{2}, \ldots, \alpha_{r}\right\}$, so that Supp $\sigma_{2}$ is of type $C_{r-1}$ and $\sigma_{2}=\alpha_{2}+2 \alpha_{3}+\ldots+2 \alpha_{r-1}+\alpha_{r}$. Since $\alpha_{3} \in \Pi_{\sigma_{1}}$ and $\left\langle\alpha_{3}^{\vee}, \sigma_{2}\right\rangle \neq 0$, we obtain a contradiction with condition (\$3) for $\sigma_{1}$.

Case 2: $\sigma=\alpha_{1}+\ldots+\alpha_{r}$ with $\operatorname{Supp} \sigma$ of type $\mathrm{B}_{r}(r \geq 2)$. Then $2 \sigma=2 \alpha_{1}+\ldots+2 \alpha_{r} \in$ $\Phi(\Gamma)$ and hence $\alpha_{2}, \ldots, \alpha_{r} \in \Gamma^{\perp}$ by $(\underline{\Phi 3})$. In view of condition $\sigma \in \mathbb{Z} \Phi(\Gamma)$ there exists $\tau \in \Phi(\Gamma) \backslash\{2 \sigma\}$ such that $\alpha_{1} \in \operatorname{Supp} \tau$. As $\tau \in \mathbb{Z} \Gamma$ by $(\Phi 1)$, condition $\alpha_{2} \in \tau^{\perp}$ implies $\alpha_{2} \in \operatorname{Supp} \tau$. Iterating this argument yields $\alpha_{3}, \ldots, \alpha_{r} \in \operatorname{Supp} \tau$, therefore $\operatorname{Supp} \sigma \subset$ Supp $\tau$. Since $\Phi(\Gamma) \subset \bar{\Sigma}(G)$, an inspection of Table 1 shows that conditions $\alpha_{1} \notin \Pi_{\tau}$ and $\alpha_{r} \in \tau^{\perp}$ cannot hold for an element $\tau \in \Phi(\Gamma) \backslash\{2 \sigma\}$, a contradiction.

Case 3: $\sigma=2 \alpha_{1}+\alpha_{2}$ with $\operatorname{Supp} \sigma$ of type $\mathrm{G}_{2}$. Then $2 \sigma=4 \alpha_{1}+2 \alpha_{2} \in \Phi(\Gamma)$ and hence $\alpha_{2} \in \Gamma^{\perp}$ by (\$3). Since $\Phi(\Gamma) \subset \bar{\Sigma}(G)$, an inspection of Table 1 yields that there are no elements $\tau \in \Phi(\Gamma) \backslash\{2 \sigma\}$ such that $\alpha_{2} \in \operatorname{Supp} \tau$ and $\alpha_{2} \in \tau^{\perp}$, which contradicts the condition $\sigma \in \mathbb{Z} \Phi(\Gamma)$.

The following proposition is similar to [BVS16, Proposition 5.4].

Proposition 4.6. Every $\sigma \in \Phi(\Gamma)$ satisfies the condition $\sigma \notin \mathbb{Z}^{+}(\Phi(\Gamma) \backslash\{\sigma\})$.

Proof. Fix an arbitrary $\sigma \in \Phi(\Gamma)$ and assume that $\sigma \in \mathbb{Z}^{+}(\Phi(\Gamma) \backslash\{\sigma\})$. Fix a subset $\Phi_{\sigma} \subset \Phi(\Gamma) \backslash\{\sigma\}$ such that $\sigma=\sum_{\tau \in \Phi_{\sigma}} n_{\tau} \tau$ with all the coefficients $n_{\tau}$ being positive integers.

Then Lemma 4.1 yields

$$
\left|\Phi_{\sigma}\right| \geq 2
$$

Clearly, every $\tau \in \Phi_{\sigma}$ satisfies 
moreover,

$$
\Pi_{\sigma} \subset \tau^{\perp}
$$

by (\$3). Assume that $\left|\operatorname{Supp} \sigma \backslash \Pi_{\sigma}\right|=1$. As $\Pi_{\sigma} \subset \sigma^{\perp}$, for each $\tau \in \Phi_{\sigma}$ condition (4.4) implies that $\tau$ is proportional to $\sigma$, which is impossible by Lemma 4.1. Thus in what follows we assume that $\left|\operatorname{Supp} \sigma \backslash \Pi_{\sigma}\right| \geq 2$. Then an inspection of Table 1 leaves the following five cases.

Case 1: $\sigma=\alpha_{1}+\alpha_{2}$ with $\operatorname{Supp} \sigma$ of type $\mathrm{A}_{2}, \mathrm{~B}_{2}$, or $\mathrm{G}_{2}$. Condition (4.2) yields $\Phi_{\sigma}=\left\{\alpha_{1}, \alpha_{2}\right\}$, which contradicts Corollary 4.4.

Case 2: $\sigma=\alpha+\beta$ for some $\alpha, \beta \in \Pi$ with $\alpha \perp \beta$. Condition (4.2) yields $\Phi_{\sigma}=\{\alpha, \beta\}$, which contradicts Lemma 4.2 .

Case 3: $\sigma=\alpha_{1}+\ldots+\alpha_{r}$ with $\operatorname{Supp} \sigma$ of type $\mathrm{A}_{r}(r \geq 3)$. An easy computation based on conditions (4.3) and (4.4) shows that every element $\tau \in \Phi_{\sigma}$ has the form

$$
\begin{aligned}
& \tau=k \alpha_{1}+(k+d) \alpha_{2}+\ldots+(k+(r-1) d) \alpha_{r} \\
& \quad \text { for some } k, d \in \mathbb{Z} \text { with } k \geq 0, k+(r-1) d \geq 0 .
\end{aligned}
$$

Since $r \geq 3$ and $\Phi_{\sigma} \subset \bar{\Sigma}(G)$, an inspection of Table 1 yields $\Phi_{\sigma}=\varnothing$, which contradicts condition (4.2).

Case 4: $\sigma=\alpha_{1}+\ldots+\alpha_{r}$ with $\operatorname{Supp} \sigma$ of type $\mathrm{B}_{r}(r \geq 3)$. The same computation as in Case 3 shows that every element $\tau \in \Phi_{\sigma}$ has the form (4.5). Since $r \geq 3$ and $\Phi_{\sigma} \subset \bar{\Sigma}(G)$, an inspection of Table 1 yields $\Phi_{\sigma}=\varnothing$ for $r \geq 5$ and $\Phi_{\sigma} \subset\left\{\alpha_{r-2}+2 \alpha_{r-1}+3 \alpha_{r}\right\}$ for $r \in\{3,4\}$. In any case we obtain a contradiction with (4.2).

Case 5: $\sigma=\alpha_{1}+2 \alpha_{2}+2 \alpha_{3}+\ldots+2 \alpha_{r-1}+\alpha_{r}$ with $\operatorname{Supp} \sigma$ of type $\mathrm{C}_{r}(r \geq 3)$. It follows from conditions (4.3) and (4.4) that every element $\tau \in \Phi_{\sigma}$ has the form

$$
\tau=k_{1} \alpha_{1}+k_{2}\left(2 \alpha_{2}+2 \alpha_{3}+\ldots+2 \alpha_{r-1}+\alpha_{r}\right)
$$

for some non-negative integers $k_{1}, k_{2}$. Since $r \geq 3$ and $\Phi_{\sigma} \subset \bar{\Sigma}(G)$, an inspection of Table 1 yields $\Phi_{\sigma} \subset\left\{\alpha_{1}\right\}$, which contradicts condition (4.2).

Lemma 4.7. Suppose that $\alpha \in \mathbb{Z} \Gamma \cap \Pi$ and $2 \alpha \in \Phi(\Gamma)$. Then $\alpha$ is primitive in $\mathbb{Z} \Gamma$.

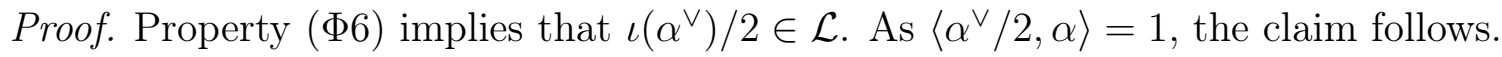

Remark 4.8. Lemma 4.1, Proposition 4.5, and Proposition 4.6 would follow easily if we knew a priori that the set $\Phi(\Gamma)$ is linearly independent.

The above remark leads to the following natural question.

Question 4.9. Is the set $\Phi(\Gamma)$ linearly independent?

4.2. Applications to affine spherical $G$-varieties. Let $X$ be an affine spherical $G$ variety. Consider the corresponding $T_{\text {ad }}$-orbit closure $C_{X} \subset \mathrm{M}_{\Gamma_{X}}$ (see §2.7) and equip it with its reduced subscheme structure. Recall the root monoid $\Xi_{X}$ from Definition 2.12 ,

Proposition 4.10. Suppose that $\sigma$ is an indecomposable element of $\Xi_{X}$. Then

(a) $\sigma \in \Phi\left(\Gamma_{X}\right)$;

(b) $\sigma$ is primitive in the lattice $\mathbb{Z}_{X}$. 
Proof. (国) This follows readily from Corollary 2.24 together with the inclusion $T_{X_{0}} C_{X} \subset$ $T_{X_{0}} \mathrm{M}_{\Gamma_{X}}$.

(b) Part (国) yields $\mathbb{Z} \Xi_{X} \subset \mathbb{Z} \Phi\left(\Gamma_{X}\right)$, which implies the required result in view of Proposition 4.5.

Recall the monoid $\Xi_{X}^{\text {sat }}$ and the set $\bar{\Sigma}_{X}$ defined in $\S 2.3$.

Theorem 4.11. There is an inclusion $\bar{\Sigma}_{X} \subset \Xi_{X}$. In particular, $\Xi_{X}=\Xi_{X}^{\text {sat }}$ and the monoid $\Xi_{X}$ is free.

Proof. Take any $\sigma \in \bar{\Sigma}_{X}$. Since $\mathbb{Z}^{+} \bar{\Sigma}_{X}=\Xi_{X}^{\text {sat }}$ and the set $\bar{\Sigma}_{X}$ is linearly independent, there exists a positive integer $n$ such that $n \sigma$ is an indecomposable element of $\Xi_{X}$. It follows from Proposition 4.10(b) that $n=1$ and hence $\sigma \in \Xi_{X}$.

Corollary 4.12. There is an inclusion $\bar{\Sigma}_{X} \subset \Phi\left(\Gamma_{X}\right)$.

Proof. This follows from Theorem 4.11 and Proposition 4.10(国).

Corollary 4.13. The $T_{\mathrm{ad}}$-orbit closure $C_{X} \subset \mathrm{M}_{\Gamma_{X}}$ is an affine space of dimension $\left|\bar{\Sigma}_{X}\right|$.

Proof. Combining Theorems 2.23 and 4.11 we find that $C_{X}$ is a multiplicity-free affine $T_{\text {ad }}$-variety whose weight monoid is generated by the linearly independent set $\bar{\Sigma}_{X}$. All the claims follow readily.

Corollary 4.14. Let $\Gamma \subset \Lambda^{+}$be a finitely generated and saturated monoid. Then every $T_{\text {ad-orbit closure in }} \mathrm{M}_{\Gamma}$, equipped with its reduced subscheme structure, is an affine space.

Proof. This follows from Theorem 2.20 and Corollary 4.13.

Theorem 4.15. Up to a $G$-isomorphism, every affine spherical $G$-variety $X$ is uniquely determined by the pair $\left(\Gamma_{X}, \bar{\Sigma}_{X}\right)$.

Proof. Let $X_{1}, X_{2}$ be two affine spherical $G$-varieties with $\Gamma_{X_{1}}=\Gamma_{X_{2}}$ and $\bar{\Sigma}_{X_{1}}=\bar{\Sigma}_{X_{2}}$ and assume that $X_{1}, X_{2}$ are not $G$-isomorphic. Put $\Gamma=\Gamma_{X_{1}}=\Gamma_{X_{2}}$ and $\bar{\Sigma}=\bar{\Sigma}_{X_{1}}=\bar{\Sigma}_{X_{2}}$ for brevity. Consider the closed subsets $C_{X_{1}}, C_{X_{2}}$, and $Z=C_{X_{1}} \cup C_{X_{2}}$ in $\mathrm{M}_{\Gamma}$ and equip each of them with its reduced subscheme structure. Thanks to Corollary 4.13,

$$
\operatorname{dim} C_{X_{1}}=\operatorname{dim} C_{X_{2}}=\operatorname{dim} Z=|\bar{\Sigma}| .
$$

It follows from Theorem 2.20 that $C_{X_{1}} \neq C_{X_{2}}$, hence $C_{X_{1}}$ and $C_{X_{2}}$ are distinct irreducible components of $Z$. Consequently, $X_{0}$ is a singular point of $Z$, which implies

$$
\operatorname{dim} T_{X_{0}} Z \geq|\bar{\Sigma}|+1 .
$$

By Theorems 2.23 and 4.11, $C_{X_{1}}$ and $C_{X_{2}}$ are isomorphic multiplicity-free affine $T_{\mathrm{ad}^{-}}$ varieties with weight monoid $\mathbb{Z}^{+} \bar{\Sigma}$, therefore all $T_{\text {ad }}$-weights of the algebra $\mathbb{k}[Z]$ belong to $\mathbb{Z}^{+} \bar{\Sigma}$. In particular,

$$
\left\{\tau \in \mathfrak{X}\left(T_{\text {ad }}\right) \mid-\tau \text { is a } T_{\text {ad }} \text {-weight of } T_{X_{0}} Z\right\} \subset \mathbb{Z}^{+} \bar{\Sigma} .
$$

Since $T_{X_{0}} Z \subset T_{X_{0}} \mathrm{M}_{\Gamma}$ and $T_{X_{0}} \mathrm{M}_{\Gamma}$ is a multiplicity-free $T_{\text {ad }}$-module by Theorem 3.1, inequality (4.6) implies that the set $\Phi(\Gamma) \backslash \bar{\Sigma}$ contains an element that belongs to $\mathbb{Z}^{+} \bar{\Sigma}$. The latter is impossible by Proposition 4.6. 
Recall from $\S 2.3$ that to every affine spherical $G$-variety $X$ one assigns the set $\Sigma_{X}$ of spherical roots of $X$.

The following result, which strengthens Theorem 4.15, was first obtained by Losev in [Lo09b, Theorem 1.2].

Corollary 4.16. Up to a $G$-isomorphism, every affine spherical $G$-variety $X$ is uniquely determined by the pair $\left(\Gamma_{X}, \Sigma_{X}\right)$.

Proof. Thanks to Corollary 4.12 and Proposition 4.5 , the set $\bar{\Sigma}_{X}$ is uniquely determined by the pair $\left(\Gamma_{X}, \Sigma_{X}\right)$ as the set of primitive elements $\nu$ of the lattice $\mathbb{Z} \Phi\left(\Gamma_{X}\right)$ such that $\mathbb{Q}^{+} \nu$ is an extremal ray of the cone $\mathbb{Q}^{+} \Sigma_{X} \subset \mathbb{Z} \Gamma_{X} \otimes_{\mathbb{Z}} \mathbb{Q}$. It remains to apply Theorem 4.15.

The following corollary is a particular case of Corollary 4.23 below, which was first obtained in [AB05, Corollary 3.4].

Corollary 4.17. Up to a G-isomorphism, there are only finitely many affine spherical $G$-varieties with a prescribed weight monoid.

Proof. Let $X$ be an affine spherical $G$-variety. Combining Corollary 4.12 with condition (\$2) yields $\bar{\Sigma}_{X} \subset \bar{\Sigma}(G)$. As the set $\bar{\Sigma}(G)$ is finite, the claim follows from Theorem 4.15 ,

Corollary 4.18. Suppose that $\Gamma \subset \Lambda^{+}$is a finitely generated and saturated monoid. Then every irreducible component of $\mathrm{M}_{\Gamma}$, equipped with its reduced subscheme structure, is an affine space.

Proof. It follows from Theorem 2.20 and Corollary 4.17 that every irreducible component

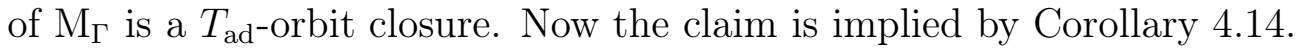

Let $X$ be an affine spherical $G$-variety. For every $\sigma \in \Sigma_{X}$, let $\bar{\sigma}$ denote the unique element in the set $\mathbb{Z}^{+} \sigma \cap \bar{\Sigma}_{X}$. The following result is a version of [Lo09a, Theorem 2] for affine spherical $G$-varieties.

Theorem 4.19. Under the above assumptions, $\bar{\sigma} \in\{\sigma, 2 \sigma\}$ for every $\sigma \in \Sigma_{X}$. Moreover, $\bar{\sigma}=2 \sigma$ if and only if one of the following cases occurs:

(1) $\sigma \notin \bar{\Sigma}(G)$;

(2) $\sigma=\alpha \in \Pi$ and $\mathbb{Q}^{+} \iota\left(\alpha^{\vee}\right)$ is an extremal ray of the cone $\mathcal{K}$;

(3) $\sigma=\alpha_{1}+\ldots+\alpha_{r}$ with $\operatorname{Supp} \sigma$ of type $\mathrm{B}_{r}(r \geq 2)$ and $\alpha_{r} \in \Gamma_{X}^{\perp}$.

Proof. Fix any $\sigma \in \Sigma_{X}$. Corollary 4.12 yields $\bar{\sigma} \in \Phi\left(\Gamma_{X}\right)$, which together with Lemma 4.1 implies that $\bar{\sigma}$ is the unique element in the set $\mathbb{Z}^{+} \sigma \cap \Phi\left(\Gamma_{X}\right)$. Next, $\bar{\sigma} \in \bar{\Sigma}(G)$ by (\$2). Since $\sigma \in \mathfrak{X}(T)$, an inspection of Table 1 along with Lemma 4.7 shows that the condition $\sigma \notin \bar{\Sigma}(G)$ implies $\bar{\sigma}=2 \sigma$. Hence in what follows we assume $\sigma \in \bar{\Sigma}(G)$. Inspecting again Table 1, we find that $\bar{\sigma}=\sigma$ except for, possibly, one of the following two cases.

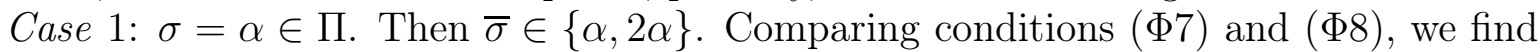
that $\bar{\sigma}=2 \alpha$ if and only if $\mathbb{Q}^{+} \iota\left(\alpha^{\vee}\right)$ is an extremal ray of the cone $\mathcal{K}$.

Case 2: $\sigma=\alpha_{1}+\ldots+\alpha_{r}$ with $\operatorname{Supp} \sigma$ of type $\mathrm{B}_{r}(r \geq 2)$. Then $\bar{\sigma} \in\{\sigma, 2 \sigma\}$. Comparing

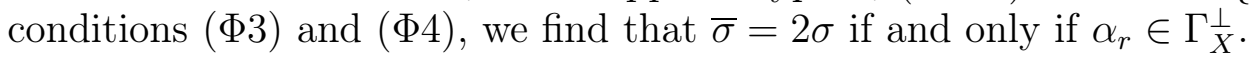


4.3. Consequences for multiplicity-free affine $G$-varieties. In this subsection, using a simple reduction, we extend some of the results of $\S 4.2$ to arbitrary multiplicity-free affine $G$-varieties.

Let $X$ be a multiplicity-free affine $G$-variety and let $\widetilde{X}$ be the normalization of $X$. Clearly, $\widetilde{X}$ is an affine spherical $G$-variety and $\mathbb{k}[X]$ is naturally identified with a $G$ invariant subalgebra of $\mathbb{k}[\widetilde{X}]$.

Proposition 4.20. Up to a G-isomorphism, $X$ is uniquely determined by $\widetilde{X}$ and $\Gamma_{X}$.

Proof. This follows from the fact that $\Gamma_{X}$ uniquely determines $\mathbb{k}[X]$ as a $G$-submodule and hence as a subspace of $\mathbb{k}[\widetilde{X}]$.

Proposition 4.21. The following assertions hold:

(a) $\Gamma_{\tilde{X}}=\mathbb{Z} \Gamma_{X} \cap \mathbb{Q}^{+} \Gamma_{X}$ (that is, $\Gamma_{\widetilde{X}}$ is the saturation of $\left.\Gamma_{X}\right)$;

(b) $\Sigma_{\tilde{X}}=\Sigma_{X}$.

Proof. (国) Since the algebra $\mathbb{k}[\tilde{X}]$ is integral over $\mathbb{k}[X]$, it follows from $[$ Po86, Corollary 2 of Theorem 4] that the algebra $\mathbb{k}[\widetilde{X}]^{U}$ is integral over $\mathbb{k}[X]^{U}$. Taking into account Proposition $\left[2.9\right.$ and the equality $\mathbb{k}(X)^{U}=$ Quot $\mathbb{k}[X]^{U}$ (see [PoV94, Theorem 3.3]), we conclude that $\mathbb{k}[\tilde{X}]^{U}$ is the integral closure of $\mathbb{k}[X]^{U}$ in Quot $\mathbb{k}[X]^{U}$. It remains to apply Proposition 2.7.

(bib) Since $X$ and $\widetilde{X}$ contain the same open $G$-orbit, the claim follows from Proposition B.1 and the definition of the set of spherical roots (see $\$ 2.3$ ).

Corollary 4.22. Up to a G-isomorphism, every multiplicity-free affine $G$-variety $X$ is uniquely determined by the pair $\left(\Gamma_{X}, \Sigma_{X}\right)$.

Proof. Combining Proposition 4.21 and Corollary 4.16, we find that the pair $\left(\Gamma_{X}, \Sigma_{X}\right)$ uniquely determines $\widetilde{X}$ up to a $G$-isomorphism. It remains to apply Proposition 4.20.

The following result was first obtained in [AB05, Corollary 3.4].

Corollary 4.23. Up to a G-isomorphism, there are only finitely many multiplicity-free affine $G$-varieties with a prescribed weight monoid.

Proof. This is implied by Proposition 4.21(国), Corollary 4.17, and Proposition 4.20.

4.4. The uniqueness property for spherical homogeneous spaces. Given a spherical homogeneous space $G / H$, recall its invariants $\Lambda_{G / H}, \Pi_{G / H}^{p}, \Sigma_{G / H}$, and $\mathcal{D}_{G / H}$ from Appendix B. Our goal in this subsection is to give a new proof of the following theorem, which is a reformulation of [Lo09a, Theorem 1].

Theorem 4.24. Up to a $G$-isomorphism, every spherical homogeneous space $G / H$ is uniquely determined by the quadruple $\mathscr{H}_{G / H}=\left(\Lambda_{G / H}, \Pi_{G / H}^{p}, \Sigma_{G / H}, \mathcal{D}_{G / H}\right)$.

The main idea of our proof of this theorem is to perform a reduction to the uniqueness property for affine spherical varieties (Corollary 4.16). The reduction itself uses tools that go back to [Lu01, §6]; see also [Br07, § 3.2].

Recall that a subgroup $H \subset G$ is said to be spherical if $G / H$ is a spherical homogeneous space. In the proof of Theorem 4.24 we shall need the following lemma. 
Lemma 4.25. Suppose that $H$ and $H^{\prime}$ are two spherical subgroups of $G$ such that $H \subset$ $H^{\prime} \subset N_{G}(H)$. Then, modulo the inclusion $\Lambda_{G / H^{\prime}} \hookrightarrow \Lambda_{G / H}$, the equality $\mathbb{Q}^{+} \Sigma_{G / H^{\prime}}=$ $\mathbb{Q}^{+} \Sigma_{G / H}$ holds.

Proof. Restricting valuations along the chain $\mathbb{k}(G / H) \supset \mathbb{k}\left(G / H^{\prime}\right) \supset \mathbb{k}\left(G / N_{G}(H)\right)$ yields a chain of maps

$$
\mathcal{V}_{G / H} \rightarrow \mathcal{V}_{G / H^{\prime}} \rightarrow \mathcal{V}_{G / N_{G}(H)}
$$

As follows from [LuV83, §3.2, Corollary 1] or [Kn91, Corollary 1.5], all the maps in (4.7) are surjective, which induces a chain of inclusions

$$
\mathbb{Q}^{+} \Sigma_{G / N_{G}(H)} \hookrightarrow \mathbb{Q}^{+} \Sigma_{G / H^{\prime}} \hookrightarrow \mathbb{Q}^{+} \Sigma_{G / H}
$$

It was shown in [BP87, §5.4] that the composite map $\mathcal{V}_{G / H} \rightarrow \mathcal{V}_{G / N_{G}(H)}$ in (4.7) is the quotient by the vector subspace $\mathcal{V}_{G / H} \cap\left(-\mathcal{V}_{G / H}\right)$. It follows that the composite map $\mathbb{Q}^{+} \Sigma_{G / N_{G}(H)} \hookrightarrow \mathbb{Q}^{+} \Sigma_{G / H}$ in (4.8) is bijective hence so are all the maps in (4.8).

Proof of Theorem 4.24. Without loss of generality, we may assume that $G$ is the product of a simply connected semisimple group with a torus. Fix a spherical subgroup $H \subset G$.

Let $H^{\sharp} \subset H$ be the common kernel of all characters of $H$. Clearly, $H^{\sharp}$ is a normal subgroup of $H$ and the group $S=H / H^{\sharp}$ is diagonalizable. Consider the natural map

$$
\varphi: H \rightarrow S, \quad h \mapsto h H^{\sharp} .
$$

The definition of $H^{\sharp}$ implies that the induced map $\varphi^{*}: \mathfrak{X}(S) \rightarrow \mathfrak{X}(H)$ is an isomorphism.

Consider the homogeneous space $G / H^{\sharp}$ and equip it with the natural action of the group $G \times S$ given by $\left(\left(g, h H^{\sharp}\right), x H^{\sharp}\right) \mapsto g x h^{-1} H^{\sharp}$. One easily sees that the stabilizer in $G \times S$ of the point $e H^{\sharp}$ is the subgroup

$$
\widehat{H}=\left\{\left(h, h H^{\sharp}\right) \mid h \in H\right\} \simeq H .
$$

In what follows, we identify the algebra $\mathbb{k}\left[G / H^{\sharp}\right]$ with $\mathbb{k}[G]^{H^{\sharp}}$.

The action of $G \times S$ on $G / H^{\sharp}$ induces the $(G \times S)$-module structure on the algebra $\mathbb{k}[G]^{H^{\sharp}}$ given by

$$
\left[\left(g, h H^{\sharp}\right) f\right](x)=f\left(g^{-1} x h\right),
$$

where $g, x \in G, h \in H$, and $f \in \mathbb{k}[G]^{H^{\sharp}}$. It follows from [ViK78, Theorem 1] that the sphericity of $H$ is equivalent to the condition that the $(G \times S)$-module $\mathbb{k}[G]^{H^{\sharp}}$ be multiplicity-free. Let $\widehat{\Gamma}_{G / H}$ be the set of all pairs $(\lambda, \chi) \in \Lambda^{+} \oplus \mathfrak{X}(H)$ such that $\mathbb{k}[G]^{H^{\sharp}}$ contains a simple $(G \times S)$-submodule isomorphic to $V(\lambda) \otimes \mathbb{k}_{\chi}$, where $\mathbb{k}_{\chi}$ stands for the onedimensional $S$-module on which $S$ acts via the character $\chi$. The set $\widehat{\Gamma}_{G / H}$ is a submonoid in $\Lambda^{+} \oplus \mathfrak{X}(H)$, called the extended weight monoid of $G / H$; see [Av15, §2.2] for details.

The variety $G / H^{\sharp}$ is quasi-affine (see, for instance, [Av15, Lemma 2.4]). It is thus identified with an open $(G \times S)$-stable subset of the affine $(G \times S)$-variety $X=\operatorname{Spec} \mathbb{k}[G]^{H^{\sharp}}$. By the definitions of $\widehat{\Gamma}_{G / H}$ and $X$, there is a $(G \times S)$-module isomorphism

$$
\mathbb{k}[X] \simeq \bigoplus_{(\lambda, \chi) \in \widehat{\Gamma}_{G / H}} V(\lambda) \otimes \mathbb{k}_{\chi} .
$$

Now consider the subgroup $H^{0} \subset G$, which is also spherical. Lemma 4.25 yields

$$
\mathbb{Q}^{+} \Sigma_{G / H}=\mathbb{Q}^{+} \Sigma_{G / H^{0}} .
$$


By [BP87, Corollary 5.2], the group $N_{G}\left(H^{0}\right) / H^{0}$ is diagonalizable, hence so is $H / H^{0}$. It follows that

$$
H^{0}=\varphi^{-1}\left(S^{0}\right)
$$

where the map $\varphi$ is given by (4.9).

Observe that the group $G \times S^{0}$ acts transitively on $G / H^{\sharp}$ and that the stabilizer in $G \times S^{0}$ of the point $e H^{\sharp}$ is the subgroup $\widehat{H}^{0}$. It follows from the sphericity of $H^{0}$ that $G / H^{\sharp}$ is a spherical $\left(G \times S^{0}\right)$-variety, hence so is $X$. In particular, the algebra $\mathbb{k}[X]$ is a multiplicity-free $\left(G \times S^{0}\right)$-module. Consequently, the natural map

$$
\psi: \widehat{\Gamma}_{G / H} \rightarrow \Gamma_{X}, \quad(\lambda, \chi) \mapsto\left(\lambda,\left.\chi\right|_{S^{0}}\right),
$$

is injective and hence an isomorphism.

It is easy to see that $\widehat{H}^{0} \subset H^{0} \times S^{0} \subset N_{G \times S^{0}}\left(\widehat{H}^{0}\right)$ and $\left(G \times S^{0}\right) /\left(H^{0} \times S^{0}\right) \simeq G / H^{0}$. This together with Lemma 4.25 implies $\mathbb{Q}^{+} \Sigma_{G / H^{0}}=\mathbb{Q}^{+} \Sigma_{\left(G \times S^{0}\right) / \widehat{H}^{0}}$. Combining this equality with (4.11) yields

$$
\mathbb{Q}^{+} \Sigma_{G / H}=\mathbb{Q}^{+} \Sigma_{X}
$$

We are now ready to recover $H$ from $\mathscr{H}_{G / H}$. As shown in [Av15, § 2.3], the datum $\mathscr{H}_{G / H}$ uniquely determines $\mathfrak{X}(H)$ as an abstract group and $\widehat{\Gamma}_{G / H}$ as a submonoid of $\Lambda^{+} \oplus \mathfrak{X}(H)$. Then $S$ is recovered as the diagonalizable group with $\mathfrak{X}(S)=\mathfrak{X}(H)$. Next, the weight monoid $\Gamma_{X}$ is recovered by the formula $\Gamma_{X}=\psi\left(\widehat{\Gamma}_{G / H}\right)$. Further, equality (4.14) together with $\Gamma_{X}$ uniquely determine the set $\Sigma_{X}$. According to Corollary 4.16, the pair $\left(\Gamma_{X}, \Sigma_{X}\right)$ uniquely determines $X$ up to a $\left(G \times S^{0}\right)$-isomorphism. As the map $\psi$ is injective, the action of $G \times S^{0}$ on $X$ uniquely extends to an action of $G \times S$ satisfying (4.10). Therefore $X$ is uniquely determined up to $(G \times S)$-equivariant isomorphism. At last, up to conjugacy, the subgroup $H$ is recovered from $X$ as the projection to $G$ of the stabilizer in $G \times S$ of a point in the open $(G \times S)$-orbit in $X$.

\section{Appendix A. The structure constants of Chevalley Bases}

Computations carried out in $\$ 3.8$ require the knowledge of signs of the structure constants of Chevalley bases for the simple Lie algebras of types $\mathrm{A}_{r}, \mathrm{~B}_{r}, \mathrm{C}_{r}, \mathrm{D}_{r}, \mathrm{~F}_{4}$, and $\mathrm{G}_{2}$. The goal of this appendix is to specify a particular choice of the signs for each of the above-mentioned Lie algebras.

Let $\mathfrak{g}$ be a simple Lie algebra and let $\left\{h_{\alpha} \mid \alpha \in \Pi\right\} \cup\left\{e_{\alpha} \mid \alpha \in \Delta\right\}$ be a Chevalley basis of $\mathfrak{g}$. The following relations for $\alpha, \beta \in \Delta^{+}$easily follow, for instance, from Ca89, Theorem 4.1.2]:

$$
\begin{gathered}
N_{\alpha, \beta}=-N_{\beta, \alpha} ; \\
N_{-\alpha,-\beta}=-N_{\alpha, \beta} ; \\
N_{\alpha,-\beta}= \begin{cases}-N_{\beta, \alpha-\beta} \frac{(\alpha-\beta, \alpha-\beta)}{(\alpha, \alpha)} & \text { if } \alpha-\beta \in \Delta^{+} ; \\
N_{\beta-\alpha, \alpha} \frac{(\beta-\alpha, \beta-\alpha)}{(\beta, \beta)} & \text { if } \beta-\alpha \in \Delta^{+} .\end{cases}
\end{gathered}
$$

These relations show that the signs of all the structure constants of $\mathfrak{g}$ are uniquely determined by the signs of the structure constants $N_{\alpha, \beta}$ with $\alpha, \beta \in \Delta^{+}$. In what follows we specify these signs for all the Lie algebras in question.

For type $\mathrm{F}_{4}$, we use the signs presented in [VaP96, Table II]. 
For type $\mathrm{G}_{2}$, we use the signs extracted from [VaP96, Table IV].

For each of the types $\mathrm{A}_{r}, \mathrm{~B}_{r}, \mathrm{C}_{r}, \mathrm{D}_{r}$, a specific choice of the signs is presented below. It can be obtained from explicit matrix realizations of the corresponding simple Lie algebras.

Type $\mathrm{A}_{r}, r \geq 2$.

For $1 \leq i \leq j \leq r$ set $\alpha_{i j}=\alpha_{i}+\ldots+\alpha_{j}$. Then $\Delta^{+}=\left\{\alpha_{i j} \mid 1 \leq i \leq j \leq r\right\}$.

\begin{tabular}{|c|c|c|}
\hline Condition & $k=j+1$ & $i=l+1$ \\
\hline Sign of $N_{\alpha_{i j}, \alpha_{k l}}$ & + & - \\
\hline
\end{tabular}

Type $\mathrm{B}_{r}, r \geq 2$.

For $1 \leq i \leq j \leq r$ set $\alpha_{i j}=\alpha_{i}+\ldots+\alpha_{j}$.

For $1 \leq i<j \leq r$ set $\beta_{i j}=\alpha_{i r}+\alpha_{j r}$.

Then $\Delta^{+}=\left\{\alpha_{i j} \mid 1 \leq i \leq j \leq r\right\} \cup\left\{\beta_{i j} \mid 1 \leq i<j \leq r\right\}$.

\begin{tabular}{|c|c|c|c|c|}
\hline Condition & $k=j+1$ & $i=l+1$ & $j=l=r, i<k$ & $j=l=r, k<i$ \\
\hline Sign of $N_{\alpha_{i j}, \alpha_{k l}}$ & + & - & - & + \\
\hline
\end{tabular}

\begin{tabular}{|c|c|c|c|}
\hline Condition & $i=l+1$ & $j=l+1, k<i$ & $j=l+1, i<k$ \\
\hline Sign of $N_{\beta_{i j}, \alpha_{k l}}$ & - & + & - \\
\hline
\end{tabular}

Type $\mathrm{C}_{r}, r \geq 3$.

For $1 \leq i \leq j \leq r-1$ set $\alpha_{i j}=\alpha_{i}+\ldots+\alpha_{j}$.

For $1 \leq i \leq r$ set $\beta_{i r}=\alpha_{i}+\ldots+\alpha_{r}$.

For $1 \leq i \leq j<r$ set $\beta_{i j}=\alpha_{i, r-1}+\alpha_{r}+\alpha_{j, r-1}$.

Then $\Delta^{+}=\left\{\alpha_{i j} \mid 1 \leq i \leq j \leq r-1\right\} \cup\left\{\beta_{i j} \mid 1 \leq i \leq j \leq r\right\}$.

\begin{tabular}{|c|c|c|}
\hline Condition & $k=j+1$ & $i=l+1$ \\
\hline Sign of $N_{\alpha_{i j}, \alpha_{k l}}$ & + & - \\
\hline \hline Condition & $i=l+1$ & $j=l+1$ \\
\hline Sign of $N_{\beta_{i j}, \alpha_{k l}}$ & - & - \\
\hline
\end{tabular}

Type $\mathrm{D}_{r}, r \geq 4$.

For $1 \leq i \leq j \leq r-1$ set $\alpha_{i j}=\alpha_{i}+\ldots+\alpha_{j}$.

For $1 \leq i \leq r-1$ set $\beta_{i r}=\alpha_{i, r-1}+\left(\alpha_{r}-\alpha_{r-1}\right)$.

For $1 \leq i<j \leq r-1$ set $\beta_{i j}=\alpha_{i, r-1}+\left(\alpha_{r}-\alpha_{r-1}\right)+\alpha_{j, r-1}$.

Then $\Delta^{+}=\left\{\alpha_{i j} \mid 1 \leq i \leq j \leq r-1\right\} \cup\left\{\beta_{i j} \mid 1 \leq i<j \leq r\right\}$.

\begin{tabular}{|c|c|c|}
\hline Condition & $k=j+1$ & $i=l+1$ \\
\hline Sign of $N_{\alpha_{i j}, \alpha_{k l}}$ & + & - \\
\hline
\end{tabular}

\begin{tabular}{|c|c|c|c|}
\hline Condition & $i=l+1$ & $j=l+1, k<i$ & $j=l+1, i<k$ \\
\hline Sign of $N_{\beta_{i j}, \alpha_{k l}}$ & - & + & - \\
\hline
\end{tabular}

APPENDix B. InVARIANTS OF SPHERICAL HOMOGENEOUS SPACES

In this appendix we recall combinatorial invariants of spherical homogeneous spaces and their equivariant embeddings used in this paper. In what follows, $G / H$ is an arbitrary spherical homogeneous space.

Let $P$ denote the stabilizer of the open $B$-orbit in $G / H$. Clearly, $P$ is a parabolic subgroup of $G$ containing $B$. We set

$$
\Pi_{G / H}^{p}=\left\{\alpha \in \Pi \mid e_{-\alpha} \in \mathfrak{p}\right\} .
$$


The next invariants of $G / H$ are the weight lattice

$$
\Lambda_{G / H}=\left\{\lambda \in \mathfrak{X}(T) \mid \mathbb{k}(G / H)_{\lambda}^{(B)} \neq\{0\}\right\}
$$

and the corresponding dual $\mathbb{Q}$-vector space

$$
\mathcal{Q}_{G / H}=\operatorname{Hom}_{\mathbb{Z}}\left(\Lambda_{G / H}, \mathbb{Q}\right) .
$$

For every $\lambda \in \Lambda_{G / H}$, we fix a nonzero rational function $f_{\lambda} \in \mathbb{k}(G / H)_{\lambda}^{(B)}$. Since $G / H$ contains an open $B$-orbit, it follows that $\mathbb{k}(G / H)_{\lambda}^{(B)}=\mathbb{k} f_{\lambda}$ for all $\lambda \in \Lambda_{G / H}$.

Every discrete $\mathbb{Q}$-valued valuation $v$ of $\mathbb{k}(G / H)$ vanishing on $\mathbb{k}^{\times}$determines an element $\rho_{v} \in \mathcal{Q}_{G / H}$ such that $\left\langle\rho_{v}, \lambda\right\rangle=v\left(f_{\lambda}\right)$ for all $\lambda \in \Lambda_{G / H}$. It is known (see [LuV83, 7.4] or [Kn91, Corollary 1.8]) that the restriction of the map $v \mapsto \rho_{v}$ to the set of $G$-invariant $\mathbb{Q}$-valued valuations of $\mathbb{k}(G / H)$ vanishing on $\mathbb{k}^{\times}$is injective; we denote its image by $\mathcal{V}_{G / H}$. It was proved in [Br90, $\S 3]$ that $\mathcal{V}_{G / H}$ is a cosimplicial cone in $\mathcal{Q}_{G / H}$. Consequently, there is a uniquely determined linearly independent set $\Sigma_{G / H}$ of primitive elements in $\Lambda_{G / H}$ such that

$$
\mathcal{V}_{G / H}=\left\{q \in \mathcal{Q}_{G / H} \mid\langle q, \sigma\rangle \leq 0 \text { for all } \sigma \in \Sigma_{G / H}\right\} .
$$

Elements of $\Sigma_{G / H}$ are called spherical roots of $G / H$ and $\mathcal{V}_{G / H}$ is called the valuation cone of $G / H$.

Let $\mathcal{D}_{G / H}$ denote the set of $B$-stable prime divisors in $G / H$. Elements of $\mathcal{D}_{G / H}$ are called colors of $G / H$. For every $D \in \mathcal{D}_{G / H}$, let $v_{D}$ be the valuation of $\mathbb{k}(G / H)$ defined by $D$, that is, $v_{D}(f)=\operatorname{ord}_{D}(f)$ for every $f \in \mathbb{k}(G / H)$. Let $\rho_{G / H}: \mathcal{D}_{G / H} \rightarrow \mathcal{Q}_{G / H}$ be the map given by $\rho_{G / H}(D)=\rho_{v_{D}}$ for all $D \in \mathcal{D}_{G / H}$. We regard $\mathcal{D}_{G / H}$ as an abstract set equipped with the map $\rho_{G / H}$.

For an arbitrary irreducible $G$-variety $X$ containing $G / H$ as an open $G$-orbit, one defines the same invariants $\Pi_{X}^{p}, \Lambda_{X}, \mathcal{Q}_{X}, \mathcal{V}_{X}, \Sigma_{X}, \mathcal{D}_{X}$, and $\rho_{X}$ of $X$ as those of $G / H$.

For a multiplicity-free affine $G$-variety $X$, the set $\Sigma_{X}$ defined right above coincides with the set $\Sigma_{X}$ defined in $\S 2.3$. This follows from the following proposition, which is a particular case of [Kn96, Lemma 6.6, iii)].

Proposition B.1. Suppose that $X$ is a multiplicity-free affine G-variety. Then the cone $\mathbb{Q}^{+} \Xi_{X}$ is dual to $-\mathcal{V}_{X}$.

\section{REFERENCES}

[AB05] V. Alexeev, M. Brion, Moduli of affine schemes with reductive group action, J. Algebraic Geom. 14 (2005), no. 1, 83-117; see also arXiv:math/0301288 [math.AG].

[Av15] R. Avdeev, Strongly solvable spherical subgroups and their combinatorial invariants, Selecta Math. (N.S.) 21 (2015), no. 3, 931-993; see also arXiv:1212.3256 [math.AG].

[ACF18] R. Avdeev, S. Cupit-Foutou, On the irreducible components of moduli schemes for affine spherical varieties, Transform. Groups 23 (2018), to appear, DOI:10.1007/s00031-017-9443-8; see also arXiv: 1406.1713v4 [math.AG].

[Bo68] N. Bourbaki, Éléments de mathématique. Groupes et Algèbres de Lie. Chapitre IV: Groupes de Coxeter et Systèmes de Tits. Chapitre V: Groupes engendrés par des réflexions. Chapitre VI: Systèmes de racines. Actualités Scientifiques et Industrielles, No. 1337 Hermann, Paris, 1968.

[BCF08] P. Bravi, S. Cupit-Foutou, Equivariant deformations of the affine multicone over a flag variety, Adv. Math. 217 (2008), no. 6, 2800-2821; see also arXiv:math/0603690 [math. AG]. 
[BVS16] P. Bravi, B. Van Steirteghem, The moduli scheme of affine spherical varieties with a free weight monoid, Int. Math. Res. Not. IMRN 2016 (2016), no. 15, 4544-4587; see also arXiv:1406.6041 [math.AG].

[Br90] M. Brion, Vers une généralisation des espaces symétriques, J. Algebra 134 (1990), no. 1, $115-143$.

[Br07] M. Brion, The total coordinate ring of a wonderful variety, J. Algebra 313 (2007), 61-99.

[Br13] M. Brion, Invariant Hilbert schemes, Handbook of Moduli, Vol. I, Adv. Lect. in Math. 24, 63-118, International Press, 2013; see also arXiv:1102.0198 [math.AG].

[BP87] M. Brion, F. Pauer, Valuations des espaces homogènes sphériques, Comment. Math. Helv. 62 (1987), no. 2, 265-285.

[Ca89] R. W. Carter, Simple groups of Lie type, Wiley Classics Library, John Wiley \& Sons, 1989.

[CF09] S. Cupit-Foutou, Wonderful varieties: a geometrical realization, preprint, see arXiv:0907.2852 [math.AG].

[Ha67] D. Hadziev, Some questions in the theory of vector invariants, Math. USSR-Sb. 1 (1967), no. $3,383-396$.

[Ha77] R. Hartshorne, Algebraic geometry, Graduate texts in mathematics, no. 52, Springer, New York Heidelberg, 1977.

[Ja07] S. Jansou, Déformations des cônes de vecteurs primitifs, Math. Ann. 338 (2007), no. 3, 627667; see also arXiv:math/0506133 [math.AG].

[KKMS73] G. Kempf, F. Knudsen, D. Mumford, B. Saint-Donat, Toroidal embeddings I, Lecture Notes in Mathematics, vol. 339, Springer, Berlin-New York, 1973.

[Kn91] F. Knop, The Luna-Vust theory of spherical embeddings, Proceedings of the Hyderabad Conference on Algebraic Groups (Hyderabad, India, 1989), Manoj Prakashan, Madras, 1991, $225-249$.

[Kn94] F. Knop, A Harish-Chandra homomorphism for reductive group actions, Ann. of Math. (2) 140 (1994), no. 2, 253-288.

[Kn96] F. Knop, Automorphisms, root systems, and compactifications of homogeneous varieties, J. Amer. Math. Soc. 9 (1996), no. 1, 153-174.

[Li02] Q. Liu, Algebraic geometry and arithmetic curves, Oxford graduate texts in mathematics, 6 , Oxford University Press, Oxford, 2002.

[Lo09a] I. Losev, Uniqueness property for spherical homogeneous spaces, Duke Math. J. 147 (2009), no. 2, 315-343; see also arXiv:math/0703543 [math.AG].

[Lo09b] I. V. Losev, Proof of the Knop conjecture, Ann. Inst. Fourier 59 (2009), no. 3, 1105-1134; see also arXiv:math/0612561 [math.AG].

[Lu01] D. Luna, Variétés sphériques de type A, Inst. Hautes Études Sci. Publ. Math. 94 (2001), 161-226.

[LuV83] D. Luna, Th. Vust, Plongements d'espaces homogènes, Comment. Math. Helv. 58 (1983), no. 2, 186-245.

[PVS12] S. A. Papadakis, B. Van Steirteghem, Equivariant degenerations of spherical modules for groups of type A, Ann. Inst. Fourier 62 (2012), no. 5, 1765-1809; see also arXiv:1008.0911 [math.AG].

[PVS16] S.A. Papadakis, B. Van Steirteghem, Equivariant degenerations of spherical modules: part II, Algebr. Represent. Theory 19 (2016), no. 5, 1135-1171; see also arXiv: 1505.07446 [math.AG]

[Po86] V.L. Popov, Contraction of the actions of reductive algebraic groups, Math. USSR-Sb. 58 (1987), no. 2, 311-335.

[PoV94] V. L. Popov, E. B. Vinberg, Invariant theory, Algebraic geometry. IV: Linear algebraic groups, invariant theory, Encycl. Math. Sci., vol. 55, 1994, pp. 123-278.

[Ti11] D. A. Timashev, Homogeneous spaces and equivariant embeddings, Encycl. Math. Sci., vol. 138, Springer, Berlin Heidelberg, 2011.

[VaP96] N. Vavilov, E. Plotkin, Chevalley groups over commutative rings: I. Elementary calculations, Acta Appl. Math. 45 (1996), 73-113. 
[ViK78] E. B. Vinberg, B. N. Kimel'fel'd, Homogeneous domains on flag manifolds and spherical subgroups of semisimple Lie groups, Funct. Anal. Appl. 12 (1978), no. 3, 168-174.

[ViP72] E. B. Vinberg, V. L. Popov, On a class of quasihomogeneous affine varieties, Math. USSR-Izv. 6 (1972), no. 4, 743-758.

[Vu76] Th. Vust, Sur la théorie des invariants des groupes classiques, Ann. Inst. Fourier (Grenoble) 26 (1976), no. 1, 1-31.

\section{Roman Avdeev}

National Research University "Higher School of Economics", Moscow, Russia

E-mail address: suselr@yandex.ru

\section{Stéphanie Cupit-Foutou}

Ruhr-Universität Bochum, NA 4/67, D-44797 Bochum, Germany

E-mail address: stephanie.cupit@rub.de 